\title{
Development of a multiplexed RNAi-coupled sensor assay to study neuronal function on the large-scale
}

\author{
Dissertation \\ for the award of the degree \\ "Doctor rerum naturalium" \\ of the Georg-August-University Göttingen \\ within the doctoral program \\ GGNB Molecular Physiology of the Brain \\ of the Georg-August University School of Science (GAUSS)
}

submitted by

Alexander Herholt

from Bielefeld, Germany

Göttingen, 2016 
Thesis Committee:

\section{Prof. Dr. Moritz Rossner}

Molecular Neurobiology, Department of Psychiatry, LMU München

\section{Prof. Dr. Nils Brose}

Department of Molecular Neurobiology, MPI of Experimental Medicine Göttingen

\section{Dr. Camin Dean}

Trans-synaptic Signaling, European Neuroscience Institute Göttingen

Members of the Examination Board:

\section{Prof. Dr. Moritz Rossner (Referee)}

Molecular Neurobiology, Department of Psychiatry, LMU München

\section{Prof. Dr. Nils Brose (2 ${ }^{\text {nd }}$ Referee)}

Department of Molecular Neurobiology, MPI of Experimental Medicine Göttingen

\section{Dr. Camin Dean}

Trans-synaptic Signaling, European Neuroscience Institute Göttingen

Further members of the Examination Board:

\section{Prof. Dr. Martin Göpfert}

Department of Cellular Neurobiology, Georg-August-University Göttingen

\section{Prof. Dr. Mikael Simons}

Cellular Neuroscience, MPI of Experimental Medicine Göttingen Institute of Neuronal Cell Biology, DZNE, München

\section{Prof. Dr. Michael Sereda}

Molecular and Translational Neurology, Department of Neurogenetics, MPI of Experimental Medicine Göttingen 


\section{Declaration}

Herewith I declare that I prepared the $\mathrm{PhD}$ thesis entitled: 'Development of a multiplexed RNAi-coupled sensor assay to study neuronal function on the large-scale' on my own and with no other sources and aids than quoted.

Alexander Herholt

$31^{\text {st }}$ Mai, 2016

Göttingen 


\section{Contents}

Contents

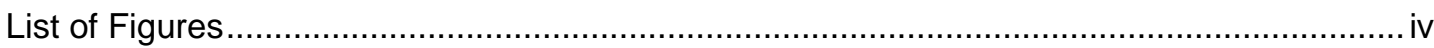

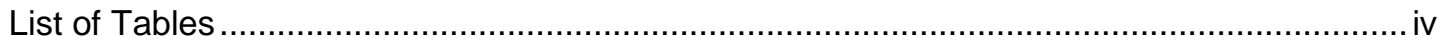

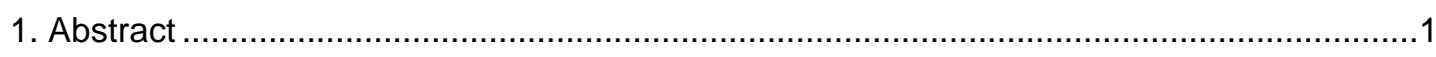

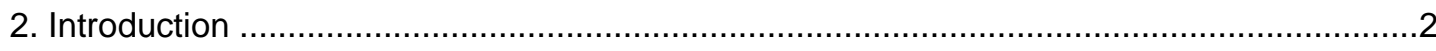

2.1. Synaptic plasticity and excitation-transcription coupling in higher brain function ......... 2

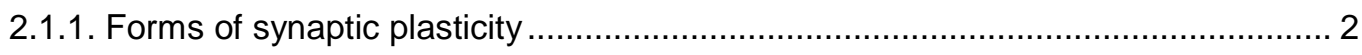

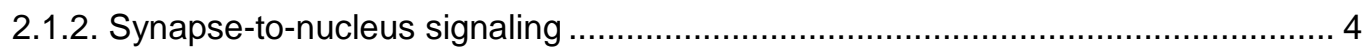

2.2. Synaptic dysfunction as a converging point of psychiatric diseases .......................... 8

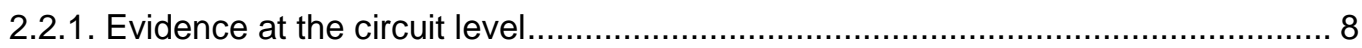

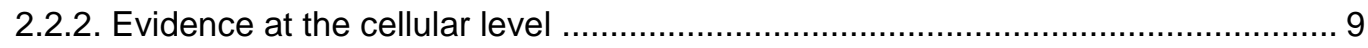

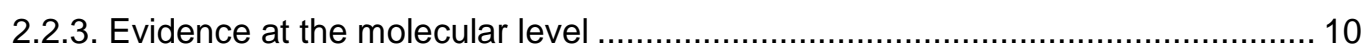

2.3. Inventory of the high-throughput toolbox in neuroscience ...................................... 13

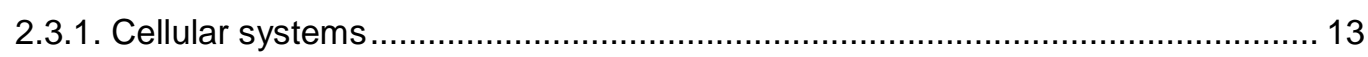

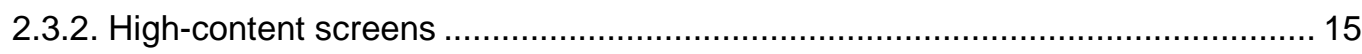

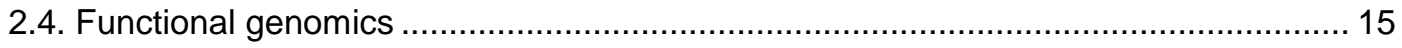

2.4.1. Genetic perturbation by RNA interference and CRISPR-Cas9......................... 16

2.4.2. Advances through molecular barcoding and its application............................... 20

2.4.3. Pooled RNAi/CRISPR-Cas9 screenings ....................................................... 21

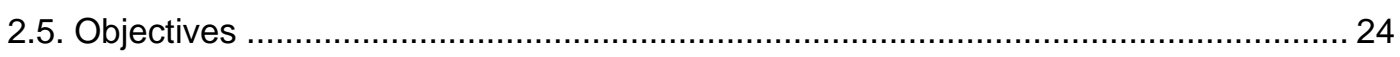

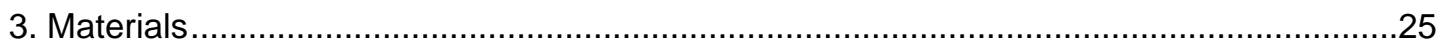

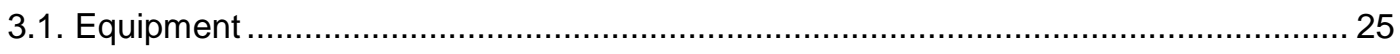

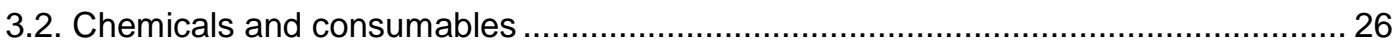

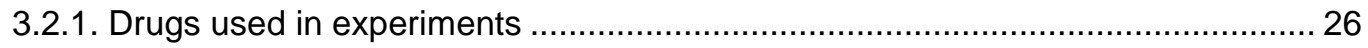

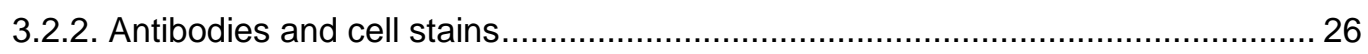

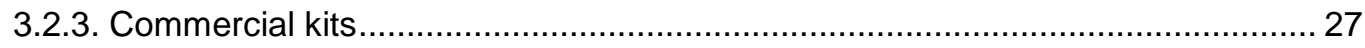

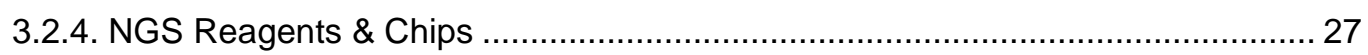

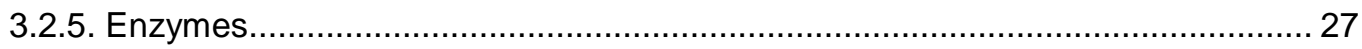

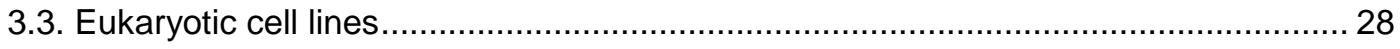


3.4. Bacterial strains

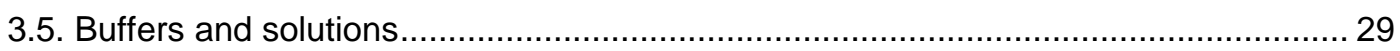

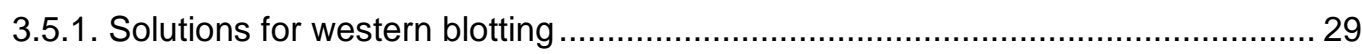

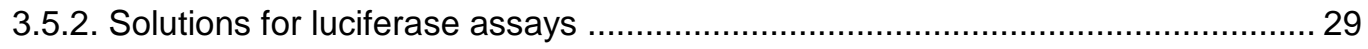

3.5.3. Solutions and media for cell culture .......................................................... 30

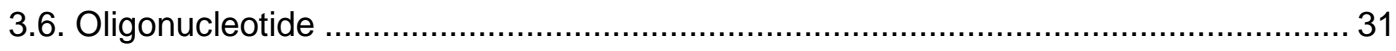

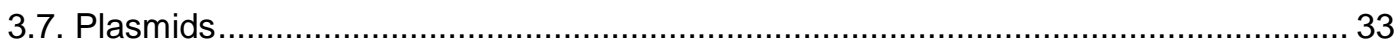

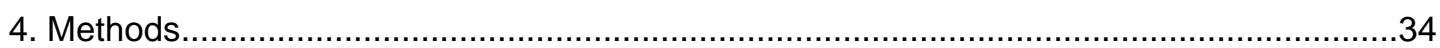

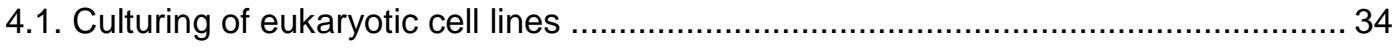

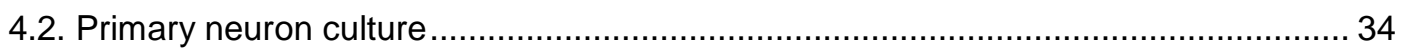

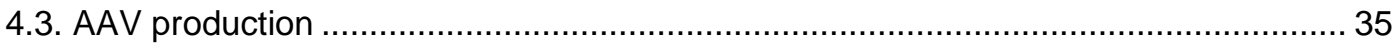

4.4. Rotenone dose-response cell viability assay ........................................................ 37

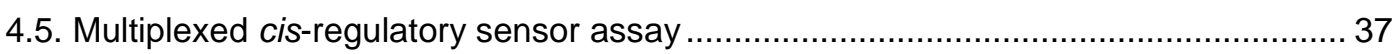

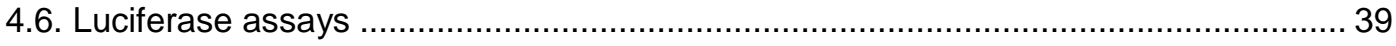

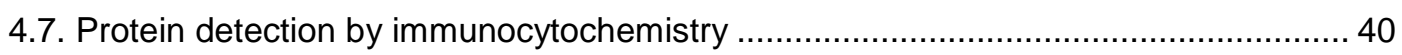

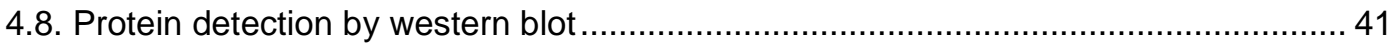

4.9. RNA detection by reverse transcription and qRT-PCR ......................................... 41

4.10. Design and cloning of the PATHscreener library ................................................. 42

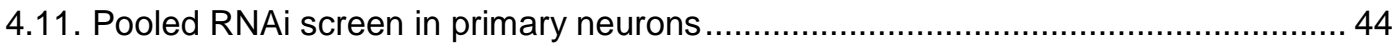

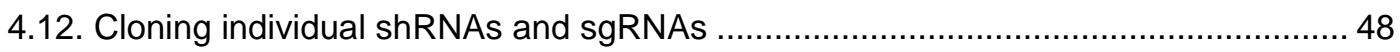

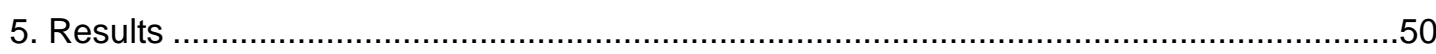

5.1. General considerations for a pooled RNAi screen in neurons ................................. 50

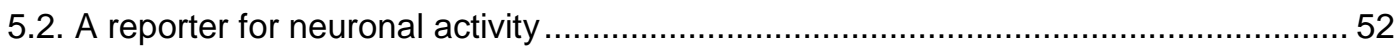

5.3. Vector design for sensor-based RNAi screenings ............................................... 56

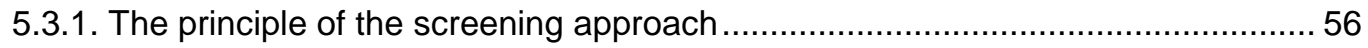

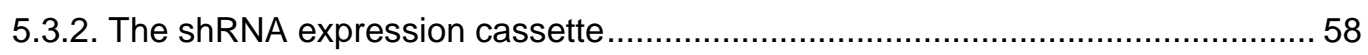

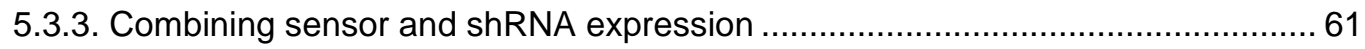

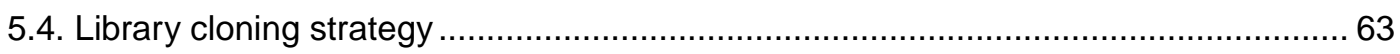

5.5. Sensor-based pooled RNAi screen in primary neurons.......................................... 65

5.5.1. Proof-of-concept screen for regulators of neuronal excitation ...........................65 65 
5.5.2. Quality controls within the screening pipeline

5.5.3. Hit nomination by enhanced Z-score ranking and DESeq2 analysis..................67 67

5.5.4. Hit ranking to biological function by KEGG and Reactome analyses .................. 70

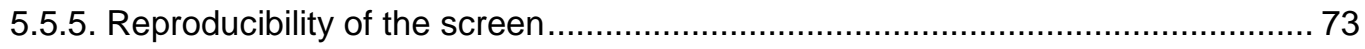

5.5.6. Screening for negative regulators of neuronal excitation................................. 75

5.6. Adaptation towards CRISPR-Cas9-based screening in primary neurons .................. 76

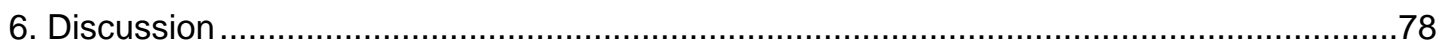

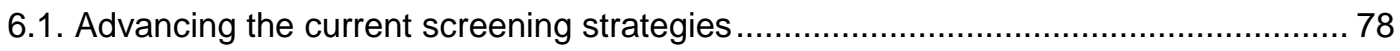

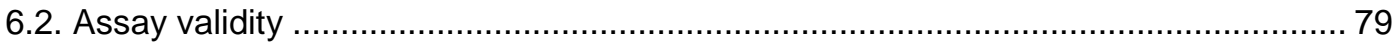

6.2.1. Identification of genes with known function in synaptic plasticity ....................... 79

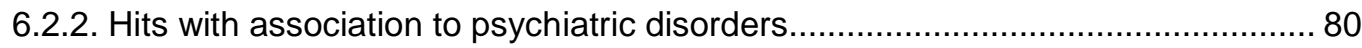

6.2.3. Chemokine signaling: Growing evidence for its role in neuronal activity ............. 81

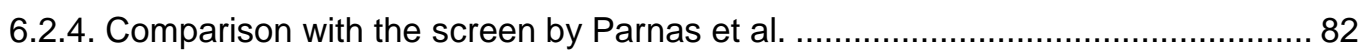

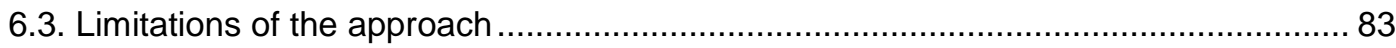

6.4. Future aspects of pooled genetic screens in neuroscience ...................................... 83

6.4.1. Importance for psychiatric drug discovery .................................................. 84

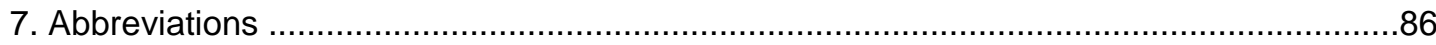

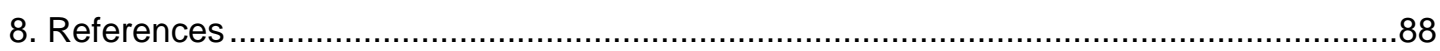

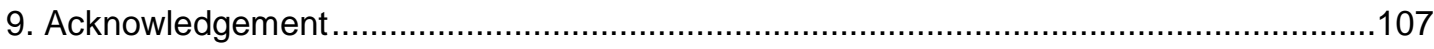

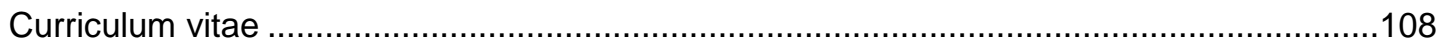




\section{List of Figures}

Figure 1: Regulatory pathways in synaptic plasticity and activity-dependent gene expression.

Figure 2: Alteration in cortical circuit function in schizophrenia. ......................................... 9

Figure 3: Summary of genetic associations for MDD, BPD, ASD, and SZD. ....................... 12

Figure 4: Maturation stages of murine primary neurons. ................................................... 14

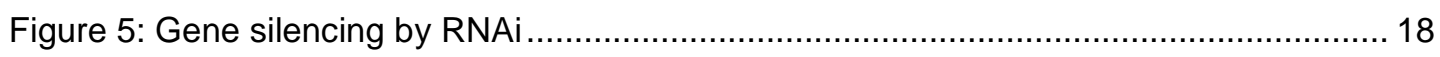

Figure 6: Overview of CRISPR-Cas9 applications........................................................ 20

Figure 7: Current paradigms for pooled genetic screens............................................... 23

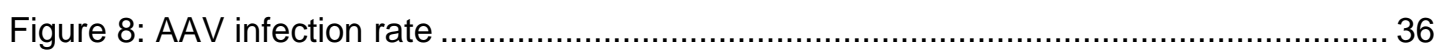

Figure 9: Cellular RNA content is a more sensitive indicator of neuronal viability in the dish

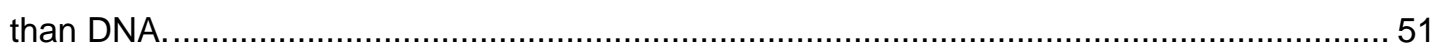

Figure 10: Multiplexed cis-regulatory sensor assay in response to neuronal silencing and

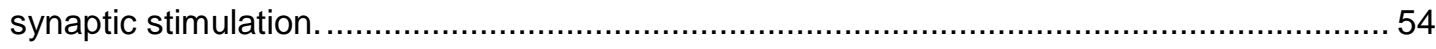

Figure 11: Genomic architecture of the murine Arc promoter.............................................. 55

Figure 12: Design and characterization of the artificial E-SARE sensor. ............................. 56

Figure 13: Principle of the sensor-based genetic interference screen. .............................. 58

Figure 14: Efficient mRNA knockdown by hU6 promoter-driven shRNAs in neuronal cells. . 60

Figure 15: Functional validation of the PATHscreener vector. ............................................ 62

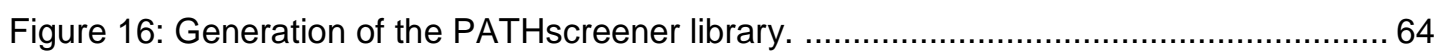

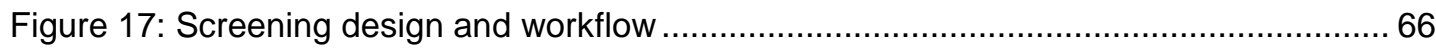

Figure 18: Quality control measures from the pooled RNAi screen...................................... 67

Figure 19: Enhanced Z-score and DESeq2 analysis of the pooled RNAi screen A. ............69

Figure 20: Individual validation of selected candidates. ................................................. 70

Figure 21: Screening hits along the synapse-to-nucleus pathway. .................................... 73

Figure 22: Reproducibility of the pooled RNAi screen in primary neurons. ........................... 74

Figure 23: Comparison of identified negative regulators in screen A, B, and C. .................. 75

Figure 24: Adaptation towards an AAV CRISPR screening vector. ...................................... 77

\section{List of Tables}

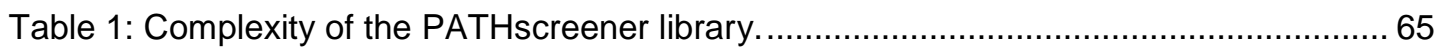

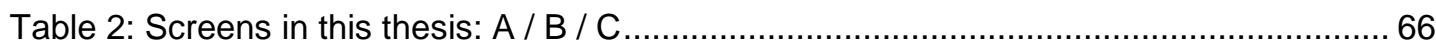

Table 3: KEGG Pathway analysis for the Top140 positive regulators ................................. 71

Table 4: Reactome Pathway analysis for the Top140 positive regulators ............................. 72 


\section{Abstract}

Psychiatric diseases such as schizophrenia, bipolar disorder and autism spectrum disorders are considered neurodevelopmental synaptopathies. Compelling evidence obtained from large-scale genome-wide association studies, identified a plethora of genetic variations within hundreds of genes encoding components of the post-synaptic compartment and calcium signaling mediating excitation-transcription-coupling. This synapse-to-nucleus signaling is thought to be particularly important for synaptic plasticity and learning and memory. In the last decade, tremendous progress has been made in neuroscience research when employing an array of molecular and cellular techniques to study the impact of individual genes on synaptic plasticity. Nevertheless, neuroscience methodology lags behind the field of cancer research in terms of large scale functional genetic screens, e.g. mediated by RNA interference (RNAi). The underlying cause is likely due to both the difficulties of culturing post-mitotic neurons and the phenotypic complexity. In this regard, comprehensively identifying genes involved in neuronal excitation and synapse-to-nucleus signaling may not only deepen our understanding of the corresponding biological processes, but might also be key in unearthing promising targets for psychiatric drug discovery.

I have developed a functional genomics tool that is applicable to primary neurons and combines the throughput of a pooled RNAi screen with the sensitivity of a pathway reporter assay based on the synaptic activity-response element, modified from the Arc enhancer. This thesis describes a proof-of-concept study in which an AAV-based RNAi library was screened for regulators of neuronal excitation and synapse-to-nucleus signaling. The assay principle relies on molecular barcodes, which serve as quantitative reporters, while at the same time also functioning as unique identifiers of the targeted genes. Upon synaptic stimulation, the screen identified a multitude of known genes involved in glutamatergic synapse-to-nucleus signaling, as well as previously unknown candidates like the chemokine receptor XCR1. The technical approach's reproducibility has been verified by substantial overlap of gene hits during three independent screens. Later in the thesis, I also present the principal applicability of CRISPR-Cas9 tools in neurons, which may improve performance for genetic interference screens in the near future.

This assay seeks to enhance the analytic toolbox used for analyzing regulatory processes during neuronal signaling and for the identification of novel targets in psychiatric drug discovery. 


\section{Introduction}

\subsection{Synaptic plasticity and excitation-transcription coupling in higher brain function}

The human brain contains over 80 billion neurons (Azevedo et al., 2009). Those neurons are connected with each other via thousands of synaptic connections in order to transmit electrical signals for communication (Williams and Herrup, 1988). During brain development neurons of various types organize into distinct brain regions (e.g. cortex and hippocampus) and form local circuits (Hensch, 2005). Spatially separated circuits are connected by hubs of neurons that mediate long-range communication (Bullmore and Sporns, 2009). Although the exact mechanisms by which information is stored and processed in the brain is still unknown, neuronal plasticity is key to higher brain function during learning and memory (Amtul and Atta-Ur-Rahman, 2015). This involves strengthening of synaptic contacts, their maintenance as well as their weakening. Neuronal plasticity thereby modulates the intrinsic excitability of neurons and the strength of their synaptic connections. The persistence of such modifications at the cellular level depends on whether the intensity and duration of activity triggers a transcriptional response and the expression of new synaptic proteins (Kandel, 2001). The molecular mechanisms of this excitation-transcription coupling are intensively studied and key players have been identified (Bading, 2013; Greer and Greenberg, 2008; West et al., 2002). Emphasizing the importance of neuronal plasticity for higher brain function, it is not surprising that many neuropsychiatric diseases are associated with synaptic dysfunction (West and Greenberg, 2011).

\subsubsection{Forms of synaptic plasticity}

Synaptic plasticity integrates various kinds of responses to activity leading to changes in synaptic strength and efficacy. Such modifications play a key role during experience-driven information-processing and -storage in the brain. In general, one can categorize the synaptic modifications into forms of short-term and long-term synaptic plasticity (Citri and Malenka, 2008).

Although, the presented study mainly deals with the molecular program leading to longlasting synaptic changes, concepts of short-term plasticity will be introduced briefly for the sake of completeness. Short-term plasticity covers changes that persist for milliseconds to several minutes and they mainly involve modifications of the neurotransmitter release probability at the presynaptic terminal (Thomson, 2000). The release probability can be either facilitated or depressed as a consequence of trains of stimuli and this seems to depend on the frequency of stimuli and the recent history of stimulation at the synapse 
(Zucker and Regehr, 2002). Stimulation in short intervals of less than 20 ms commonly elicit a reduced response to the second stimuli compared to the first one, a phenomenon called paired-pulse depression. The counterpart, paired-pulse facilitation can occur at longer intervals of 20-500 ms. Furthermore, facilitation is rather observed if the synapse had a low neurotransmitter release probability prior to the stimulus and vice versa for depression (Dobrunz and Stevens, 1997). Regulators of the release property are, for example, presynaptic ionotropic receptors, such as the kainate receptor and the nicotinic acetylcholine receptor, which act as autoreceptors by sensing the neurotransmitter content at the synaptic cleft and eliciting feed-back mechanisms (Engelman and MacDermott, 2004). In addition, postsynaptic depolarization can lead to the release of retrograde messengers (e.g. dopamine, glutamate, BDNF, oxytocin, and endocannabinoids) from the postsynaptic compartment to modify presynaptic properties (Kombian et al., 1997; Nagappan and Lu, 2005; Nugent et al., 2007; Zilberter, 2000). The regulation of neurotransmitter release probability seems to play an important role as high-pass- and low-pass filters during information processing (Abbott and Regehr, 2004).

Long-term plasticity refers to mechanisms which result in synaptic remodeling that persist for hours, days, and longer (Citri and Malenka, 2008). Hence, these mechanisms are crucial for the development of neuronal circuits and for information storage as long-term memory (Segal, 2005). The two most studied phenomena of long-term plasticity are long-term potentiation (LTP) and long-term depression (LTD). Both have been primarily investigated at glutamatergic synapses in the CA1 region of the hippocampus, which involve activation of $\mathrm{N}$ methyl-D-aspartate (NMDA) receptors (NMDARs) (Lüscher and Malenka, 2012; Martin et al., 2000). Important for the induction of LTP and LTD are the different channel properties of $\alpha$ amino-3-hydroxy-5-methyl-4-isoxazole propionic acid (AMPA) receptors (AMPARs) and NMDARs, the two major ionotropic glutamate receptors at excitatory synapses (Benke et al., 1998; Mayer et al., 1984). Activation of AMPARs by presynaptic glutamate release leads to an influx of the monovalent cations sodium and potassium and generates the excitatory postsynaptic response. NMDARs, in contrast, are blocked by magnesium at resting membrane potential and this block is only released upon strong depolarization of the postsynaptic membrane and simultaneous activation by glutamate (1984; Nowak et al., 1984). If this occurs, sodium and calcium can pass the NMDAR channel into the postsynaptic compartment. It is believed that the level of calcium within the dendritic spine regulates the switch between depression and potentiation (Malenka and Nicoll, 1993). Highfrequency synaptic stimulation leads to a strong increase in calcium concentration and the induction of LTP, whereas repetitive low-frequency stimulation causes a moderate increase in calcium and finally LTD (Sabatini et al., 2002). A crucial switch might be the timing of presynaptic action potential input and the backpropagating potential coming from the dendrites. LTP is evoked if the presynaptic spike slightly precedes the backpropagating 
action potential at the synapse, and vice versa for LTD (Stuart et al., 1997; Waters et al., 2005). Calcium inside the dendritic spine triggers a number of signaling events leading to rapid changes in postsynaptic AMPAR content ('early-phase' response) and with some delay to local dendritic protein synthesis and induction of gene expression in the nucleus ('latephase' response) (Greer and Greenberg, 2008; Henley and Wilkinson, 2016). The latter will be discussed in more detail below.

\subsubsection{Synapse-to-nucleus signaling}

Long-term potentiation is thought to be a molecular correlate of learning and memory and long-term memory consolidation requires 'late-phase' LTP including gene expression (Adams and Dudek, 2005). This excitation-transcription coupling makes it inevitable that the signal generated during synaptic transmission is propagated to the nucleus in order to induce the expression of activity-dependent genes. The communication between the synapse and the nucleus is of great importance and multiple routes have been identified (Bading, 2013; Nakamura et al., 1999; Xia et al., 1996) (illustrated in figure 1). As mentioned earlier, neurotransmitter release at glutamatergic synapses can elicit a calcium influx into the postsynaptic compartment through the opening of the NMDAR channel. Although this rise in calcium concentration through NMDARs mainly remains restricted to the dendritic spine, several lines of evidence indicate that calcium is the key second messenger for communication between synapse and nucleus (Bading et al., 1997; Chawla, 2002; Greer and Greenberg, 2008; Redmond, 2008; Sheng and Greenberg, 1990). Furthermore, experiments with nuclear calcium indicators demonstrated that changes in nuclear calcium concentration correlate with synaptic activity and specific quenching of nuclear calcium prevented the induction of $43 \%$ of all activity-dependent genes (Bengtson et al., 2010; Hardingham et al., 1997; Zhang et al., 2009). Thus, by which mechanisms does the calcium signal propagate to the nucleus and what are alternative routes? The first mechanism involves voltage-gated calcium channels (VGCC). Upon membrane depolarization those channels open and calcium fluxes into the cell (Fatt and Katz, 1953; Llinás et al., 1976). Calcium influx through VGCCs located in the membrane of the cell soma and the dendrites rapidly increases the intracellular calcium concentration caused by a steep gradient across the membrane (McBurney and Neering, 1987). Calcium can enter the nucleus by diffusion and activate downstream effectors such as calcium/calmodulin-dependent protein kinase IV (CaMK4). Through phosphorylation, CaMK4 passes the signal to cAMP-responsive elementbinding protein (CREB), one of the key activity-dependent transcription factors, and its interaction partner CREB-binding protein (CBP) (Chawla et al., 1998; Impey et al., 2002). Together, they induce expression of a huge set of activity-dependent genes (e.g. FOS, ARC, $B D N F$ ) (Kim et al., 2010; Pfenning et al., 2007). Recent data suggest that the CaMK2 
subunits alpha, beta, and gamma are playing an important role for inducing neuronal plasticity-coupled gene expression as well. In this model, calcium influx through the VGCC Cav1.2 first mobilizes actin-bound CaMK2 $\alpha / \beta$ and, subsequently, a voltage-dependent conformational change of the channel causes an accumulation of CaMK2 $\alpha / \beta$ (Li et al., 2016). This in turn activates CaMK2 $\gamma$ by phosphorylation and CaMK2 $\gamma$ shuttles calcium-loaded calmodulin (CaM) into the nucleus where it induces CREB-CBP activation via CaMK4 (Ma et al., 2014). Voltage-dependent calcium channels are not the only regulators of intracellular calcium concentrations. The calcium signal might be amplified from the endoplasmic reticulum (ER), which can function as a high capacity calcium store (Berridge, 1998). For instance, ryanodine receptors can release calcium from the ER (Kuwajima et al., 1992). In addition, inositol triphosphate (IP3) receptors line up at the ER membrane along the dendrites and sequential receptor activation might generate a propagating calcium wave through calcium release from the ER. Activation of IP3 receptors occurs when synaptic transmission activates G-protein coupled receptors (GPCRs) which stimulate the generation of IP3 and diacylglycerol (DAG) via phospholipase C (PLC) (Jaffe and Brown, 1994; Nakamura et al., 1999; Watanabe et al., 2006). After a calcium wave has occurred, cytoplasmic calcium concentrations are brought back to a low level by exporting calcium through calcium ATPases and sodium-calcium exchangers located at the plasma membrane and via sarcoendoplasmic reticulum calcium ATPases into the ER (Verkhratsky, 2004).

CREB-dependent gene expression upon synaptic stimulation may also be induced by cAMP, another prominent second messenger (Mayr and Montminy, 2001; Montminy, 1997). It is produced by adenylate cyclases and the activation of some adenylate cyclases is dependent on calcium/CaM signaling (e.g. Adyc8) (Nicol and Gaspar, 2014). cAMP activates proteinkinase A (PKA) thereby stimulating CREB-mediated transcription. Pharmacological and genetic perturbations of the CAMP pathway in vivo caused deficits in cognitive tasks, thus establishing a link between CAMP signaling and learning and memory (Wang and Storm, 2003). However, to what extend cAMP signaling is contributing to activity-dependent gene expression remains unclear.

Calcium flux through NMDARs and VGCCs also activates the mitogen-activated protein kinase (MAPK) cascade including the key players ERK1/2 (Dolmetsch et al., 2001; Hardingham et al., 2001; Xia et al., 1996). Besides calcium/calmodulin-dependent kinases and PKA, MAPKs are the third kinase class that is important for long-term synaptic plasticity (Shalin et al., 2006; Sindreu et al., 2007). Entry-point for the MAPK pathway is the small GTPase Ras and evidence suggests that Ras is activated by Ras guanyl-nucleotide releasing factors (Ras-GRFs) complexed with calcium-loaded CaM (Ebinu et al., 1998; Farnsworth et al., 1995). Ras activity in the postsynapse is controlled by the CaMK2dependent Ras-GTPase activating protein SynGAP, a risk gene for intellectual disability, epilepsy and autism spectrum disorders (ASD) in humans (Jeyabalan and Clement, 2016). 
Active Ras induces indirectly the phosphorylation of Raf isoforms (e.g. ARAF) which in turn phosphorylate MEK1/2. Unphosphorylated MEK1/2 traps ERK1/2 in the cytoplasm, but phosphorylation releases ERK1/2 and activates it. ERK1 and ERK2 have multiple cellular substrates, such as ribosomal protein S6 kinase 2 (RSK2), mitogen- and stress-activated kinase 1 (MSK1), and Elk-1 a transcriptional co-factor of serum-response-factor (SRF) (Arthur et al., 2004; Gille et al., 1995; Xing et al., 1996). Activation of MAPK-signaling upon synaptic stimulation is reported to have local and global effects on neuronal function (Wiegert and Bading, 2011). As pointed out earlier, calcium influx through synaptic NMDARs remains locally confined to the dendritic spine. This is also observed for activated ERK $1 / 2$ at the synapse which have been shown to participate in the regulation of AMPAR cycling between the PSD and endosomes (Kim et al., 2005; Zhu et al., 2002). In addition, MAPK signaling at the soma and inside the nucleus is also required for synaptic plasticity and memory formation (Shalin et al., 2006; Sindreu et al., 2007). However, little is known how synaptic activity is connected to active ERK1/2 in the soma and nucleus. A possible scenario may involve a rise in somatic and nuclear calcium concentrations upon opening of VGCCs leading to a global activation of MAPK signaling (Adams and Dudek, 2005; Wiegert et al., 2007). A cytoplasmic substrate of activated ERK1/2 is for example RSK2 which induces CREB-dependent gene expression. Besides its role in transcription factor activation, nuclear ERK1/2, together with MSK1, also regulates activity-dependent gene expression more broadly by chromatin remodeling through histone 3 phosphorylation (Brami-Cherrier et al., 2007).

At last, the induction of activity-dependent genes occurs downstream of the presented signaling pathways from the synapse to the nucleus. Description of the activity-dependent transcriptome and epigenome by RNA-seq and ChIP-seq, respectively, provided insight into the transcriptional program underlying long-term synaptic plasticity (Kim et al., 2010; Malik et al., 2014; Zhang et al., 2009). The transcriptional response itself has multiple layers. Signals from the synapse are activating pre-existing transcription factors (e.g. CREB, MEF2, SRF) and chromatin remodeling factors (West et al., 2002). This regulates the expression of immediate-early-genes (IEGs), a class that contains multiple transcription factors itself (e.g. FOS, EGR1, c-JUN) (Murphy et al., 1991). Subsequently, those IEG transcription factors induce the expression of late-response genes (e.g. BDNF, HOMER1) which function at the synapse and thus exert long-lasting modulations of synaptic contacts in neuronal circuits during LTP and LTD (Barco et al., 2005; Sala et al., 2003). Recent findings indicate that particularly the late response gene expression differs between excitatory- and inhibitory neurons due to different sets of active enhancer regions (Spiegel et al., 2014). How exactly differential signaling and the combinatorial action of various transcription factors dictate the cellular response to experience-driven activity remains elusive and requires further investigation. Furthermore, it is likely that so far unrelated protein networks directly or 
indirectly contribute to the regulation of neuronal function and thus represent an untouched pool of potential targets for drug discovery in the field of central-nervous system (CNS) diseases.

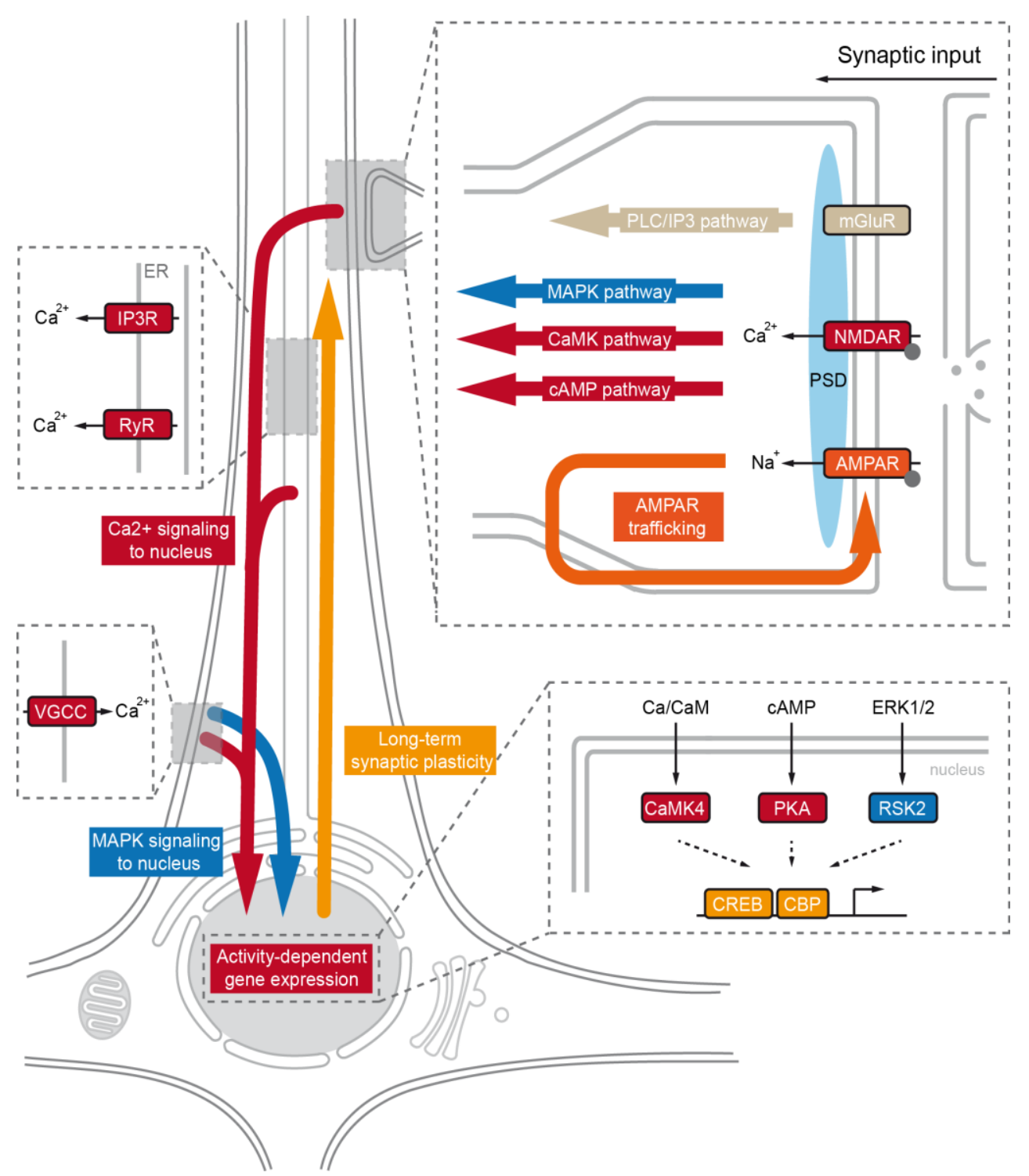

Figure 1: Regulatory pathways in synaptic plasticity and activity-dependent gene expression.

Presynaptic release of glutamate can initiate multiple signaling cascades in the postsynaptic neuron. Trafficking of AMPARs at the postsynapse directly modulates synaptic strength during LTP and LTD. Long-term synaptic plasticity requires activity-dependent gene expression of synaptic proteins. Calcium is thought to be the main second messenger from the synapse to the nucleus, for instance via calcium/calmodulin-dependent kinases (CaMK). The calcium signal might be enhanced through calcium influx from the ER or by voltage-gated calcium channels (VGCC) located in the plasma membrane. Kinases, such as CaMK4, PKA, and RSK2, phosphorylate various regulators of the activity-dependent gene expression program, like CREB and CBP (for citations see main text). Adapted from (Bading, 2013; Ebert and Greenberg, 2013). 


\subsection{Synaptic dysfunction as a converging point of psychiatric diseases}

The etiology of psychiatric disorders like schizophrenia, bipolar disorder (BPD) and ASD remains in large parts unknown although great progress has been made over the last decade in the field of brain imaging and risk factor identification (Ripke et al., 2013; Sarkar et al., 2015). In addition, neurons established from induced pluripotent stem cells (iPSC) represent a new source to explore molecular and cellular processes that might be altered in patients (Haggarty et al., 2016). A substantial body of evidence from various methodologies suggests that synaptic dysfunction and calcium-signaling play a central role in multiple psychiatric diseases (West and Greenberg, 2011). The following sections review findings at different levels starting with alterations in neuronal circuit function and ending with the multitude of genetic mutations accounting for disease susceptibility.

\subsubsection{Evidence at the circuit level}

Studies that aimed to decode the structural and functional connectivity of the brain using imaging (functional MRI) and electrophysiological (EEG/MEG) techniques suggest that the brain connectome has small-world topology (Uhlhaas and Singer, 2012). This implicates that neuronal micro-networks in spatially distant brain areas communicate via few highly connected hubs (Bullmore and Sporns, 2009). Communication between prefrontal cortex and hippocampus, for instance, is thought to be required for information processing and long-term storage (Brincat and Miller, 2015; Igarashi, 2015) Such higher brain functions are dependent on various kinds of oscillatory activity of neuronal groups and their synchronization over short and long distances (Varela et al., 2001). While long-range communication rather involves theta $(4-7 \mathrm{~Hz})$, alpha $(8-12 \mathrm{~Hz})$, and beta $(13-25 \mathrm{~Hz})$ oscillations, local oscillatory activities tend to be at higher gamma-band frequencies (25-200 $\mathrm{Hz}$ ) (von Stein and Sarnthein, 2000). Electrophysiological studies with schizophrenia patients have revealed reduced cortical gamma-oscillations during cognitive tasks like working memory (Haenschel et al., 2009) (Figure 2). In addition, altered theta- and alpha-oscillations have been recorded during sensory-gating in patients with schizophrenia (Hong et al., 2010). Oscillatory activity is the result of a precisely tuned interplay between groups of excitatory neurons and inhibitory interneurons within neuronal circuits (Wang and Buzsáki, 1996). In the cerebral cortex such circuits are formed by excitatory glutamatergic pyramidal cells and inhibitory GABAergic interneurons (Whittington et al., 1995). Those inhibitory neurons, in particular fast-spiking parvalbumin (PV) positive interneurons, are responsible for the finetuning of gamma-frequency oscillations during cognitive tasks (Bartos et al., 2007). The physiological basis for impaired high-frequency gamma-oscillations in schizophrenia patients is therefore likely a dysbalance between excitation and inhibition (E/I) (Sohal et al., 2009). 
This causality is supported by various studies in pharmacological rodent models for symptoms of schizophrenia. Administration of the NMDA receptor antagonists Ketamine or MK-801 into the prefrontal cortex of mice and rats leads to decreased evoked gammaoscillations and finally a schizophrenia-like phenotype (Homayoun and Moghaddam, 2007a; Saunders et al., 2012). Notably, it has been observed that a NMDA receptor hypofunction leads to reduced inhibitory control of glutamatergic output neurons, resulting in cortical excitation (Homayoun and Moghaddam, 2007b). Finally, optogenetic elevation of the E/I balance within the prelimbic/infralimbic cortex of mice caused impairments during learning and social behavior paradigms (Yizhar et al., 2011). Taken together, these findings indicate that the modulation of glutamatergic neurotransmission might be relevant for the treatment of schizophrenia and ASD.

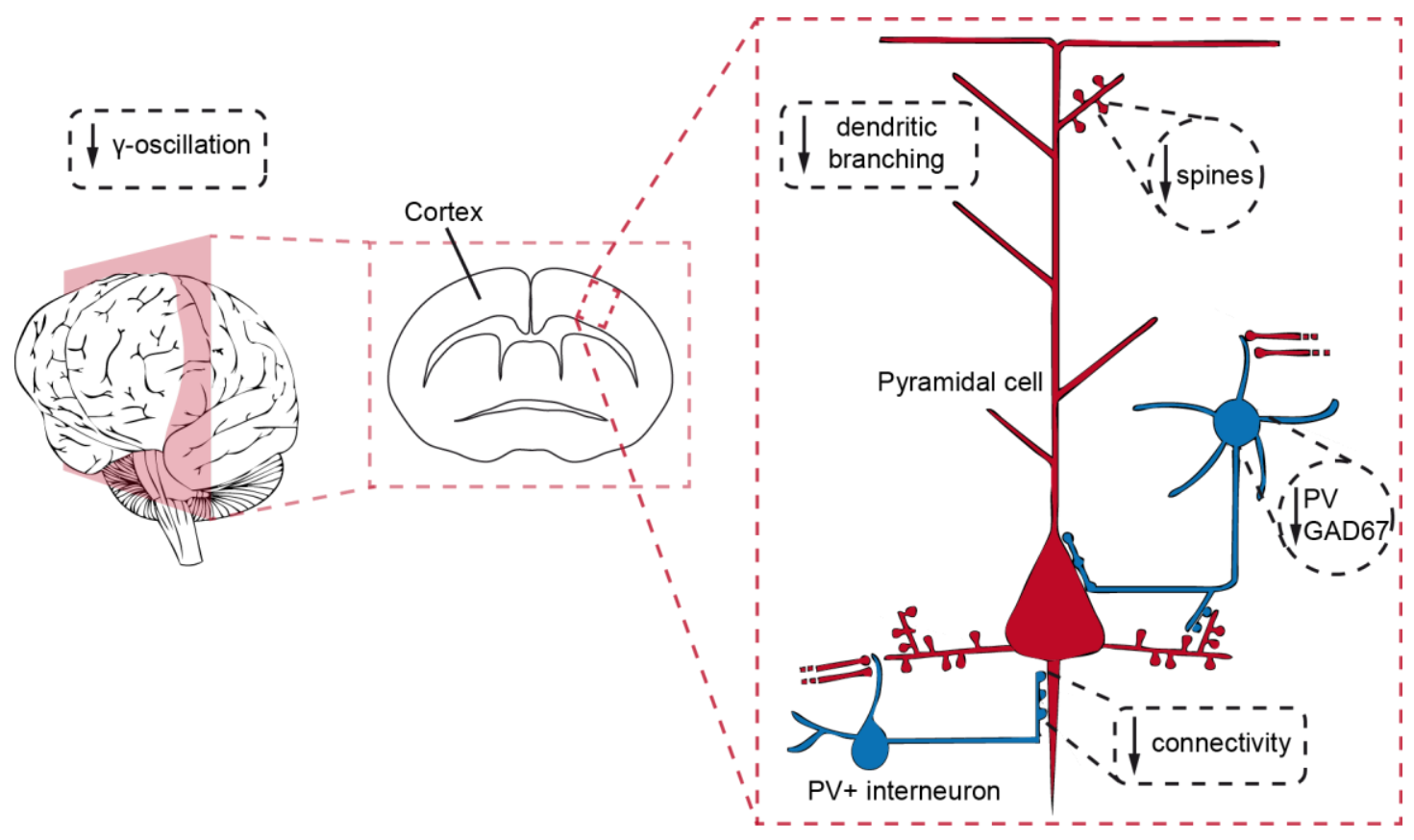

Figure 2: Alteration in cortical circuit function in schizophrenia.

Schizophrenia patients are showing morphological and cellular hallmarks of disturbed neurodevelopment and circuit formation such as decreased dendritic arborization, reduced spine density, and decreased interneuron-marker (PV, GAD67) expression (for citations see main text). Adapted from (Marín, 2012; Pratt et al., 2012).

\subsubsection{Evidence at the cellular level}

At the cellular level multiple studies provide evidence for alterations in neuronal maturation and synaptic plasticity in schizophrenia patients (Penzes et al., 2011). Schizophrenia subjects show shorter and less branched dendrites (Black et al., 2004; Guidotti et al., 2000; 
Selemon and Goldman-Rakic, 1999). Furthermore, a reduced spine density has been observed on pyramidal neurons of the primary auditory cortex and on CA3 dendrites of the hippocampus (Kolomeets et al., 2005; Steen et al., 2006; Sweet et al., 2009) (Figure 2). Until recently, psychiatric research was strongly limited by the unavailability of neuronal material from patients and restricted to postmortem tissue and pharmacologic as well as genetic mouse models. The seminal work by Takahashi and Yamanaka, who for the first time reprogramed human fibroblasts into iPSCs, paved the way towards patient-derived induced neurons (iNeurons) (Takahashi and Yamanaka, 2006). Such iNeurons harbor the complex genetic burden of the patient and thus represent a unique cellular model to study neuropathology. A study by Brennand and colleagues provided the first evidence that iNeurons established from schizophrenia patients reflect cellular correlates of the disease (Brennand et al., 2011). Those cultures showed reduced connectivity compared to iNeurons from healthy controls and decreased expression of synaptic proteins like PSD-95 and glutamate receptor subunits (GRIK1, GRIK4, GRM7, GRIN2A). In addition, dysregulated cAMP and WNT signaling activity was detected (Brennand et al., 2011). Moreover, altered energy metabolism has been reported for schizophrenia patient-derived neuronal progenitor cells and glutamatergic neurons derived from patients suffering from BPD (Mertens et al., 2015; Paulsen et al., 2013). Cultures from patients with a DISC1 (Disrupted-in-Schizophrenia 1) loss-of-function mutation showed neurodevelopmental abnormalities, synaptic deficits and gene expression changes (Wen et al., 2014).

Taken together, current evidence from various sources of morphological studies highlight that schizophrenia, BPD, and ASD are most likely neurodevelopmental disorders with a significant synaptopathology. As a consequence, disturbed neuronal circuit function and unbalanced $E / I$ are detected, which seem to be putative causes for symptoms like cognitive deficits, social isolation, and hallucination. A major challenge still is to decipher the link between genetic susceptibility for psychiatric disorders and the occurrence of symptoms as well as the response to medications. Psychiatric genomics made great progress in the identification of risk loci over the last years and current knowledge will be discussed in the following chapter.

\subsubsection{Evidence at the molecular level}

In schizophrenia and BPD both genetic and environmental factors have an impact on disease vulnerability. Family and twin studies have estimated a heritability of $\sim 80 \%$ and $\sim 60 \%$ for schizophrenia and BPD, respectively (Song et al., 2015; Sullivan et al., 2003) (Figure 3). Attempts to link genetic variations to schizophrenia by classical human genetics delivered only few solid associations. Worth mentioning in that context are the 22q11.2 
micro-deletion and the involvement of DISC1. The 22q11.2 micro-deletion, which in most of the cases occurs de novo, results in the disruption of 30-60 genes on chromosome 22 and leading to a high risk of $25 \%$ to develop schizophrenia (Bassett et al., 2008; Karayiorgou et al., 2010). DISC1 was linked to schizophrenia in a genetic study of a Scottish family that had an unusual number of cases with mental illness (St Clair et al., 1990). A chromosomal translocation was detected which disrupts the DISC1 gene. Great progress has been made in the field of psychiatric genetics since the advent of genome-wide-association-studies (GWAS) and in particular with the collaborative approach by the Psychiatric Genomics Consortium (PGC). This permitted screening of a sufficient number of patients and healthy controls to detect associations between common variants (frequency $>1-5 \%$ in the population) and psychiatric disorders at genome-wide significance (Cross-Disorder Group of the Psychiatric Genomics Consortium et al., 2013; Ripke et al., 2013). It became apparent that the genetic background of schizophrenia is highly heterogeneous and polygenic (Schizophrenia Working Group of the Psychiatric Genomics Consortium, 2014). Thus, so far undetermined combinations of multiple genetic variants seem to build the genetic vulnerability, where each variant only has a small effect size (Franke et al., 2016). In the case of schizophrenia the latest release from the PGC describes 108 loci which reach genome-wide significance by comparing 36,989 cases with 113,075 controls (Schizophrenia Working Group of the Psychiatric Genomics Consortium, 2014) (Figure 3). Since most of those loci lie within regulatory and not exonic regions, usually the proximal genes are reasoned to be involved in disease susceptibility (Maurano et al., 2012). The GWAS data is an excellent basis for studies on disease etiology and drug discovery for psychiatric disorders and it is now required to analyze putative risk genes in greater depth. This might finally bridge the gap between genetic susceptibility and the clinical symptoms. As a first step, genes at or near associated loci for schizophrenia have been analyzed whether they converge into common biological processes. The most recent releases of the PGC report on an enrichment of genes affecting calcium signaling (e.g. CACNA1C, CACNAB2, CACNA11), glutamatergic transmission and synaptic plasticity (e.g. GRM3, GRIN2A, SRR, GRIA1) and targets of MIR137 (e.g. TCF4) (Ripke et al., 2013; Schizophrenia Working Group of the Psychiatric Genomics Consortium, 2014). Furthermore, the major histocompatibility complex (MHC) locus, comprising over 200 genes with known functions in T-cell immune response, has been the first and since then the strongest genetic risk association for schizophrenia (Charles A Janeway et al., 2001; Stefansson et al., 2009). Overall, enhancers that are active in tissues with immune function harbored an enriched number of schizophrenia associations, however, it has long been elusive what kind of role these common variants play in the pathophysiology of schizophrenia (Ripke et al., 2013; Schizophrenia Working Group of the Psychiatric Genomics Consortium, 2014). Recently, complement component 4 (C4), a member of the classical component cascade, was identified as the main schizophrenia risk 
gene at the MHC locus and a function in synapse elimination during postnatal development has been reported (Sekar et al., 2016).

Similar to the blurred boundaries between symptomatic manifestations of various psychiatric disorders, genetic data is challenging the distinction as the genetic correlations between schizophrenia, BPD and major depressive disorder (MDD) is in the range of 0.4-0.6 (Maier et al., 2015). Besides common variants, rare copy number variations (CNVs), which are less frequent but have higher penetrance, seem to play an important role in disease etiology for schizophrenia and also for ASD (Figure 3). Such structural variants (SV), including microdeletions and microduplications of more than 500 kilobases, affect the expression of dozens of genes (Levinson et al., 2011; Vacic et al., 2011). Figure 3 summarizes the current data for heritability, GWAS loci, and structural variants for four major mental illnesses (Geschwind and Flint, 2015).
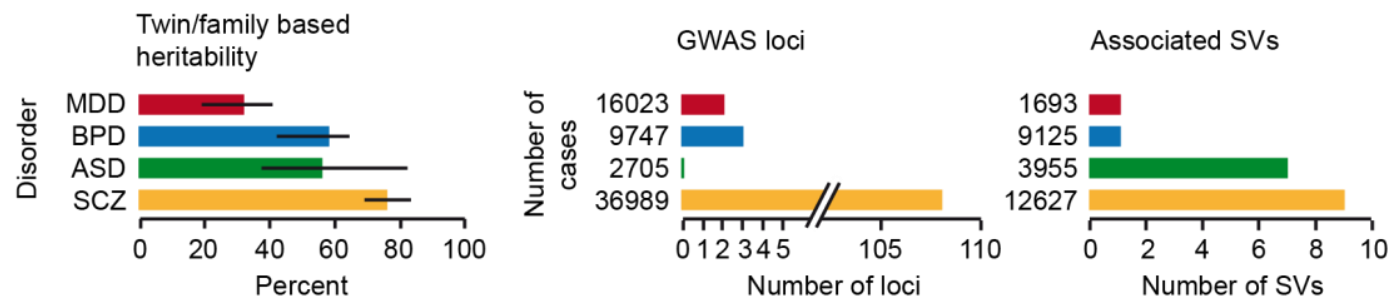

Figure 3: Summary of genetic associations for MDD, BPD, ASD, and SZD.

Left, estimated heritability from twin and family studies. Errorbars (sem). MDD, major depressive disorder; BPD, bipolar disorder; ASD, autism spectrum disorder; SCZ, schizophrenia. Middle/right, the number of identified genome-wide significant loci and associated structural variants (SV). The number of analyzed patients within the largest study for a disorder is given on the $y$-axis. Adapted from (Geschwind and Flint, 2015).

A systematic detection of rare variants (allele frequency $<0.5-1 \%$ ) within protein-coding regions by exome sequencing (exome-seq) is now possible, as costs for next-generationsequencing are falling. However, similar to GWAS, large sample numbers are inevitable to gain statistical power (Tennessen et al., 2012). Two recent large scale exome-seq projects have identified a significant enrichment of disruptive rare variants within sets of voltagegated calcium ion channel genes (e.g. CACNA1C, CACNA1B, CACNA1H), genes of the post-synaptic density (PSD), and genes of the ARC-complex and the NMDAR-complex (e.g. DLG1, DLG2, CaMK2A/2B, SLC25A3, ABLIM1, SYNGAP1) (Fromer et al., 2014; Purcell et al., 2014). In addition, target genes of the ASD-associated RNA-binding protein FMRP, which controls translation at the synapse, have been enriched significantly (Darnell et al., 2011; Tang et al., 2015). The concept that many psychiatric disorders primarily represent synaptopathies is further supported by a thorough characterization of the human synapse 
proteome. A mapping to known disease-associated mutations revealed that over 200 genes connected to the synapse cause changes in synapse physiology, and are related to over 130 human brain diseases (Bayés et al., 2011).

In conclusion, genetic studies on psychiatric disorders are revealing the highly complex polygenicity for disorders like schizophrenia and BPD. The genetic associations are enriched within synaptic genes, genes involved in calcium signaling and immune function and the overlap, both in terms of genetics as well as symptoms, is high for schizophrenia, BPD, and MDD. Hence, it is likely that assay development for research on neuronal function will have an impact on a broad range of CNS diseases in general, and neuropsychiatric disorders in particular.

\subsection{Inventory of the high-throughput toolbox in neuroscience}

Development of high-throughput methods for applications in neuroscience is generally lagging behind other biomedical research areas like oncology. This is not surprising as the cellular systems as well as the phenotypes of interest are highly specialized. Nonetheless, immense progress has been made to increase, for example, the throughput through advanced high-content assays, but pooled genetic assays are still absent from the highthroughput toolbox in neuroscience.

\subsubsection{Cellular systems}

Neurons are highly specialized cells which is reflected by the complexity of regulatory physiological processes and how frequent perturbations lead to pathological states. Given its unique repertoire of cellular functions it is inevitable that the regulation of neuronal excitation and synapse-to-nucleus signaling needs to be studied in a differentiated and mature neuron population. This has also been only recently acknowledged by the pharmaceutical industry after suffering a series of failures during clinical trials for drugs that originated from classical biochemical target-based drug discovery projects (Scannell and Bosley, 2016). In this scenario, the predictive validity of an in vitro assay, which is the ability to yield good answers and good treatments based on, for example, profiles of cellular activities, is strongly dependent on the biological relevance of the cell culture system (Vincent et al., 2015).

Primary neurons from mice and rats are for a long time a well-established cellular system to study neuronal differentiation and signaling in the dish. Neuronal maturation in this system is relatively fast, resulting in an extensive network with functional synaptic connections already at day-in-vitro (DIV) 12-15 (Figure 4) (Baj et al., 2014). At that time, spontaneous network 
activity can be recorded and synaptic activity can be strongly induced by blocking the inhibitory input of the network using the $\mathrm{GABA}_{A}$-receptor antagonist bicuculline (BIC). In addition, for functional genomics studies, it is of advantage that sufficient cell numbers of high purity can be obtained from embryonic animals.

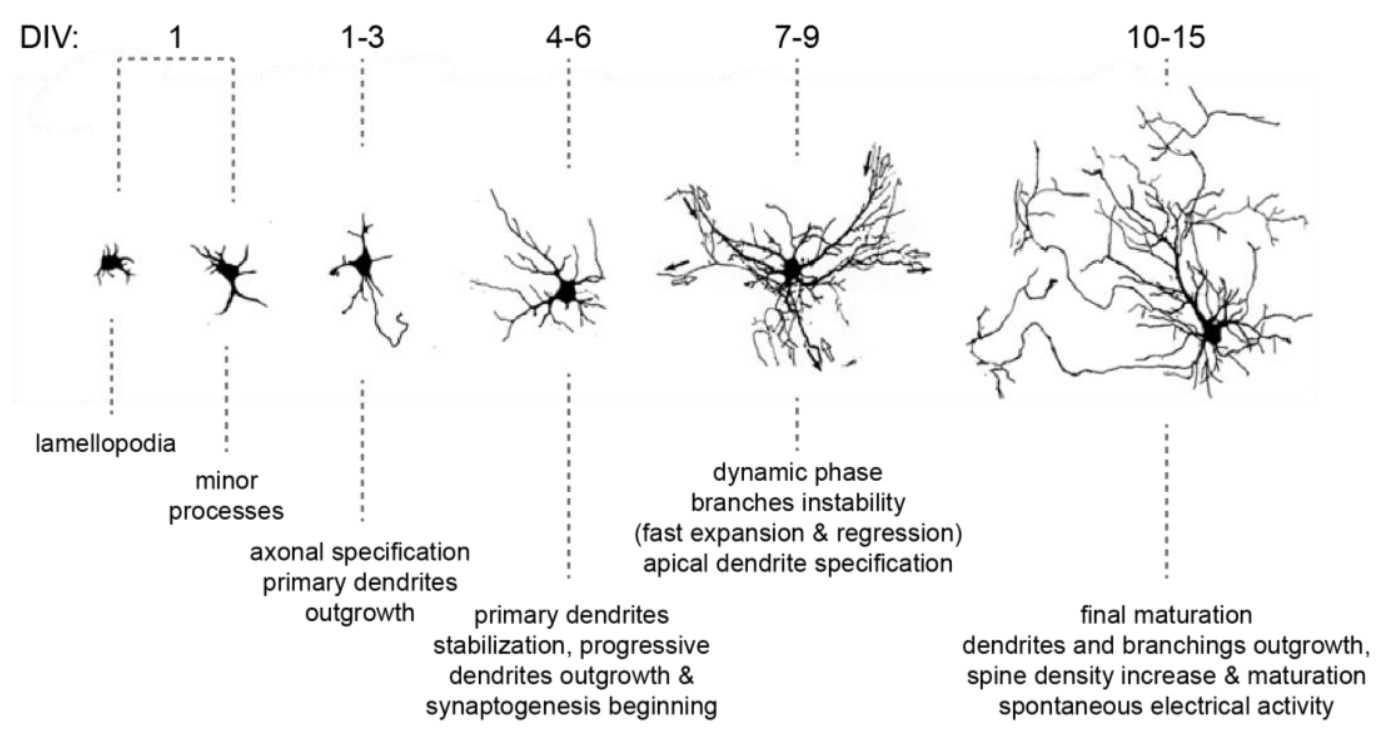

Figure 4: Maturation stages of murine primary neurons.

Maturation stages of primary neuron from DIV 1 till 15 . Staging was done using murine primary hippocampal neurons, but is most likely similar for cortical neuron cultures. Within two weeks mature neuronal cultures are generated. Adapted from (Baj et al., 2014).

An alternative and highly valued source for neuronal cell culture assays are iNeurons, which have been differentiated from iPSCs of a given human donor (Dimos et al., 2008). This technique has attracted much attention as it allows studying cell biology of a neuron that harbors the genetic information of the donor (Mertens et al., 2016). Thus, it represents a unique source for cells with the highly complex set of genetic variations present in a psychiatric patient (Brennand et al., 2011; Habela et al., 2016; Haggarty et al., 2016; Krey et al., 2013; Topol et al., 2015; Wen et al., 2014). However, this method is relatively new and a variety of iNeuron-differentiation protocols are available which strongly differ in cell yield, purity, and maturation state of the culture (Mertens et al., 2016). In general, human-derived iNeurons tend to differentiate and mature much slower compared to rodent primary neurons and a decent extent of functionally mature synapses is usually only achieved by co-culture with astrocytes over several weeks (Nicholas et al., 2013). Nevertheless, differentiation protocols are improving rapidly and as soon as yield, purity, and the number of functional 
synapses have increased, iNeurons will likely become the cell-type of choice for functional genomics screens in neuropsychiatric research.

\subsubsection{High-content screens}

High-content assays are one of the most advanced high-throughput assays in general and in particular in neuroscience. Their power lies in the multi-parametric analysis of cellular phenotypes using automated microscopy (Boutros et al., 2015). However, assay design is not trivial as the molecular correlates of a given phenotype need to be stained with high specificity or transgenic labels must be available in order to enable robust segmentation of cellular features by automated image analysis. The most frequently used marker is neuronal viability and thus neuroprotection has been the main focus so far (Anderl et al., 2009; Schulte et al., 2011). More sophisticated parameters are neurite length and arborization as well as changes in synapse number (Harrill et al., 2011; Ofengeim et al., 2012; Radio, 2012). In the last years significant progress has been made in order to streamline and facilitate the image acquisition (Caicedo et al., 2016). This has led to a growing number of users and the pharmaceutical industry is using high-content assays in phenotypic drug discovery for CNS diseases (Haggarty et al., 2016; Hunsberger et al., 2015). While high-content compound screens are feasible with neuronal cultures, genetic interference screens are more challenging (Harrill et al., 2011; Ofengeim et al., 2012). A reason is the poor transfectability of primary neurons, which as a consequence implies the use of viral transduction. However, the generation of large-scale arrayed viral libraries for the expression of thousands of shRNAs (short hairpin RNA) or cDNAs is extremely expensive and laborious and usually not feasible for a single academic group. To my knowledge, only one single high-content RNAi has been published until now with primary neurons (Nieland et al., 2014). This screen used a relatively small scale arrayed lentiviral RNAi library of 607 shRNA vectors to screen for regulators of synaptogenesis. High-content assays and pooled genetic screens can be considered somewhat as complementary. A high-content screen using the top hits from a pooled genetic screen may, for example, represent a very powerful orthogonal secondary validation tool.

\subsection{Functional genomics}

Functional genomics is a research field that is investigating the impact of the genotype on molecular or cellular phenotypes at genome-scale. This involves for example the study of dynamic changes in the transcriptome, the proteome, and the epigenome as well as largescale loss- and gain-of-function studies. In recent years functional genomics approaches in 
neuroscience have delivered a wealth of data about the neuronal activity-dependent transcriptome and epigenome, as well as the cellular and synaptic proteome (Bayés et al., 2011; Kim et al., 2010; Malik et al., 2014; Sharma et al., 2015; Zhang et al., 2009). Systematic large-scale approaches to study gene function during neuronal activity are, however, missing.

\subsubsection{Genetic perturbation by RNA interference and CRISPR-Cas9}

Understanding gene function is one major quest in molecular biology. Early after the discovery of the post-transcriptional mRNA abundance control mechanism called RNAi in the nematode Caenorhabditis elegans in 1998 and later in mammals, RNAi became the prime method for loss-of-function studies (Jinek and Doudna, 2009; Winter et al., 2009)(Fire et al., 1998). The endogenous RNAi pathway takes place within the nucleus and the cytoplasm and involves two major cleavage steps (illustrated in figure 5). miRNAs are typically transcribed as gene-clusters by RNA polymerase II (Borchert et al., 2006; Lee et al., 2004). The generated transcripts, termed primary miRNA (pri-miRNA), are capped, polyadenylated, and usually comprise several hundred nucleotides in length. Pri-mRNAs fold into characteristic hairpin structures containing imperfectly base-paired stems (Carthew and Sontheimer, 2009). The fold of the transcript is important for its proper processing. The first of the two major maturation steps occurs within the nucleus where the stem-loop (hairpin) of the pri-miRNA is cleaved-off by a microprocessor complex containing the RNase III enzyme Drosha (Lee et al., 2003). This cleavage occurs co-transcriptionally and generates the miRNA precursor (pre-miRNA) which is $~ 70 \mathrm{nt}$ in length and consists of a stem which harbors the crucial $\sim 22 \mathrm{nt}$ miRNA sequence and a terminal loop. The pre-miRNA is exported into the cytoplasm by the transport factor Exportin-5 in complex with Ran-GTP (Yi et al., 2003; Zeng and Cullen, 2004). Thus, the second major processing step occurs in the cytoplasm and involves the multi-protein RISC loading complex (RLC) (Gregory et al., 2005). The RLC consists of the RNase III Dicer, the double-stranded RNA-binding proteins TRBP (Tar RNA binding protein). PACT (protein activator of PKR), and the core component Argonaute-2 (Ago2). The terminal loop of the pre-miRNA is cleaved-off by Dicer, creating the mature 22 nt long miRNA duplex (Chendrimada et al., 2005). This double-stranded RNA consists of the guide strand and the passenger strand. While the guide strand contains the sequence complementarity to the mRNA target and thus has to be used for gene regulation, the passenger strand must be degraded. After cleavage of the pre-miRNA, Dicer and its interactors TRBP and PACT dissociate from the miRNA duplex. The active RNA-induced silencing complex (RISC) is formed by an asymmetric guide strand selection by Ago2 (Frank et al., 2010; Khvorova et al., 2003; Schwarz et al., 2003). The active RISC is then guided to its mRNA target mainly by position 2 to 8 of the guide strand which is defined as the seed 
region of the miRNA (Rajewsky, 2006). The extent of complementarity of miRNA and mRNA are thought to be a key determinant of the regulatory mechanism. While a perfect match induces Ago2-mediated cleavage of the mRNA, central mismatches rather promote repression of mRNA translation (Filipowicz et al., 2008).

The usage of RNAi for loss-of-function studies is easy and fast. Usually, short doublestranded RNA molecules are transfected into cells where they are processed into small interfering RNAs (siRNAs) of about 22 nucleotides in length. These siRNAs can silence gene expression in a sequence specific manner. A second strategy is based on the expression of shRNAs, an endogenous-like early intermediate RNA of the RNAi pathway. These shRNAs can be expressed from RNA polymerase II promoters or RNA polymerase III promoters (e.g. human U6 promoter) (Li et al., 2007; Mohr et al., 2014). This feature was a critical milestone for the development of pooled RNAi screens, as it allows viral delivery and stable shRNA expression over long cultivation periods. The principle of pooled genetic screens will be discussed in chapter 2.4.3. 


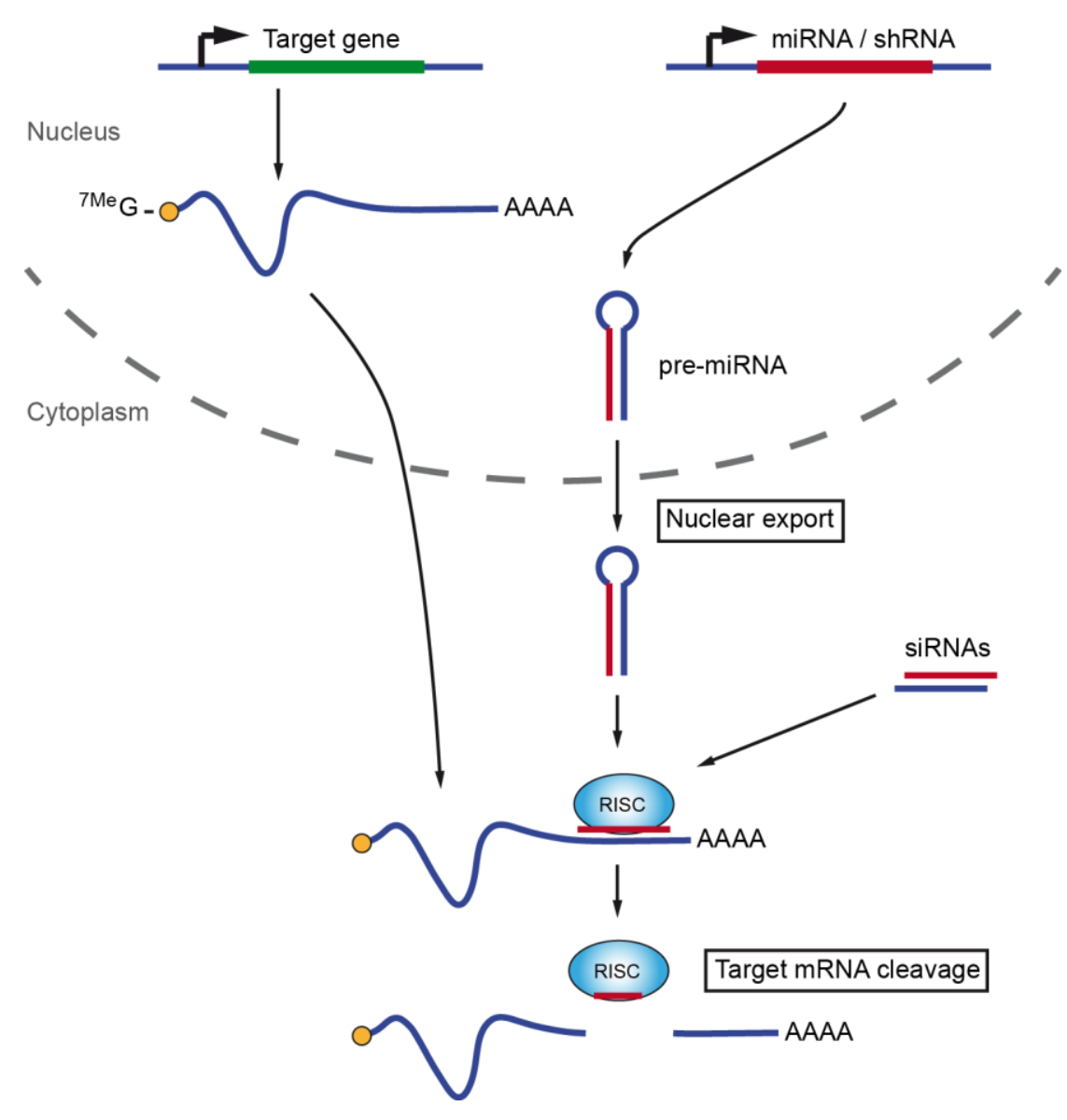

\section{Figure 5: Gene silencing by RNAi}

Exogenous RNAi reagents like shRNAs and siRNAs can enter the endogenous RNAi pathway at different steps. shRNAs are expressed from plasmids or viral genomes and mimic precursor miRNAs (pre-miRNAs). Therefore, they need to be processed by the RNase III Dicer (not shown) before the guide RNA (in red) gets incorporated into the RNA-induced silencing complex (RISC). siRNAs are RNA duplexes which can be delivered by transfection. They directly enter RISC, but only allow transient gene silencing. In case of perfect complementarity of the guide strand to the target $\mathrm{mRNA}$, the target transcript is cleaved. Otherwise, translational repression is triggered by a partial complementarity (not shown). Adapted from (Mohr et al., 2014).

In 2011, a groundbreaking publication by Charpentier and colleagues described how the prokaryote Streptococcus pyogenes uses clustered regularly interspaced short palindromic repeats (CRISPR) as a defense mechanism against invading prophage DNA (Deltcheva et al., 2011). This mechanism has evolved into a new tool for genome editing and gene expression regulation (Jinek et al., 2013). The CRISPR locus of Streptococcus pyogenes is transcribed into a precursor CRISPR RNA (pre-crRNA), which is processed by RNase III enzymes into short CRISPR RNAs (crRNA). A second small RNA, called trans-activating crRNA (tracrRNA), brings the crRNA and the Cas endonuclease together to form a trimeric complex. Guided by the crRNA towards invading prophage-DNA, Cas protein cleaves the foreign DNA and thereby protects the bacterium (Deltcheva et al., 2011). Somewhat 
afterwards, additional work figured out that Cas9 is the only Cas protein required for this mechanism and that the tracrRNA and the crRNA can be fused into a single short guide RNA (sgRNA) (Jinek et al., 2012). The sgRNA harbors a 20 nucleotide long sequence complementary to the target DNA and a hairpin-forming region for Cas9 binding. Cas9 itself contains two critical endonuclease domains ( $\mathrm{HNH}$ and RuvC-like), which cleave both strands of the target DNA (Jinek et al., 2012). Another important feature of the system is the protospacer-adjacent-motif (PAM). The PAM represents just a few bases adjacent to the target sequence and is recognized by Cas 9 directly. Studies on other prokaryotes revealed that CRISPR-Cas is a conserved bacterial defense system and each Cas9 orthologue has its own PAM sequence specificity (Leenay et al., 2016). Thus, on-target specificity results from the interplay between sgRNA-target complementarity and the PAM sequence. The indepth knowledge about sgRNA and Cas9 function made the CRISPR-Cas9 system the nextgeneration tool for genome engineering. To date a variety of applications using CRISPRCas9 exist (Hsu et al., 2014). The most classical is to induce a double-strand break at the gene-of-interest in order to trigger non-homologous end-joining which can lead to a gene knock-out or to create a knock-in by homologous recombination of a donor DNA fragment (Figure 6A) (Jinek et al., 2013). Characterization of the endonuclease domains of Cas9 led to Cas9 mutants (D10A, H840A) without endonuclease activity, so called dead-Cas9 (dCas9) (Qi et al., 2013). The dCas9 variant can be used to shuttle additional effector domains to a specific genomic locus (Figure 6B). By fusing transcriptional activators (e.g. VP64) or repressors (e.g. KRAB) to Cas9 and targeting the fusions close to the transcriptional-start-site it is possible to regulate the expression of virtually any gene-ofinterest. These applications are also referred to as CRISPR activation (CRISPRa) and CRISPR interference (CRISPRi) (Gilbert et al., 2014; Larson et al., 2013). Recent advancements allow enhancing the regulatory effect through targeting multiple effector domains to a single locus (Konermann et al., 2015; Tanenbaum et al., 2014). One interesting tool is the synergistic-activation-mediator (SAM) system, that uses engineered sgRNAs (sgRNA2.0) containing two additional hairpin-structures (MS2 stem-loops), which act as RNA aptamers and bind the corresponding RNA-binding domain (MCP domain) (Konermann et al., 2015). Multiple effector domains can be fused to the RNA-binding domain for strong regulatory effects (Figure 6C). 
A

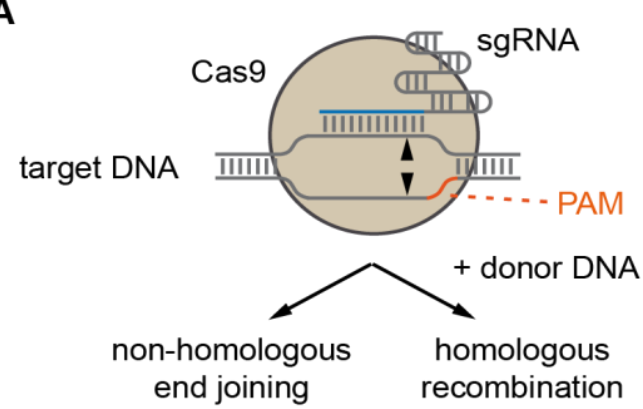

B

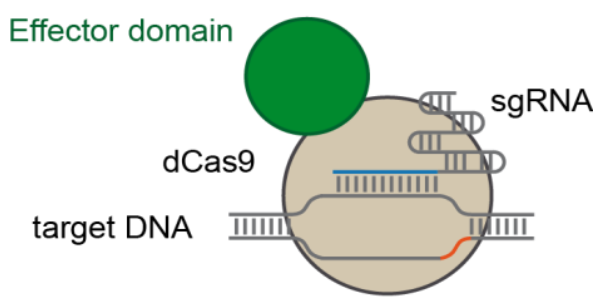

C

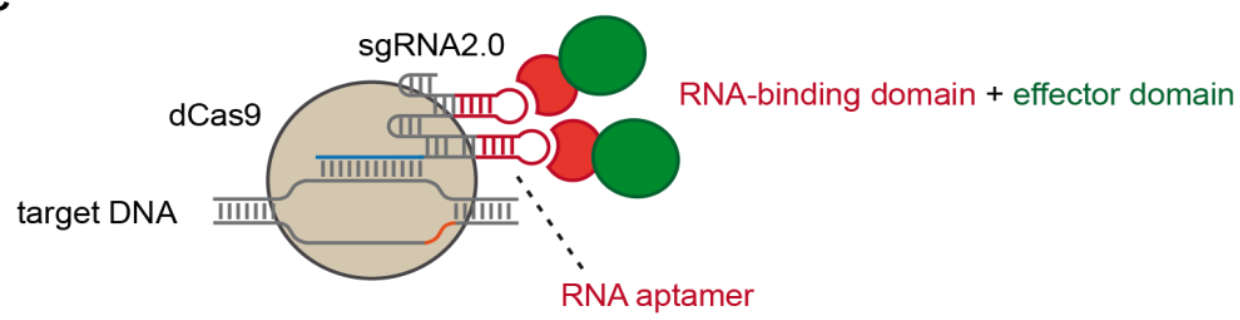

Figure 6: Overview of CRISPR-Cas9 applications

A. Ternary complex of Cas9 protein, sgRNA and target DNA including the PAM sequence. Cas9 cleaves the target DNA at both strands (arrowheads). The cell will repair this double-strand break either by non-homologous end-joining or if a donor DNA fragment is present by homologous recombination. B. Fusion of an effector domain to Cas9 without endonuclease activity (dCas9) allows visualization (e.g. GFP fusion) or gene expression regulation (e.g. VP64 or KRAB fusion) at a specific genomic site. C. The SAM system recruits effector domains via sgRNAs containing RNA aptamers (e.g. MS2 stem-loop) that recruit proteins containing specific RNA-binding domains (e.g. MCP). Modified from (Shalem et al., 2015).

\subsubsection{Advances through molecular barcoding and its application}

The ability of viral delivery and long-term expression of shRNAs was not the only important milestone towards pooled genetic screenings. A second major advancement was the development of oligonucleotide arrays and later the progress in next-generation sequencing (NGS). Oligonucleotide arrays are glass slides onto which thousands of defined singlestranded DNA oligonucleotides are printed using a high-definition inkjet DNA synthesis procedure. Prior to next-generation-sequencing, such arrays have been used as microarrays to study differential gene expression by hybridization of fluorescently labeled cDNA to the arrayed immobilized oligonucleotides (Schena et al., 1995). Notably, it is also possible to release the synthesized oligonucleotides from the glass slide, thereby creating an enormous pool of synthetic DNA bricks for gene synthesis or large-scale shRNA/sgRNA and molecular barcode libraries (Bassik et al., 2009; Collins et al., 2009; Tian et al., 2009). Currently, all academic and commercial pooled shRNA/sgRNA libraries have been created by parallelized on-chip synthesis of custom designed shRNA or sgRNA sequences. 
Molecular barcoding describes a methodology which uses unique synthetic DNA sequence tags, coupled to a second functional genetic element, for an unambiguous parallelized readout. Already over 20 years ago this method was used to analyze bacterial virulence genes in a parallelized manner by insertional mutagenesis of transposons carrying a DNA barcode (Hensel et al., 1995). Only slightly later, a similar approach has proven to be useful for the generation of a library of yeast gene-deletion mutants covering $96 \%$ of all annotated Saccharomyces cerevisiae open reading frames. Each deleted gene was replaced by a DNA barcode sequence to identify individually deleted strains within a pooled quantitative survival assay (Giaever et al., 1999, 2002; Winzeler et al., 1999). Years later, the concept of a pooled loss-of-function assay was brought into mammalian cells by using pooled lentiviral shRNA libraries (Ngo et al., 2006; Paddison et al., 2004; Schlabach et al., 2008; Silva et al., 2008). Here, either a DNA barcode linked to the shRNA or one half of the shRNA hairpin was read in order to deconvolute the pooled assay. In the early days this was done using DNA microarrays and later by next-generation sequencing.

A second application for molecular barcodes are multiplexed cis-regulatory reporter assays. In this approach, RNA barcode reporters, which are under control of regulatory promoter elements (e.g. enhancers, transcription-factor binding sites, minimal promoters), replace classical protein reporters like GFP and luciferase. This enables tremendous multiplexing and principally activity measurements of all annotated cis-regulatory elements in a single experiment. Barcoded reporters have been first used to profile pathway activities downstream of ERBB signaling, an approach named EXTassay (Botvinnik et al., 2010). Later by the use of on-chip oligonucleotide synthesis of thousands of enhancer fragments, it was used as massively-parallel reporter assays (MPRAs) to dissect the activities of enhancers (Melnikov et al., 2012). During this project, a pooled cis-regulatory reporter gene assay has been used to compare the response of multiple barcoded sensors to synaptic activity.

\subsubsection{Pooled RNAi/CRISPR-Cas9 screenings}

Pooled genetic screens were invented to circumvent the highly laborious, time-consuming and expensive working steps associated with arrayed screens. As pointed before, pooled screens allow rapid generation of viral shRNA libraries for loss-of-function studies at genome-wide scale. Additionally, well-to-well variability is eliminated since all knockdowns are examined under the very same experimental conditions.

To date, three different screening paradigms exist. These have been very much influenced by the needs in cancer research, as first screens were performed in this field and still the majority of published screens are related to cancer (Ngo et al., 2006; Paddison et al., 2004; 
Schlabach et al., 2008; Silva et al., 2008). The three screening paradigms are hereafter called 'rescue screen', 'drop-out screen', and 'FACS-based screen' and the key steps are illustrated in figure 7 (Shalem et al., 2015). A rescue screen aims to discover genes which confer resistance to a cytotoxic treatment. Hence, those genes are potentially required for sensitivity to the treatment. In drug discovery, this assay is frequently used to identify the target or mode-of-action (MoA) of a compound (Deans et al., 2016; Fennell et al., 2014; Wagner and Schreiber, 2016). The second paradigm, a drop-out screen, is performed to identify essential genes for cell survival and proliferation. As the name suggests, its goal is to remove those cells from the test population where the knockdown impairs viability (Shalem et al., 2015). This usually requires long-time culture periods in order to gain sensitivity. An interesting and often used subtype of a drop-out screen is a synthetic-lethal screen, a method aiming to unravel combinatorial effects of the knockdown and a second genetic or pharmacological perturbation, for example an oncogenic mutation or a compound (Luo et al., 2009). FACS-based genetic interference screens represent a class that differs from the first two paradigms by its ability to study phenotypes other than cell viability or proliferation. Here, the cell pool is treated with a stimulus and the cellular response might be captured by an endogenous marker or a fluorescent reporter. The cell pool is then sorted into bins of strongand weak-responding cells. If a knockdown is interfering with signal propagation of the stimulus to the downstream marker or reporter, the corresponding cells are expected to be enriched in the weak-responding cell population (Parnas et al., 2015). Conversely, cells where the knockdown results for example in an enhanced response to the stimulus should be rather enriched in the strong-responding cell pool. This paradigm has been used recently to dissect the response of dendritic cells to bacterial LPS (lipopolysaccharide) at unprecedented accuracy (Parnas et al., 2015). In all three paradigms, the genomic DNA of the cell pools is extracted and the shRNA/sgRNA abundances are determined by nextgeneration sequencing. In rescue- and drop-out screens, the endpoint sample is usually compared to an initial reference sample for the identification of hits. In FACS-based screens, the strong- and weak-responding cell pools are compared directly for hit nomination.

Overall, pooled genetic interference screens have demonstrated its value for the discovery of new putative drug targets, MoA identification and pathway deconvolution. Recently, the methodology made an important move from the dominating cancer field to primary cells (Parnas et al., 2015). However, to date, no pooled screening strategy has been described for post-mitotic primary neurons to identify regulators of neuronal plasticity. 
(1)

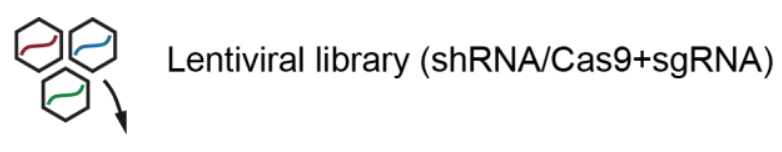

(2)

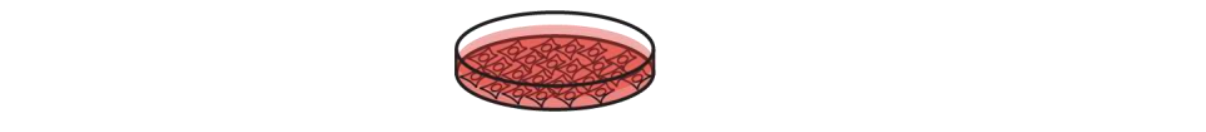

(3)

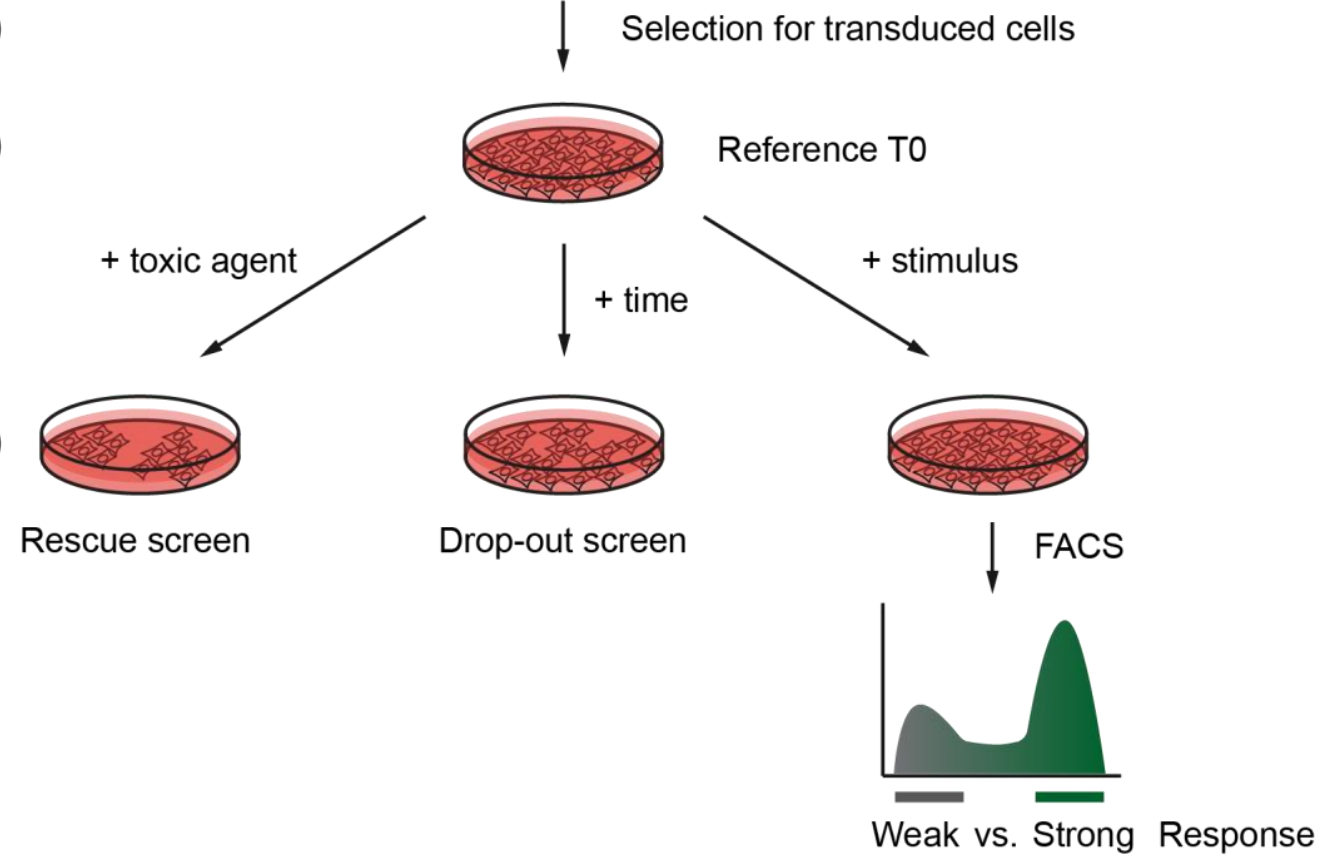

(4)

Rescue screen

Drop-out screen

Weak vs. Strong Response

FACS-based screen

\section{Figure 7: Current paradigms for pooled genetic screens.}

Three general paradigms for pooled genetic screens have been described: (i) rescue- , (ii) dropout-, and (iii) FACS-based screens. All methods share steps of infecting cell pools with a lentiviral shRNA/sgRNA library (step 1) and selecting for positively transduced cells (step 2). The cell pool after selection represents the reference sample (step 3). For a rescue screen, cells are treated with a toxic agent and only cells with a shRNA/sgRNA-mediated resistance remain in the cell pool (step 4, left). A drop-out screen can identify essential genes as the cells with the corresponding perturbation are lost over time from the population (step 4, middle). In a FACS-based screen, cells are stimulated and sorted based on their response (step4, right). Finally, relative shRNA/sgRNA abundances will be determined by NGS. For rescue- and drop-out screens, endpoint samples are compared to reference samples, and for FACS-based screens, the strongly responding candidates are compared to the weakly responding ones (for citations see main text). 


\subsection{Objectives}

Pooled RNAi screens represent a powerful tool to investigate gene function at the systems level. Its full potential to dissect any relationship between genotype and phenotype has so far only partially been addressed. The lack of innovative strategies to study phenotypes other than cell survival or proliferation precluded the development of resourceful experimental approaches for a long time. The aim of this thesis is to break this deadlock by combining the throughput of a pooled RNAi screen with the sensitivity of a genetic sensor that regulates the defined expression of molecular RNA barcode reporters. The hypothesis is that this approach could broaden the application spectrum of pooled genetic screens in terms of celltypes and the cellular phenotype of interest in general. For a proof-of-concept study primary neurons were selected as a challenging and meaningful cellular system that is of particular relevance for plasticity-related as well as neurodegenerative disorders. Moreover, primary cultured neurons are, because of technical reasons, a mainly untouched landscape for highthroughput assays in the field of drug discovery. Genome-wide association studies in psychiatric disorders, like schizophrenia, BPD, and ASD, have uncovered a plethora of risk genes that mainly converge into pathways involved in synaptic plasticity and calciummediated synapse-to-nucleus signaling. Current neuropharmacology is, however, only focusing on a few targets, for example the dopamine 2 receptor in the case of antipsychotics. Hence, it was the aim to develop an assay that can dissect the networks underlying neuronal excitation-transcription coupling, which in the future hopefully brings new potential drug targets into focus. In addition, psychiatric drug discovery is increasingly focusing on hit identification by phenotypic compound screens. Those assays might have a better predictive validity than classical biochemical assays, but the protein target of a drug is often unknown. Thus, there is a strong need for assays that allow target identification and pathway activity deconvolution. Pooled RNAi screen are already used for this purpose in different disease areas and the sensor-coupled pooled RNAi screen might enable target identification of drugs for CNS diseases.

At the time when the project was launched RNAi was the well characterized gold-standard technique for loss-of-function studies in cell culture systems. Recent studies of CRISPRCas9-mediated genetic perturbation argue to integrate this tool into the barcoded sensor assay introduced here. A process that has been initiated in this thesis. 


\section{Materials}

\subsection{Equipment}

$\begin{array}{ll}\text { Arium 611 Water Purification System } & \text { Sartorius } \\ \text { Picodrop Spectrophotometer } & \text { Picodrop Limited } \\ \text { Vortex Genie 2 } & \text { Bender + Hobein } \\ \text { Mini Centrifuge Model sprout } & \text { Biozym } \\ \text { Heraeus Centrifuge Fresco 17 } & \text { Thermo Fisher Scientific } \\ \text { Heraeus Megafuge 16 } & \text { Thermo Fisher Scientific } \\ \text { Thermomixer BioShake iQ } & \text { Biometra } \\ \text { Thermocycler T3000 } & \text { Biometra } \\ \text { Thermocycler TGradient } & \text { Biometra } \\ \text { Rotor-Gene Q cycler } & \text { Qiagen } \\ \text { Electrophoresis power supply } & \text { Pharmacia LKB } \\ \text { UV Gel documentation system } & \text { INTAS } \\ \text { Genepulser Xcell Elektroporationsgerät } & \text { BioRad } \\ \text { Ultra-low temperature lab freezer U725 } & \text { New Brunswick Scientific } \\ & \\ \text { Cell culture } & \\ \text { Hera Cell incubator } & \text { Thermo Fisher Scientific } \\ \text { Hera Safe Workbench } & \text { Thermo Fisher Scientific } \\ \text { Nalgene freezing Container "Mr. Frosty" } & \text { Sigma-Aldrich } \\ \text { Microscope Axiovert 25 } & \text { Zeiss } \\ \text { Microscope Observer Z1 } & \text { Zeiss }\end{array}$

\section{Luciferase measurements}

Tumbling Table WT17

Biometra

Microplate Reader Mithras LB 940

Berthold Technologies

32-Channel Luminometer LumiCycle 32

ActiMetrics

\section{Next-generation-sequencing}

Ion OneTouch2 System
Ion Personal Genome Machine (PGM)
Ion Proton
Ion Torrent Server
Ion Torrent Server
Qubit 2.0 Fluorometer
UV Airclean Workstation
Ion Torrent
Ion Torrent
Ion Torrent
Ion Torrent
Ion Torrent
Invitrogen
LTF Labortechnik 


\section{Software}

Microsoft Windows Professional 7

Microsoft Office 2007

Acrobat Reader 9.5

Illustrator CS5

Photoshop CS5

Lasergene 8.0

MicroWin 2000

Fiji ImageJ

R Version 3.2.3

R-studio Version 0.99.484

Tinn-R Editor Version 2.3.7.1

Lumicycle Version 1.4

Zotero

\subsection{Chemicals and consumables}

\subsubsection{Drugs used in experiments}

4-aminopyridine (4-AP)

D-APV

(-)-Bicuculline methiodide (BIC)

Glycine

Phorbol-12-myristat-13-acetat (PMA)

Rotenone

Strychnine hydrochloride

Tetrodotoxin (TTX)

\subsubsection{Antibodies and cell stains}

Rat anti-HA

Mouse anti-Flag-M2

Rabbit anti-synaptophysin

Mouse anti-Map2

Goat Alexa488 anti-mouse

Goat Alexa647 anti-rabbit

Goat Cy3 anti-rat

HRP-goat-a-mouse monoclonal lgG $(H+L)$
Microsoft

Microsoft

Adobe

Adobe

Adobe

DNA Star Inc.

Berthold Technologies

Freeware

Freeware

Freeware

Freeware

ActiMetrics

https://www.zotero.org
Abcam (ab120122)

Tocris (0106)

Abcam (ab120108)

Abcam (ab120050)

Sigma (R8875)

Abcam (ab120416)

Abcam (ab120055)
Roche (11867423)

Sigma (3165)

Abcam (ab52636)

Sigma (M1406)

Dianova (115-545-006)

Life Technologies (A21245)

Dianova 
HRP-goat-a-rat monoclonal $\lg G(\mathrm{H}+\mathrm{L})$

Prolong Gold Antifade reagent

Hoechst 33342

\subsubsection{Commercial kits}

Direct-zol RNA MiniPrep Kit

Qiagen AllPrep DNA/RNA Mini Kit

NucleoSpin Plasmid Quick Pure

NucleoBond PC100 Midiprep

NucleoSpin Gel and PCR Clean-up
Jackson Immuno Research Labs Jackson Immuno Research Labs Thermo Fisher Scientific Life Technologies

3.2.4. NGS Reagents \& Chips

Qubit dsDNA HS Assay Kit

Ion PGM Template OT2 200 Kit

Ion PGM Sequencing 200 Kit v2

Ion PGM Hi-Q ${ }^{\text {TM }}$ OT2 Kit

Ion PGM Hi-Q ${ }^{\text {TM }}$ Sequencing Kit

Ion $\mathrm{PI}^{\mathrm{TM}}$ Template OT2 200 Kit v3

Ion $\mathrm{PI}^{\text {TM }}$ Sequencing 200 Kit v3

Ion Sphere Quality Control Kit

Dynabeads MyOne Streptavidin C1

DNA LoBind Tubes (1.5 ml)

Ion 318 Chip Kit v2

Ion $\mathrm{PI}^{\mathrm{TM}}$ Chip Kit v2
Invitrogen

Ion Torrent

Ion Torrent

Ion Torrent

Ion Torrent

Ion Torrent

Ion Torrent

Ion Torrent

Invitrogen

Eppendorf

Ion Torrent

Ion Torrent

\subsubsection{Enzymes}

HotStarTaq Plus DNA Polymerase

Qiagen

PWO Polymerase

Roche

BP Clonase II

Thermo Fisher Scientific

LR Clonase II

Thermo Fisher Scientific 
LR Clonase II Plus

SuperScript III Reverse Transcriptase

TURBO DNase

Restriction enzymes

2x RotorGene SYBRgreen PCR Master Mix

NEBNext High-Fidelity 2x PCR Master Mix

T4 DNA ligase

Benzonase

Proteinase $\mathrm{K}$
Thermo Fisher Scientific

Thermo Fisher Scientific

Thermo Fisher Scientific

New England Biolabs

Qiagen

NEB

NEB

Sigma

Roth

\subsection{Eukaryotic cell lines}

PC12 tetOFF

Rat adrenal pheochomocytoma cell line expressing tetracycline regulated transactivator tTA (Clonetech)

HEK293wt

Human Embryonic Kidney immortalized cell line (ATTC)

HEK293FT

Human Embryonic Kidney immortalized cell line (ATTC)

SH-SY5Y

Human neuroblastoma cell line

N2a

Murine neuroblastoma cell line

\subsection{Bacterial strains}

Escherichia coli transformation competent cells:

MegaX DH10B electro-competent cells

Thermo Fisher Scientific

One Shot Mach1 chemical-competent cells Thermo Fisher Scientific

One Shot ccdB survival 2 T1R chemical-competent cells Thermo Fisher Scientific

Mach1 chemical-competent cells Self-made 


\subsection{Buffers and solutions}

AAV cell lysis buffer

$150 \mathrm{mM} \quad \mathrm{NaCl}$

$50 \mathrm{mM} \quad$ Tris- $\mathrm{HCl}(\mathrm{ph} 8.5)$

ICC blocking solution

$2 \mathrm{~g} \mathrm{BSA}$ in $80 \mathrm{ml} \mathrm{dH} 2 \mathrm{O}, 2 \mathrm{ml} \mathrm{FBS}, 2 \mathrm{ml}$ fishgelatine, $10 \mathrm{ml} \mathrm{10x}$ PBS

add up to $100 \mathrm{ml}$ with $\mathrm{dH} 2 \mathrm{O}$

\subsubsection{Solutions for western blotting}

Triton-X Lysis buffer

$50 \mathrm{mM}$ Tris $\mathrm{pH} 7.5$

$150 \mathrm{mM} \quad \mathrm{NaCl}$

$1 \% \quad$ Triton-X100

$1 \mathrm{mM} \quad$ EGTA

Protease inhibitors: Complete tablet (Roche), $10 \mathrm{mM} \mathrm{NaF}, 1 \mathrm{mM} \mathrm{ZnCl}$, $1 \mathrm{mM} \mathrm{Na}_{3} \mathrm{VO}_{4}, 4.5$ $\mathrm{mM} \mathrm{Na}_{4} \mathrm{P}_{2} \mathrm{O}_{7}$

\subsubsection{Solutions for luciferase assays}

Firefly luciferase assay buffer

$20 \mathrm{mM} \quad$ Tricine

$1.07 \mathrm{mM} \quad(\mathrm{MgCO} 3) 4^{*} \mathrm{Mg}(\mathrm{OH}) 2 * 5 \mathrm{H} 2 \mathrm{O}$

$2.67 \mathrm{mM} \quad \mathrm{MgSO} 4$

$0.1 \mathrm{mM}$ EDTA

33.3 $\mathrm{mM} \quad$ DTT 
$270 \mu \mathrm{M}$ Coenzyme A

$470 \mu \mathrm{M}$ D-Luciferin, free base

$530 \mu \mathrm{M}$ ATP

For dissolving of magnesium carbonate titrate the $\mathrm{pH}$ with $\mathrm{HCl}$ until the solution is clear. Then adjust the $\mathrm{pH}$ to 7.8 with $\mathrm{NaOH}$. Add luciferin and ATP at last and control the $\mathrm{pH}$. The buffer is stored at $-20^{\circ} \mathrm{C}$ in the dark and thawed at room temperature.

Renilla luciferase assay buffer

Prepare KxPO4 (pH 5.1) solution: adjust the $\mathrm{pH}$ of $1 \mathrm{M} \mathrm{KH} 2 \mathrm{PO} 4$ solution to 5.1 using $2 \mathrm{M}$ $\mathrm{KOH}$.

1.1 $\mathrm{M} \quad \mathrm{NaCl}$

$2.2 \mathrm{mM} \mathrm{Na2-EDTA}$

$0.22 \mathrm{M} \quad \mathrm{KxPO} 4(\mathrm{pH} 5.1)$

$0.44 \mathrm{mg} / \mathrm{ml} \quad$ BSA

$1.3 \mathrm{mM} \mathrm{NaN3}$

Adjust the $\mathrm{pH}$ to 5.0 with $\mathrm{KOH}$. Then add $1.43 \mathrm{mM}$ Coelenterazine (dissolved in $100 \% \mathrm{EtOH}$ ). The buffer is stored at $-20^{\circ} \mathrm{C}$ in the dark and thawed at room temperature.

\subsubsection{Solutions and media for cell culture}

NeuroCulture medium

Neurobasal medium, 2\% B27, 1\% GlutaMax

HEK293/N2a medium

$\operatorname{DMEM}(4.5 \mathrm{~g} / \mathrm{l}$ glucose), 10\% FBS, 1\% GlutaMax (maintenance medium)

$\operatorname{DMEM}(4.5 \mathrm{~g} / \mathrm{l}$ glucose), 1\% FBS, 1\% GlutaMax (starvation medium)

PC12 medium

DMEM(1 g/l glucose), 10\% FBS, 5\% HS, 1\% GlutaMax (maintenance medium) 
$\operatorname{DMEM}(1 \mathrm{~g} / \mathrm{l}$ glucose), $1 \%$ FBS, $1 \%$ GlutaMax (starvation medium)

SH-SY5Y medium

50\% DMEM(4.5 g/l glucose), 50\% F12, 10\% FBS, 1\% GlutaMax (maintenance medium)

50\% DMEM(4.5 g/l glucose), 50\% F12, 1\% FBS, 1\% GlutaMax (starvation medium)

\subsection{Oligonucleotide}

Oligonucleotides were made by the AGCTlab of the MPI of Experimental Medicine in Göttingen or purchased from Eurofins in Munich.

\section{cDNA synthesis:}

Oligo(dT)

Random nonamer (N9)

\section{qRT-PCR:}

Tcf4 fwd (Mm)

Tcf4 rev (Mm)

Arc fwd $(\mathrm{Mm})$

Arc rev (Mm)

Rpl13a fwd (Mm)

Rpl13a rev (Mm)

WPRE fwd

WPRE rev

hU6p fwd

hU6p rev

\section{lonTorrent sequencing:}

qDec1.2 fwd

qDec2.2. rev

Dec fwd

Dec rev

PGM_A_IXcodeX_AFA_S
PHO-тTтTтTтTTTтTTTTTTT

NNNNNNNNN

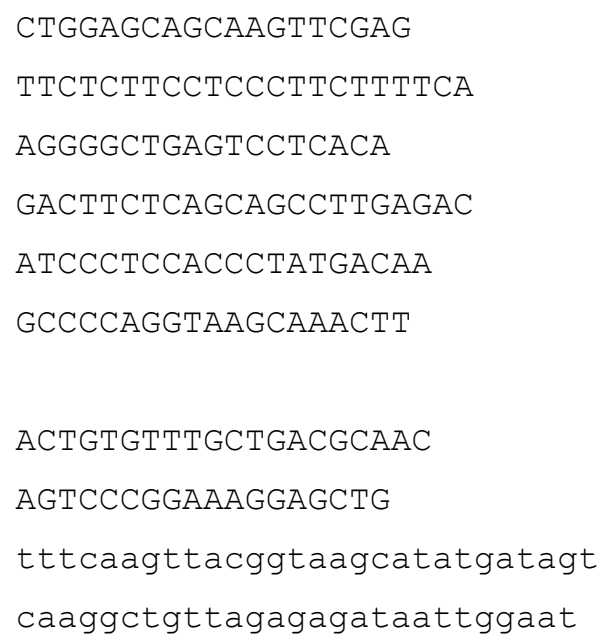


shRNA library cloning:

$\begin{array}{ll}\text { hU6 fwd } & \text { TCTCagagagagacagagacagatcc } \\ \text { Dec rev } & \text { GTAGACGCGAGTCAATGCATGTACGA } \\ \text { BC35 rev } & \text { GAGACttaagatATCGGATCCAGCTAGTTGC } \\ \text { SpA } & \text { GTAGACGCGAGTCAATGCATGTACGATCTAGACAATAAAAGATCTTT } \\ & \text { ATTTCATTAGATCTGTGTGTTGGTTTTTGTGTGACTAGTCTCGTA } \\ & \text { TGCCGTCTTTTGCTTGCC } \\ \text { BC35 } & \text { CttaagatATCGGATCCAGCTAGTTGCTAAGTCTGCCGAGTAGAATT } \\ & \text { AACCCTCACTAAAGGGTAGGTGACACTATHHHGHHHGHHHGHHHNN } \\ & \text { TVVVTVVVTVVVTVVVTCCTATAGTGAGTCGTATTAGTAGACGCGAG } \\ & \text { TCAATGCATGTACGATCTAGAC } \\ \text { PGM_trP1_hU6_as } & \text { CCTCTCTATGGGCAGTCGGTGATCttgtggaaaggacgaaacacC }\end{array}$

shRNAs:

\begin{tabular}{|c|c|}
\hline Tcf4 (target sequence) & TTCTAATTACCGGATATTGAAT \\
\hline \multirow[t]{2}{*}{ Adcy3 } & CCGGGCCATCTTTCTCAGGTTATTTGTTAATATTCATAGCAAATGACCT \\
\hline & GGGAAAGATGGCTTTTTT \\
\hline \multirow[t]{2}{*}{ II2rb } & CCGGCCCTCCAAACTTAATTATCCAGTTAATATTCATAGCTGGATGATT \\
\hline & AAGTTTGGAGGGTTTTTT \\
\hline \multirow[t]{2}{*}{ Camk2d } & CCGGGACGGGATGTTCTATGCAAATGTTAATATTCATAGCGTTTGCATG \\
\hline & GAACATCCCGTCTTTTTT \\
\hline \multirow{2}{*}{ Cacna1f } & CCGGCCCTCATCTACTGCTTAATAAGTTAATATTCATAGCTTATTGAGC \\
\hline & AGTAGATGAGGGTTTTTT \\
\hline \multirow[t]{2}{*}{ Bhlhe40 } & CCGGGTCAGCACAATTAAGTAAGAAGTTAATATTCATAGCTTCTTGCTT \\
\hline & AATTGTGCTGACTTTTTT \\
\hline \multirow[t]{2}{*}{ Calm1 } & CCGGGCCGCTATACTTCTTTATTATGTTAATATTCATAGCATAATAAAG \\
\hline & AAGTATAGCGGCTTTTTT \\
\hline \multirow[t]{2}{*}{ Tacr2 } & CCGGCCACAGGCAATGTTGATATAAGTTAATATTCATAGCTTGTATCAG \\
\hline & CATTGCCTGTGGTTTTTT \\
\hline \multirow[t]{2}{*}{ Gphn } & CCGGGCATACAAGATAGTACTAGATGTTAATATTCATAGCATCTGGTAC \\
\hline & TATCTTGTATGCTTTTTT \\
\hline \multirow[t]{2}{*}{ Disc1 } & CCGGGACTGGCTTATTTGAGAGAAAGTTAATATTCATAGCTTTCTCTCG \\
\hline & AATAAGCCAGTCTTTTTT \\
\hline \multirow[t]{2}{*}{ Cacna1h } & CCGGGCTAGAATGTAGTGAGGATAAGTTAATATTCATAGCTTATCCTCG \\
\hline & CTGCATTCTAGCTTTTTT \\
\hline
\end{tabular}


sgRNAs:

SV40-promoter

Arc-promoter

\section{Target sequence}

GAATAGCTCAGAGGCCGAGG

CCTACTCGCTCCCCTCCCGT

\subsection{Plasmids}

ID: AAV production:

V1739 pFdelta6 (adenoviral helper proteins)

V1740 pRV1 (serotype 2 capsid protein)

V1741 pH21 (serotype 1 capsid protein)

Luciferase assays:

V66 phRL-TK

cis-regulatory sensor assay:

V825 pAAVspace_DEST_luc2_WPRE

Cloning vector

1-6x SARE

pAAV_SARE-ArcMin-luc2_WPRE_pA

\section{PATHscreener:}

V1337 Cellecta Decipher Mouse Module 1 library

V1338 AAV E-SARE PATHscreener (with shRNA)

V1301 AAV E-SARE PATHscreener (shRNA stuffer)

\section{CRISPR-Cas9:}

V1785 pAAV_Syn1p-dCas9

V1787 PATHscreener2.0 (sgRNA stuffer)

sgRNA-SV40

pSV40-luc2
Library or single shRNA

Non-targeting control 


\section{Methods}

\subsection{Culturing of eukaryotic cell lines}

The cell lines PC12, SH-SY5Y, N2a, HEK293, and HEK293FT were cultured in the appropriate growth medium at $37^{\circ} \mathrm{C}$ in a humidified incubator at $5 \% \mathrm{CO}$. After reaching 80 $90 \%$ confluency, cells were passaged using standard protocol including treatment with $0.05 \%$ trypsin-EDTA. For all experiments, cells were seeded into the appropriate cell culture plate one day prior to the beginning of the experiment. Maintenance of the cell culture stocks, including passaging and test for mycoplasma contamination, has been done with the help of Beate Kauschat and Nadia Gabellini (Molecular Neurobiology, Department of Psychiatry, LMU Munich).

\subsection{Primary neuron culture}

Primary mouse cortical neurons were prepared from E15.5 C57BL/6 mice embryos. Culture dishes were freshly coated with poly-L-lysine ( $\mathrm{PLL}, 0.1 \mathrm{mg} / \mathrm{ml}$ in $\mathrm{dH} 2 \mathrm{O}$ ) overnight at room temperature. Dishes were washed twice with $\mathrm{dH} 2 \mathrm{O}$. For immunocytochemistry, acid-treated glass coverslips were placed into the culture dish and coating was done with PLL $(0.1 \mathrm{mg} / \mathrm{ml}$ in borate buffer). Coverslips were washed twice with PBS. Immediately after washing, half of the final culture medium volume of neuronal plating medium (containing $5 \%$ FBS) was added to the coated culture dishes and placed into a cell culture incubator at $5 \% \mathrm{CO} 2$. In case of 96-well plates, FBS was omitted from the initial plating in order to avoid the complete medium exchange on DIV1. Mouse cortices were dissected in cold HBSS/5 mM HEPES. Up to 16 pairs of cortices were dissociated in $2 \mathrm{ml}$ pre-warmed Neurobasal medium containing Papain $(20 \mathrm{U} / \mathrm{ml})$ at room temperature for $13 \mathrm{~min}$. Papain treatment was terminated by adding $10 \mathrm{ml}$ pre-warmed and $\mathrm{pH}$ equilibrated $\operatorname{DMEM}(4.5 \mathrm{~g} / \mathrm{l}$ glucose $) / 10 \%$ FBS. The medium was replaced once with new $10 \mathrm{ml}$ pre-warmed and $\mathrm{pH}$ equilibrated DMEM(4.5 g/l glucose) $/ 10 \%$ FBS and then with $2 \mathrm{ml}$ pre-warmed and $\mathrm{pH}$ equilibrated neuronal plating medium. Cells were gently triturated with a P1000 pipette. The cell suspension was transferred through a $40 \mu \mathrm{m}$ mesh (BD Cell strainer) to remove any cell clumps. Next, cell concentration was determined by counting trypan blue negative cells using a Neubauer cell counting chamber. Finally, cells were plated by adding the second half of the final culture medium volume to the cell culture dish. In all experiments a cell density of $\sim 500 \mathrm{cells} / \mathrm{mm}^{2}$ was used.

Except for experiments in 96-well plates, where serum-free medium was used from the beginning, culture medium was completely replaced by serum-free neuronal culture medium on DIV1. Feeding of the neuronal cultures was done on DIV6-7 for the first time by replacing 
half volume with pre-warmed and $\mathrm{pH}$ equilibrated neuronal culture medium. From then on, cultures were fed every 3-4 days until the end of the experiment.

\subsection{AAV production}

The production of AAV particles was done following the general published guidelines with the assistance of Beate Kauschat (Molecular Neurobiology, Department of Psychiatry, LMU Munich) (McClure et al., 2011). In detail, this included the following steps:

\section{Transfection}

AAVs were produced using HEK293FT cells. For a single virus preparation, $12 \mathrm{e}+06$ cells were seeded onto one PLL $(0.02 \mathrm{mg} / \mathrm{ml})$-coated $15 \mathrm{~cm}$ cell culture dish the day before transfection. One hour prior to the transfection, the cell culture medium was replaced by 15 $\mathrm{ml}$ HEK293 medium. The transfection mix was prepared as followed: Per $15 \mathrm{~cm}$ dish, $10 \mu \mathrm{g}$ pFdelta6, $3.75 \mu \mathrm{g} \mathrm{pRV1,} 3.75 \mu \mathrm{g} \mathrm{pH} 21$, and $4 \mu \mathrm{g}$ pAAV (i.e. the custom AAV plasmid) were mixed in $0.5 \mathrm{ml}$ OptiMEM medium. Polyethyleneimine (PEI) was used as a transfection reagent and added to the DNA/OptiMEM mix in a PEI:DNA ratio of $4: 1$. The transfection mix was thoroughly mixed and incubated at room temperature for 10 minutes. Next, the transfection mix was added drop wise to the cells. Four hours post-transfection, $15 \mathrm{ml}$ HEK293 medium were added. The mix of two AAV capsid expressing plasmids pH21 (serotype 1) and pRV1 (serotype 2) results in AAV particles with mixed capsid proteins form AAV serotype 1 and 2 . This mix was determined to have superior infection efficiencies compared to either serotype alone (data not shown).

\section{AAV harvest}

Three days post-transfection, AAV particles were harvested from the culture. Therefore, the cells were detached from the cell culture dish by rigorous pipetting and the cell suspension was transferred into a $50 \mathrm{ml}$ tube. Next, cells were pelleted by centrifugation at $1000 \mathrm{rpm}$ for 5 minutes and the supernatant was aspirated. The cell pellet was resuspended in $5 \mathrm{ml}$ of AAV cell lysis buffer and cells were lysed by three freeze-thaw cycles between $-80^{\circ} \mathrm{C}$ and $37^{\circ} \mathrm{C}$. In order to digest the genomic DNA, the cell lysate was incubated with benzonase (50 $\mathrm{U} / \mathrm{ml}$ ) at $37^{\circ} \mathrm{C}$ for 30 minutes. Subsequently, the cell lysate was centrifuged at $1000 \mathrm{rpm}$ for 15 minutes to pellet the cell debris. Afterwards, the AAV-containing supernatant was passed through a $0.45 \mu \mathrm{M}$ filter and transferred into an Amicon Ultra-15 centrifugal filter unit (100 $\mathrm{kDa}$ membrane cutoff, Millipore) in order to ultra-filtrate and concentrate the viral particles. During this procedure $10 \mathrm{ml}$ cold PBS was added twice to the AAV solution to exchange the buffer. The final volume after concentration was $0.25-0.5 \mathrm{ml}$ and aliquots of the virus were frozen and stored at $-80^{\circ} \mathrm{C}$ until usage. 
Absolute quantification of the AAV genomic copies (GC) by quantitative PCR (qPCR)

The quantification of the AAV GCs served as an approximate value to control the infection rate between experiments. For the quantification by $P C R$, the AAV genomes were first purified from the AAV preparation. Therefore, $5 \mu \mathrm{l} A \mathrm{AV}$ stock was mixed with $84 \mu \mathrm{ldH} 2 \mathrm{O}, 10$ $\mu l$ 10x Turbo DNase buffer and $1 \mu \mathrm{l}$ Turbo DNase $(2 \mathrm{U} / \mathrm{L})$ to digest residual pAAV plasmid DNA. The mix was incubated at $37^{\circ} \mathrm{C}$ for 15 minutes and the TurboDNase was subsequently inactivated at $95^{\circ} \mathrm{C}$ for 5 minutes. Next, $5 \mu \mathrm{l}$ proteinase $\mathrm{K}(10 \mathrm{mg} / \mathrm{ml})$ were added and incubated at $55^{\circ} \mathrm{C}$ for 10 minutes to digest the AAV capsid and release the AAV genomic DNA. The AAV genome was purified using the NucleoSpin Gel and PCR Clean-up kit (Macherey\&Nagel) and eluted in $200 \mu$ l elution buffer.

Absolute quantification was done on a Qiagen Rotor-Gene cycler using the 2x RotorGene SYBRgreen PCR Master Mix and the hU6p qRT-PCR primer pair. A pAAV plasmid serial dilution (1e+03 - 1e+06 copies/ $\mu$ l, plus non-template control) was used as a standard and always ran in parallel to the AAV genome sample. Final AAV GC titers in the range of $1 \mathrm{e}+09$ $1 \mathrm{e}+10 \mathrm{GC} / \mu \mathrm{l}$ were usually obtained.

\section{Determination of the infectious AAV titer}

In order to correlate the AAV GC titer to the infection rate of murine primary neurons, cortical cultures were infected with a serial dilution of a GFP expressing AAV and GFP positive cells were counted by a microscopic analysis (Figure 8). This correlation was used to adjust the infection rate of the AAV shRNA library to a level where it can be assumed that the great majority of infected cells are only transduced by a single AAV particle.

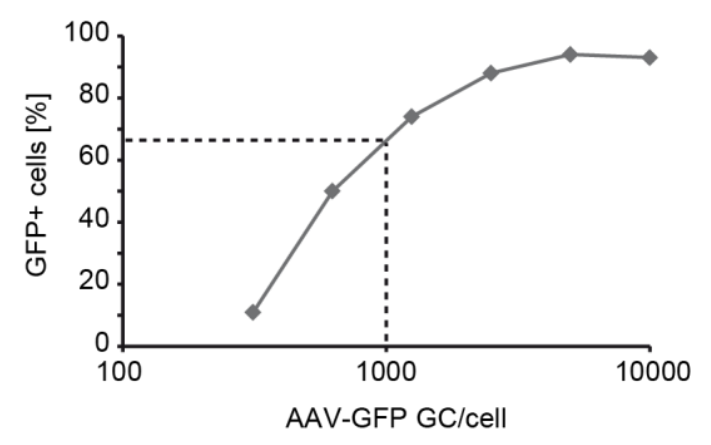

Figure 8: AAV infection rate

$A A V$ infection rate for primary neurons determined using a serial dilution of a GFP expressing AAV vector. 


\subsection{Rotenone dose-response cell viability assay}

The response of primary neurons to various rotenone concentrations was determined by microscopy counting of cell nuclei, as well as quantification of genomic DNA (gDNA) and total RNA. Primary neurons were seeded onto PLL-coated 6-well plates. Rotenone was diluted in half-logarithmic steps in DMSO as $100 x$ solutions (from $3.16 \mathrm{e}-07 \mathrm{M}$ to $3.16 \mathrm{e}+10 \mathrm{M}$ ) and $1 / 100$ volume of the final cell culture volume was added to the cells in duplicates or triplicates at DIV7. Cells were incubated at $37^{\circ} \mathrm{C}$ in a humidified incubator at $5 \% \mathrm{CO} 2$ until DIV14.

For the microscopic counting, cell nuclei were stained on DIV14 using Hoechst dye. Using a Zeiss Observer Z1 microscope, three images of the nuclear stain per well were acquired. Subsequently, the neuron cultures were lysed and gDNA as well as total RNA was isolated using the Qiagen AllPrep DNA/RNA Mini Kit. The gDNA and total RNA concentrations were determined using a UV spectrophotometer. The images of the nuclear stain were further analyzed using Fiji ImageJ software. For counting of all cell nuclei the Analyze Particle function was used, generating an output file with the individual nucleus areas. Discrimination between live and dead cells was done by applying a filter for pyknotic (dead) and nonpyknotic (live) nuclei. First, the mean nucleus areas of pyknotic and non-pyknotic nuclei were determined manually and then used as thresholds to filter the microscopic nuclei count. Dose-response curves of all measurements were done using the $\mathrm{R}$ package drc.

\subsection{Multiplexed cis-regulatory sensor assay}

Cloning of the cis-regulatory sensor library

The AAV-based cis-regulatory sensor library was cloned and provided by Dr. Ben Brankatschk (Molecular Neurobiology, Department of Psychiatry, LMU Munich). The general design is based on the previously published EXTassay approach, where cis-regulatory sensors drive the expression of unique molecular barcodes, so called EXTs (Botvinnik et al., 2010). In brief, cis-regulatory sensors consist either of clustered transcription factor binding sites and response elements upstream of the minimal adenoviral major late promoter (minMLP) (termed cis-element-based sensors), or of 1-1.5 kb long endogenous promoter fragments which harbor the transcription start site (termed promoter-based sensors). The corresponding sequences were either synthesized by Genscript or PCR amplified from genomic DNA. Cloning was done using the MultiSite Gateway pro plus kit (Invitrogen) according to the manufacturer's instructions. Thereby, three insert fragments were cloned into the pAAVspace_DEST_luc2_WPRE (V825) vector by recombination. For cis-elementbased sensor, the three fragments consist of the (i) clustered cis-element, (ii) the minMLP, 
and (iii) the molecular barcode. In case of a promoter-based sensor, the three fragments are (i) a stuffer sequence, (ii) the promoter, and (iii) the molecular barcode (see figure 10A). All constructs were verified by restriction digest and sequencing of the insert.

\section{Cell culture}

E15.5 wt primary cortical mouse neurons were seeded in 6-well plates at 0.5 million cells/well. Cultures were infected on DIV5 with the AAV cis-regulatory sensor library with 2500 AAV GCs per cell. A duplicate sample was silenced by treatment with $1 \mu \mathrm{M}$ TTX and $100 \mu \mathrm{M}$ APV on DIV12. On DIV14, a duplicate sample was harvested as an untreated reference. The remaining samples were stimulated with $50 \mu \mathrm{M} \mathrm{BIC,} 100 \mu \mathrm{M}$ 4-AP, $100 \mu \mathrm{M}$ glycine, and $1 \mu \mathrm{M}$ strychnine for 2,4 , and 8 hours in duplicates. All samples were finally harvested using Qiazol reagent. The RNA was purified using the Zymo Direct-zol RNA MiniPrep kit. Subsequently, the purified RNA was treated with TurboDNase to digest residual AAV genomes and cleaned up by a second column purification using the Zymo Direct-zol RNA MiniPrep kit according to the manufacturer's instructions.

The purified RNA was reverse transcribed as followed:

Total RNA

Random nonamer primer $(120 \mu \mathrm{M})$

dNTPs (10 mM each)
$4.5 \mu \mathrm{l}$

$1 \mu \mathrm{l}$

$0.5 \mu \mathrm{l}$

5 minutes at $65^{\circ} \mathrm{C}$, followed by 1 minute on ice. Then add per reaction:

$\begin{array}{ll}\text { 5x First-strand reaction buffer } & 2 \mu \mathrm{l} \\ \text { DTT }(0.1 \mathrm{M}) & 1 \mu \mathrm{l} \\ \text { SuperScript III RT } & 1 \mu \mathrm{l}\end{array}$

Incubate first at $50^{\circ} \mathrm{C}$ for 30 minutes, followed by 15 minutes at $70^{\circ} \mathrm{C}$.

Following the cDNA synthesis, barcodes were amplified by PCR with Dec1/2 primers (250 $\mathrm{nM}$ each) using HotStar Taq plus DNA polymerase (Qiagen). The cDNA input was $1 \mu \mathrm{l}$ of a $1 / 10$ dilution into a $20 \mu \mathrm{l} \mathrm{PCR}$ reaction. In addition, an external barcode mix was added to the PCR reaction, which can be used for calibration between samples. The PCR was performed with an annealing temperature of $59^{\circ} \mathrm{C}$ and 30 cycles. The PCR product was verified by agarose gel-electrophoresis.

In a second PCR, the adapter sequences for lon Torrent sequencing were fused to the barcodes. The forward code primer contained the lon-A adapter sequence required for lon Torrent sequencing and a 12bp code sequence for multiplexing of samples in a single sequencing run. The reverse primer contained the lon-P1 adapter sequence required for lon 
Torrent sequencing. The PCR was done using HotStar Taq plus DNA polymerase (Qiagen). The PCR product was verified by agarose gel-electrophoresis. The final PCR products were pooled and purified using the NucleoSpin Gel and PCR Clean-up kit (Macherey\&Nagel).

Barcode libraries were sequenced on an Ion Torrent PGM sequencer using the 318 chip. The sequencing service was provided by Stefanie Behrens and Dr. Sabrina Galinski (Molecular Neurobiology, Department of Psychiatry, LMU Munich). All template preparations and enrichments were done according to the manufacturer's protocols for the Ion PGM Template OT2 $200 \mathrm{kit}$. Sequencing was done according to the manufacturer's protocols for the Ion PGM Sequencing 200 v2 kit. Processing of the raw data was done in collaboration with Dr. Sven Wichert (Molecular Neurobiology, Department of Psychiatry, LMU Munich) using custom shell and $\mathrm{R}$ scripts. First, raw reads were split into individual samples using the 12 bp code and subsequently mapped to a reference barcode library using a local BLAST. Thereby, reads were counted. Next, read counts were normalized to total read numbers per sample. Normalized read counts were standardized to read counts of a sensor which contains only the minMLP, but no cis-regulatory element. Finally, data was presented as a heatmap of log2-transformed fold changes relative to the untreated reference sample.

\subsection{Luciferase assays}

Firefly luciferase reporter gene assays were performed during the development and validation of the PATHscreener and PATHscreener2.0 vector and for the validation of individual candidates from the screen.

\section{Multiplate luciferase assays}

For multiplate assays, cells were seeded into 96-well plates and either transfected (for cell lines) or infected (for primary neurons). Cells were transfected using Lipofectamine2000 (Invitrogen) according to the manufacturer's instructions or infection by an E-SAREluciferase containing AAV. Infection was done with 500-1000 AAV GCs per cell. The assay always compares an unstimulated response with a stimulated response.

Validation of individual shRNAs was done with primary neurons in 24-well plates. Neurons were infected with AAV PATHscreener vectors either expressing an shRNA or a nontargeting control RNA. Cultures were treated the same way as the screening samples (BIC/4-AP vs. TTX/APV). At the end of the assay, cells were lysed using Passive lysis buffer (Promega). The luciferase activity was measured by a Mithras LB 940 Microplate Reader (Berthold Technologies) and the software MicroWin2000. 
Live cell luciferase activity was measured using the LumiCycle 32 apparatus. Therefore, primary neurons were seeded onto $3.5 \mathrm{~cm}$ culture dishes. The medium was supplemented with 1/1000 luciferin (Promega).

\subsection{Protein detection by immunocytochemistry}

Immunocytochemistry was used to quantify synapses in maturing primary neuron cultures and to verify the expression of CRISPR-Cas9 components. Therefore, primary neurons were grown on glass coverslips. The entire staining procedure was performed at room temperature. First, 1 vol. of $4 \%$ paraformaldehyde (PFA) was added to the cell culture medium for fixation and incubated for 15 minutes. Next, the neurons were washed three times with PBS. Neurons were permeabilized with $0.1 \%$ Triton-X-100 in PBS for 1 minute, followed by three washes with PBS. Subsequently, ICC blocking solution was applied for 30 minutes. The neurons were then incubated with the primary antibody in $0.1 \%$ ICC blocking solution for one hour. Afterwards, neurons were washed three times for 5 minutes each with PBS. The secondary fluorescently labeled antibody was applied for one hour in $0.1 \%$ ICC blocking solution. Finally, the coverslips were washed three times with PBS for 5 minutes each, briefly rinsed in $\mathrm{dH}_{2} \mathrm{O}$, and mounted onto glass slides using Prolong Gold Antifade reagent (containing DAPI for nuclei staining).

\section{Synapse staining}

Primary antibodies: mouse anti-MAP2 (1:200); rabbit anti-synaptophysin (1:250)

Secondary antibodies: Alexa488 anti-mouse (1:500); Alexa647 anti-rabbit (1:500)

\section{CRISPR-Cas9 staining}

Primary antibodies: mouse anti-FLAG-M2 (1:100); rat anti-HA (1:100)

Secondary antibodies: Alexa488 anti-mouse; Cy3 anti-rat (both 1:500)

Image acquisition and analysis

Images were acquired using a Zeiss Observer Z1 microscope in combination with the Zeiss Zen 2012 software. For the synapse quantification, images were analyzed with Fiji ImageJ using the 'Find Maxima' function. The synapse count was normalized to the mean intensity of the MAP2 staining. 


\subsection{Protein detection by western blot}

Western blotting was used to verify the expression of the CRISPR-Cas9 components dCas9 (HA-tagged) and MS2-p65-VP64 (FLAG-tagged) in HEK293 cells. Therefore, HEK293 cells were seeded into 6-well plates (0.5 million/well) and transfected either with V1785 or V1787 using Lipofectamine2000 (Invitrogen) according to the manufacturer's protocol. The western blot was performed by Barbara Meisel (Molecular Neurobiology, Department of Psychiatry, LMU Munich). In brief, cells were washed once with PBS on ice and harvested by lysis in 1\% Triton-X protein lysis buffer (containing protease inhibitors). The lysate was transferred into a reaction tube and centrifuged at $13,000 \mathrm{rpm}$ for 10 minutes at $4^{\circ} \mathrm{C}$ to clear cell debris. Next, the protein samples were mixed with Loading/reducing buffer and proteins were denatured at $70^{\circ} \mathrm{C}$ for 10 minutes. Proteins were separated on a Mini-PROTEAN TGX, 4-15\% gel (Biorad) prior to blotting onto a PVDF transfer membrane Hybond P (GE Healthcare). Afterwards, the blot was first incubated with blocking solution (5\% milk powder in TBS-T) followed by the primary antibody and the secondary HRP-conjugated antibody. Imaging of the blots was done using the ECL ChemoCam Imager (INTAS).

Primary antibodies: rat anti-HA (1:1000), mouse anti-FLAG-M2 (1:5000), mouse anti-GAPDH (loading control, 1:1000)

Secondary antibodies: anti-rat-HRP (1:5000), anti-mouse-HRP (1:5000)

\subsection{RNA detection by reverse transcription and qRT-PCR}

Relative mRNA quantification was used to determine the knockdown of an shRNA or the activation of gene expression by CRISPRa. In both cases, total RNA was isolated using the Zymo Direct-zol RNA MiniPrep kit according to the manufacturer's instructions, including an on-column DNA digest. The first-strand cDNA synthesis was done using SuperScript III reverse transcriptase kit (Invitrogen). qRT-PCR assay primers were designed using the Roche Universal Probe Library assay design center. qRT-PCRs were performed on a Qiagen Rotor-Gene cycler using the 2x Rotogene SYBR green Master Mix. Rpl13a was used as a reference gene. The relative quantification was done with the Qiagen Rotogene software using the $\Delta \Delta \mathrm{Ct}$-method (Schmittgen and Livak, 2008). 


\subsection{Design and cloning of the PATHscreener library}

Insert preparation

The shRNA library template for the insert has been the Decipher Mouse Module 1 shRNA library by Cellecta. The insert was prepared by two consecutive PCRs in order to add the synthetic polyA signal (SpA) and the random barcode (BC35).

PCR\#1

Decipher shRNA library $(10 \mathrm{ng} / \mu \mathrm{l}) \quad 1 \mu \mathrm{l}$

SpA oligo (2 $\mathrm{nM})$

Dec rev primer $(10 \mu \mathrm{M})^{*} \quad 0.5 \mu \mathrm{l}$

hU6 fwd primer $(10 \mu \mathrm{M})^{*} \quad 0.5 \mu \mathrm{l}$

$\mathrm{dH} 2 \mathrm{O} \quad 7 \mu \mathrm{l}$

2x PWO Master Mix $10 \mu \mathrm{l}$

* add after first 5 cycles

PCR parameters: $95^{\circ} \mathrm{C} 2 \mathrm{~min}, 95^{\circ} \mathrm{C} 20 \mathrm{sec}, 59^{\circ} \mathrm{C} 20 \mathrm{sec}, 72^{\circ} \mathrm{C} 30 \mathrm{sec}\left(5\right.$ cycles), $95^{\circ} \mathrm{C} 20$ sec, $55^{\circ} \mathrm{C} 20 \mathrm{sec}, 72^{\circ} \mathrm{C} 30 \mathrm{sec}(20$ cycles $)$

The PCR product has been purified using the NucleoSpin Gel and PCR Clean-up kit (Macherey\&Nagel) and validated by $2 \%$ agarose gel-electrophoresis.

PCR\#2

Product PCR\#1 (2e+09 molecules/ $\mu \mathrm{l}) \quad 1 \mu \mathrm{l}$

BC35 oligo $(4 \mathrm{e}+09$ molecules/ $\mu \mathrm{l}) \quad 1 \mu \mathrm{l}$

BC35 rev primer $(10 \mu \mathrm{M}) \quad 0.5 \mu \mathrm{l}$

hU6 fwd primer $(10 \mu \mathrm{M}) \quad 0.5 \mu \mathrm{l}$

$\mathrm{dH} 2 \mathrm{O} \quad 7 \mu \mathrm{l}$

2x PWO Master Mix $10 \mu \mathrm{l}$

PCR parameters: $95^{\circ} \mathrm{C} 2 \mathrm{~min}, 95^{\circ} \mathrm{C} 20 \mathrm{sec}, 55^{\circ} \mathrm{C} 20 \mathrm{sec}, 72^{\circ} \mathrm{C} 30 \mathrm{sec}(10 \mathrm{cycles}$ )

The PCR product has been purified using the NucleoSpin Gel and PCR Clean-up kit (Macherey\&Nagel) and validated by $2 \%$ agarose gel-electrophoresis.

The purified product of PCR\#2 was digested with BamHI and Clal for 2 hours at $37^{\circ} \mathrm{C}$ and subsequently purified using the NucleoSpin Gel and PCR Clean-up kit (Macherey\&Nagel). The final insert concentration was determined using the Picodrop spectrophotometer. 
The empty PATHscreener vector $(\mathrm{V} 1301,10 \mu \mathrm{g})$ was digested with BamHI and Clal for 6 hours at $37^{\circ} \mathrm{C}$ and purified by $1 \%$ agarose gel-electrophoresis and the NucleoSpin Gel and PCR Clean-up kit (Macherey\&Nagel). Additionally, the linearized vector was purified by phenol/chloroform/ethanol extraction. Therefore, 1 vol. of phenol was added, the solution was mixed by shaking for 20 times and centrifuged at full speed for 5 minutes. The upper layer was transferred to a new tube and $100 \mu \mathrm{l}$ TE buffer was added to the phenol phase for re-extraction. Afterwards the two upper layers were pooled and the procedure was repeated with 1 vol. of chloroform. Finally, the linearized vector DNA was precipitated by adding 1/10 vol. of $3 \mathrm{M}$ sodium-acetate $\mathrm{pH} 5.2$ and 2.5 vol. 100\% ethanol. For precipitation it was vortexed and kept at $-20^{\circ} \mathrm{C}$ for 1 hour. Subsequently, the DNA was pelleted by full speed centrifugation at $4^{\circ} \mathrm{C}$ for 10 minutes. The supernatant was removed and the pellet was washed once with cold $70 \%$ ethanol. After this washing step the pellet was air-dried for 5 minutes and resuspended in $20 \mu \mathrm{ITE}$ buffer.

\section{Ligation}

Ligation was done with $500 \mathrm{ng}$ linearized vector and a vector:insert ratio of 1:3. Prior to the ligation, the mix of vector and insert was heated up to $55^{\circ} \mathrm{C}$ for 2 minutes and then snapfrozen at $-20^{\circ} \mathrm{C}$ for 10 minutes followed by thawing on ice. Next, $1 \mu \mathrm{l} 10 \mathrm{x}$ T4 ligase buffer (NEB) and $1.4 \mu \mathrm{l}$ T4 DNA ligase (NEB) was added (final vol. $10 \mu \mathrm{l}$ ) and the ligation reaction was incubated at $16^{\circ} \mathrm{C}$ over night. Before transformation, $10 \mu \mathrm{l} \mathrm{H} 2 \mathrm{O}$ were added to the reaction in order to reduce the salt concentration and the T4 ligase was heat inactivated at $65^{\circ} \mathrm{C}$ for 10 minutes.

\section{Transformation}

Transformation was done by electroporation. MegaX Dh10b E.coli (20 $\mu \mathrm{l})$ were added to $2 \mu \mathrm{l}$ ligation reaction. The mix was gently pipetted up and down and transferred to an ice-cold electroporation cuvette. Immediately the electroporation was done the bacteria were recovered by adding $1 \mathrm{ml}$ pre-warmed Recovery medium (included with MegaX Dh10b E.coli). The electroporated bacteria were incubated at $37^{\circ} \mathrm{C}$ for 1 hour with shaking. A dilution series from 1-10 $\mu \mathrm{l}$ was spread onto $10 \mathrm{~cm}$ LB-agar (ampicillin) plates for colony counting the next day. Twelve mini cultures were inoculated with individual colonies in order to verify the cloning. Colony number per volume was calculated and the volume for $\sim 10,000$ colonies was spread onto a $15 \mathrm{~cm}$ LB-agar (ampicillin) plate. In total 3 plates were inoculated in order to get a maximum number of $\sim 30,000$ different barcodes within the library. The next day, colonies from all 3 plates were collected and plasmid DNA was purified using the NucleoBond PC100 Midiprep Kit (Macherey-Nagel). 
In order to assign the barcode sequence to the shRNA the region encoding the barcode and the shRNA was amplified by PCR using primers with lon Torrent sequencing adapters (PGM_A_IXcode3_AFA_s fwd primer / PGM_trP1_hU6_as rev primer). The PCR product has a size of $345 \mathrm{bp}$ and was sequenced on an lon Torrent PGM sequencer using the 318 chip. The sequencing service was provided by Stefanie Behrens and Dr. Sabrina Galinski (Molecular Neurobiology, Department of Psychiatry, LMU Munich). All template preparations and enrichments were done according to the manufacturer's protocols for the Ion PGM Template OT2 400 Kit. Sequencing was done according to the manufacturer's protocols for Ion PGM Hi-Q Sequencing Kit.

The analysis was done using a custom $\mathrm{R}$ script which included the following steps: Raw reads were filtered by length ( $>231 \mathrm{bp}$ ) in order to cover the barcode sequence and the shRNA antisense strand. Using the Biostrings function matchLRPatterns() filtered raw reads were scanned for the barcode (left pattern) and the $8 \mathrm{bp}$ adjacent to the shRNA antisense strand (right pattern). 5 mismatches were allowed in the left pattern without indels and 3 mismatches in the right pattern with indels. Thereby, barcode sequences and shRNA antisense strand sequences were extracted. Next, shRNA antisense strand sequences were aligned by BLAST to the Cellecta shRNA library reference list. At this stage, a matrix with a barcode ID, the barcode sequence, the shRNA sequence, and the shRNA ID, and the Refseq ID of the shRNA target was created. This matrix was finally filtered for barcodes that were coupled to the same shRNA in more than $80 \%$ of the cases. The final barcode library was aligned to itself using a local megablast with varying parameters to determine optimal megablast stringency for barcode discrimination during the screen. These were an e-value threshold of $1 \mathrm{e}-10$ and a word-size of 12 .

\subsection{Pooled RNAi screen in primary neurons}

\section{Cell culture}

Primary cortical neuron cultures were prepared from E15.5 wt mice. Neurons from 16-24 embryos were pooled per screen. Per sample, 10 million or 5 million cells were seeded onto PLL-coated $15 \mathrm{~cm}$ or $10 \mathrm{~cm}$ tissue culture dishes, respectively. In parallel, PLL-coated 3.5 $\mathrm{cm}$ dishes were seeded with 0.5 million cells to record the treatments in the Lumicycler. 2-4 replicate cultures were prepared per treatment condition. Seeding was done in Neurobasal medium supplemented with 5\% FBS, 2\% B27, and 1\% GlutaMax. On DIV1, the medium was replaced by Neurobasal medium supplemented with 2\% B27 and $1 \%$ GlutaMax (NeuroCulture medium; $20 \mathrm{ml}$ per $15 \mathrm{~cm}$ dish, $10 \mathrm{ml}$ per $10 \mathrm{~cm}$ dish). On DIV6, cultures were 
infected with the AAV-PATHscreener library at an AAV particle to cell ratio of 1000:1. Simultaneously, cultures were fed by adding pre-warmed NeuroCulture medium ( $5 \mathrm{ml}$ per 15 $\mathrm{cm}$ dish, $2.5 \mathrm{ml}$ per $10 \mathrm{~cm}$ dish, and $0.5 \mathrm{ml}$ per $3.5 \mathrm{~cm}$ dish). On DIV10, all cultures were fed with pre-warmed NeuroCulture medium $(5 \mathrm{ml}$ per $15 \mathrm{~cm}$ dish, $2.5 \mathrm{ml}$ per $10 \mathrm{~cm}$ dish, $0.5 \mathrm{ml}$ per $3.5 \mathrm{~cm}$ dish) and half of the cultures were in addition treated with $1 \mu \mathrm{M} \mathrm{TTX}$ and $100 \mu \mathrm{M}$ APV to silence neuronal activity. The culture for real-time luciferase recordings were in addition supplemented with the firefly luciferase substrate luciferin and the recording using the Lumicycler was started. On DIV12, the cultures which have not been silenced were stimulated with a cocktail containing $50 \mu \mathrm{M}$ BIC, $100 \mu \mathrm{M}$ 4-AP, $100 \mu \mathrm{M}$ glycine, $1 \mu \mathrm{M}$ strychnine for 4 hours.

\section{Harvest}

For cell harvest of silenced and stimulated cultures the culture medium was aspirated and cells were rinsed once with cold PBS. Subsequently, QIAzol cell lysis reagent was applied for lysis ( $5 \mathrm{ml}$ for $15 \mathrm{~cm}$ dishes, $2.5 \mathrm{ml}$ for $10 \mathrm{~cm}$ dishes). Cell lysate was scraped from the dishes and transferred into a $15 \mathrm{ml}$ tube. The lysate was kept at $-80^{\circ} \mathrm{C}$ until RNA isolation.

\section{Total RNA isolation}

Lysates were thawed at room temperature and cell debris was pelleted by centrifugation at $4000 \mathrm{rpm}$ for 5 minutes. The supernatant was transferred into a new $15 \mathrm{ml}$ tube and the total RNA was isolated using the Zymo Direct-zol RNA MiniPrep kit according to the manufactures instructions with the following modifications. The lysate from 10 million cells was split onto 2 RNA purification columns in order to not exceed the RNA binding capacity of a column. Elution was done in $50 \mu \mathrm{l}$ Rase-free $\mathrm{H} 2 \mathrm{O}$ per column and the two eluates from 10 million cells were pooled afterwards.

The total RNA was quantified using a Picodrop spectrophotometer. $\sim 60 \mu \mathrm{g}$ or $\sim 30 \mu \mathrm{g}$ total RNA was obtained from 10 million or 5 million cells, respectively.

In order to digest traces of co-isolated AAV genomes, total RNA was treated with TurboDNase for 30 minutes at $37^{\circ} \mathrm{C}$. For $\sim 60 \mu \mathrm{g}$ total RNA this was done in a volume of 300 $\mu \mathrm{l}$ using $6 \mu \mathrm{l}$ TurboDNase. With less total RNA input the reaction was scaled down accordingly. The DNase-digested total RNA was subsequently cleaned-up by adding 1 vol. of $100 \%$ ethanol and using the Zymo Direct-zol RNA MiniPrep Kit for purification. One column was used per sample. Elution in $25 \mu \mathrm{l}$ RNase-free H2O. Total RNA was again quantified using a Picodrop spectrophotometer. 
cDNA synthesis

The first-strand cDNA synthesis was done using the Invitrogen SuperScript III reverse transcriptase. The entire total RNA was reverse transcribed in multiple $20 \mu \mathrm{l}$ reactions containing $5 \mu \mathrm{g}$ total RNA each and using oligo(dT) primer. The reaction protocol was as followed:

Total RNA

Oligo(dT) primer $(50 \mu \mathrm{M})$

dNTPs (10 mM each)

$\mathrm{H} 2 \mathrm{O}$

$$
\begin{aligned}
& 5 \mu \mathrm{g} \\
& 1 \mu \mathrm{l} \\
& 1 \mu \mathrm{l} \\
& \text { Up to } 13 \mu \mathrm{l}
\end{aligned}
$$

5 minutes at $65^{\circ} \mathrm{C}$, followed by 1 minute on ice. Then add per reaction:

$\begin{array}{ll}\text { 5x First-strand reaction buffer } & 4 \mu \mathrm{l} \\ \text { DTT }(0.1 \mathrm{M}) & 1 \mu \mathrm{l} \\ \text { H2O } & 1 \mu \mathrm{l} \\ \text { SuperScript III RT } & 1 \mu \mathrm{l}\end{array}$

Incubate first at $50^{\circ} \mathrm{C}$ for 30 minutes, followed by 15 minutes at $70^{\circ} \mathrm{C}$.

Barcode quantification by $q R T-P C R$

In order to validate the sensor induction during the screen, RNA barcode expression was quantified relative to Rpl13a expression or absolute using a plasmid standard with $1 \mathrm{e}+02$ copies $-1 e+05$ copies/ $\mu$ l. Primer pairs were qDec1.2/qDec2.2 for the barcode and the plasmid standard and qRT-PCR primer for Rpl13a. Analysis was done using the Qiagen Rotor-Gene Software with the $\Delta \Delta$ Ct-method for relative quantification.

$\begin{array}{ll}\text { 2x RotorGene SYBRgreen PCR Master Mix } & 5 \mu \mathrm{l} \\ \text { Fwd primer }(10 \mu \mathrm{M}) & 1 \mu \mathrm{l} \\ \text { Rev primer }(10 \mu \mathrm{M}) & 1 \mu \mathrm{l} \\ \text { cDNA (pre-diluted 1:100) } & 3 \mu \mathrm{l}\end{array}$

Default qRT-PCR cycling parameters.

Dec PCR

The 'Dec PCR' amplifies the barcode from the cDNA sample. Prior to the 'Dec PCR', the entire cDNA was purified using the Macherey\&Nagel PCR clean-up kit und eluted with $20 \mu \mathrm{l}$ elution buffer. Per sample $100 \mu \mathrm{l}$ reactions were prepared, split into $2 \times 50 \mu \mathrm{l}$ reactions for PCR and pooled again afterwards. 


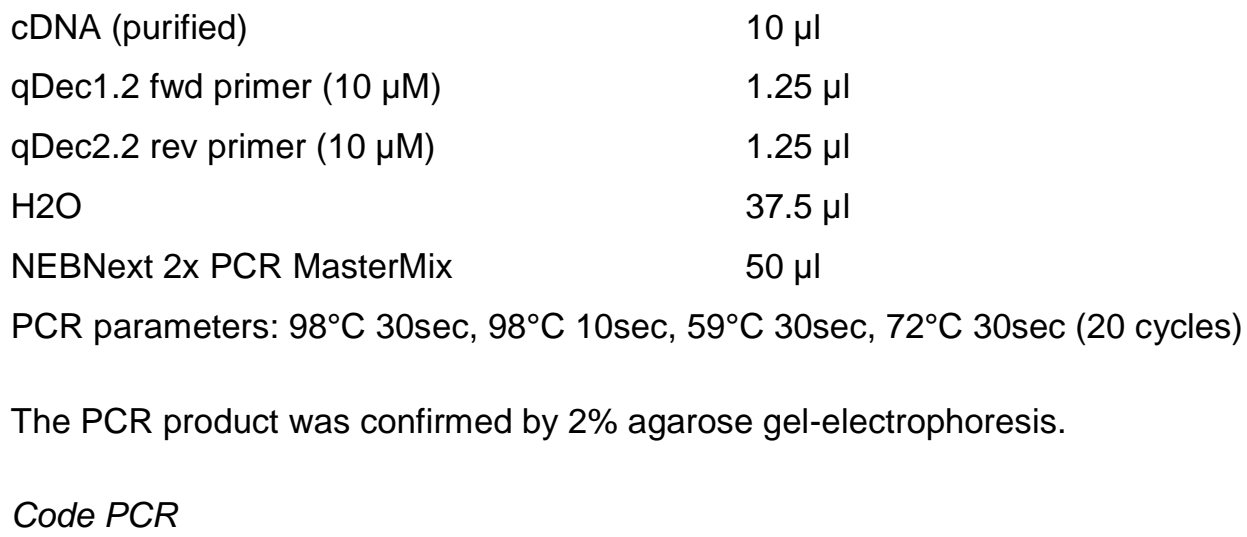

The 'Code PCR' fuses sample specific 12bp code sequences to the 'Dec PCR' product in order to pool samples for next-generation sequencing. The forward code primer contains the Ion-A adapter sequence required for lon Torrent sequencing and the 12bp code sequence. The reverse primer contains the Ion-P1 adapter sequence required for lon Torrent sequencing. Code PCR reaction per screen sample:

$\begin{array}{ll}\text { Dec PCR product (pre-diluted 1:10) } & 5 \mu \mathrm{l} \\ \text { Code fwd primer }(10 \mu \mathrm{M}) & 0.625 \mu \mathrm{l} \\ \text { Code rev primer }(10 \mu \mathrm{M}) & 0.625 \mu \mathrm{l} \\ \text { H2O } & 18.75 \mu \mathrm{l} \\ \text { NEBNext 2x PCR MasterMix } & 25 \mu \mathrm{l}\end{array}$

PCR parameters: $98^{\circ} \mathrm{C} 30 \mathrm{sec}, 98^{\circ} \mathrm{C} 10 \mathrm{sec}, 58^{\circ} \mathrm{C} 30 \mathrm{sec}, 72 \mathrm{C} 30 \mathrm{sec}$ (10 cycles)

The PCR product was confirmed by $2 \%$ agarose gel-electrophoresis.

20-40 $\mu \mathrm{l}$ per sample were pooled subsequently and purified using the NucleoSpin Gel and PCR Clean-up kit (Macherey\&Nagel).

Next-generation sequencing of barcodes

Barcode libraries were sequenced on an Ion Torrent Proton sequencer using the PI chip. The sequencing service was provided by Stefanie Behrens and Dr. Sabrina Galinski (Molecular Neurobiology, Department of Psychiatry, LMU Munich). All template preparations and enrichments were done according to the manufacturer's protocols for the lon $\mathrm{PI}$ Template OT2 200 v3 kit. Sequencing was done according to the manufacturer's protocols for the Ion PI Sequencing 200 v3 kit. One PI chip delivered on average 100 million raw reads.

Processing of the raw data was done in collaboration with Dr. Sven Wichert (Molecular Neurobiology, Department of Psychiatry, LMU Munich) using custom shell and R scripts. First, raw reads were split into individual samples using the 12 bp code and subsequently mapped to a reference barcode library using a local BLAST. Thereby, reads were counted 
and assigned to shRNAs and gene targets. Next, read counts were normalized to total read numbers per sample. If multiple barcodes are assigned to the same shRNA, corresponding read counts were summed. To control the correlation between replicates, similarities between all samples were estimated using pair-wise Pearson correlation coefficient and plotted as a heatmap with hierarchical clustering. Analysis was then continued by enhanced Z-score ranking or using the DESeq2 $\mathrm{R}$ package (Love et al., 2014).

For the enhanced Z-score analysis, normalized read counts of replicates were collapsed to mean count values and log2 transformed. Log2 ratios were calculated between stimulated and silenced samples and normalized to enhanced Z-scores. In order to collapse to gene level, the barcode/shRNA with the strongest effect towards the positive- (for negative regulators) or negative direction (for positive regulators) was selected to represent a certain gene.

The DESeq2 package allows testing for differential expression of a gene or in this case of a barcode. Therefore, normalized read count data with all replicates for the stimulated and silenced conditions was first processed using the DESeqDataSetFromMatrix() function. Next, data was analyzed using the DESeq() function which includes the Wald test for differential expression and correction by multiple testing using the Benjamini-Hochberg method (Benjamini and Hochberg, 1995).

\section{KEGG and Reactome pathway analysis}

Pathway analysis of the top positive regulators from the enhanced Z-score ranking was done using the KEGG database and the Reactome database (Fabregat et al., 2016; Ogata et al., 1998). The KEGG analysis was done via the WEBGESTALT homepage (http://bioinfo.vanderbilt.edu/webgestalt/) and the Reactome analysis was done using the analysis function of the Reactome Pathway Browser (http://www.reactome.org/PathwayBrowser/).

\subsection{Cloning individual shRNAs and sgRNAs}

In order to validate screen results, selected shRNAs from the library were individually cloned into the AAV E-SARE PATHscreener vector (V1301). Pairs of oligonucleotides were synthesized by Eurofins with Agel and EcoRl-compatible overhangs and annealed as followed. In $50 \mu \mathrm{l}$ volume, $2.5 \mu \mathrm{g}$ of each oligonucleotide were mixed together with $5 \mu \mathrm{l} 10 \mathrm{x}$ T4 DNA ligase buffer (NEB). The mix was incubated at $90^{\circ} \mathrm{C}$ for 2 minutes and then slowly cooled down to room temperature to allow annealing of the two shRNA strands. The annealed oligonucleotides were diluted to $5 \mathrm{ng} / \mu \mathrm{l}$ and ligated into the linearized AAV ESARE PATHscreener vector. 
shRNA oligonucleotide pairs were designed as followed:

Forward: 5'-CCGG-shRNA forward sequence-3'

Reverse: 5'-AATT-shRNA reverse sequence-3'

Cloning of sgRNAs into the linearized PATHscreener2.0 vector was done using the same protocol as for shRNAs. For linearization, the PATHscreener2.0 vector was digested with Bbsl, creating overhangs for the ligation.

sgRNA oligonucleotide pairs were designed as followed:

Forward: 5'-ACCG-sgRNA target sequence-3'

Reverse: 5'-AAAC-sgRNA reverse target sequence-3' 


\section{Results}

\subsection{General considerations for a pooled RNAi screen in neurons}

Until now the majority of pooled functional genetic screens (RNAi or CRISPR-Cas9) have been performed in oncology (Diehl et al., 2014; Shalem et al., 2015). This has an obvious impact on existing protocols and plasmid libraries. In oncology, the general goal is to screen for modifiers of cell proliferation/survival. To achieve the desired segregation of 'hitcells' from 'non-hit-cells' in terms of cell numbers within the total cell population, long cultivation periods over multiple passages are required. This implies the use of lentiviral libraries (shRNA or sgRNA) to generate stable integrations in the genome of the mitotic cell line of interest (Rubinson et al., 2003; Stewart et al., 2003). The long selection procedure in order to get a strong phenotype finally allows isolating the genomic DNA and counting the number of integrations per shRNA by next-generation sequencing. An alternative approach involves the use of reporter cell lines and flow cytometry as a strategy to segregate different phenotypes at the endpoint of the screen and to focus the readout to cellular functions other than proliferation/survival (Parnas et al., 2015). This strategy is closest to the technology presented within the following chapters.

The aim to perform a pooled RNAi screen in a post-mitotic cell type, such as primary neurons, required certain adjustments to the classical protocols. Standard cell culture protocols for primary mouse neurons allow cultivation for not more than a few weeks and viability usually declines after two weeks in culture. Thus, the time window to produce a strong cell survival phenotype is relatively short. Nevertheless, to screen for modifiers of cell survival would be of great interest in terms of neurodegenerative diseases. Therefore, it has been initially tested how genomic DNA and total RNA abundance changes in response to cytotoxic stress within a primary neuron population. Cultures where treated with the electron transport chain inhibitor rotenone at different concentrations for 7 days and cell number, genomic DNA and total RNA was quantified. Surprisingly, the total nuclei count was only modestly reduced even at high rotenone concentrations $(>100 \mathrm{nM})$, although quantification of viable cells, by filtering out all pyknotic nuclei, revealed the toxicity of rotenone with an IC50 of $14 \mathrm{nM}$ (Figure 9A). This already indicated that an analysis based on a DNA barcode readout might lack the required sensitivity in a cell viability paradigm. In accordance to the cell count, it has been observed that only at rotenone concentrations above 100nM a reduction of the genomic DNA content by $\sim 40 \%$ could be detected (IC50 $40 \mathrm{nM}$ ). However, a more sensitive change in total RNA content with an IC50 (15 nM) comparable to the live cell count and a higher dynamic range compared to genomic DNA was measured (Figure 9B). Taken together, this indicated that without long-term culturing including cell passaging, a segregation of viable and non-viable cell pools based on a DNA reporter is not feasible. 
Furthermore, RNA-based barcode readouts seem to be preferable in the case of primary neurons.

A

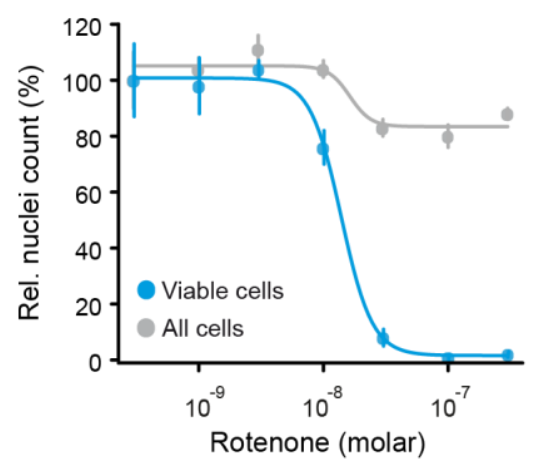

B

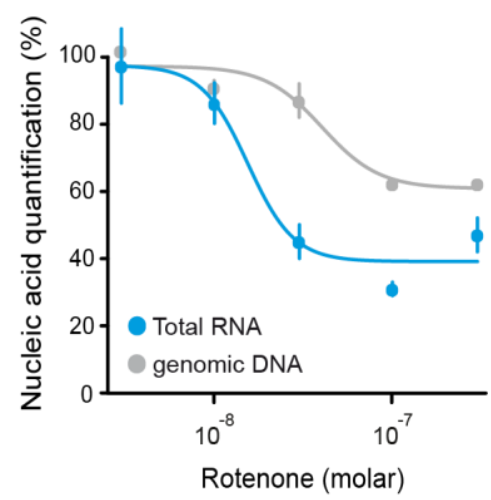

Figure 9: Cellular RNA content is a more sensitive indicator of neuronal viability in the dish than DNA.

A. Rotenone killing-curve. Primary neurons were treated with the indicated Rotenone concentrations from DIV7 till DIV14. Cells were counted by nuclear Hoechst stain. Either all Hoechst positive nuclei were quantified (All cells) or only non-pyknotic nuclei to discriminate viable from non-viable cells (Viable cells) ( $n=3+$-sem). B. Quantification of total RNA and genomic DNA content from primary neuron cultures in response to metabolic stress by Rotenone treatment $(n=2$ + -sem).

A second modification to current protocols was the viral system used to transduce primary neurons for a pooled RNAi screen. Primary neurons are post-mitotic cells and thus integration of the shRNA library is not needed for stable long-term transgene expression. Since the readout does not require stable barcode integration as well, it has been decided to use recombinant Adeno-associated virus (AAV). This has several practical and experimental advantages. AAVs require less safety precautions and are structurally more robust than lentiviruses (Bouard et al., 2009). More importantly, however, is that AAVs produced with certain capsid serotypes (e.g. serotype 1 and 2) have a natural tropism for neuronal cells and do not trigger a cellular immune response (McCown, 2005). Within the cell the AAV genome preferentially persists extrachromosomally (Nakai et al., 2001). This might reduce the risk of integration locus effects on shRNA and barcode expression and eliminates the possibility that coding or regulatory regions in the genome are destroyed due to integration (Moiani et al., 2012).

However, the first attempt to use a pooled shRNA library in order to screen for modifiers of neuronal survival highlighted that the assay transfer from oncology to neuroscience is not trivial. The cytotoxic stress paradigm did not led to a strong quantitative shift of the shRNAcoupled barcode abundances within the neuron population after one week of treatment (data 
not shown). This emphasized that a highly sensitive readout is absolutely necessary for successful pooled loss-of-function screenings in primary neurons. Therefore, it has been hypothesized that a more promising readout option would be the use of a reporter which monitors a cellular state (e.g. pathway activity). This might not only improve sensitivity and robustness of the screen but would provide proof-of-principle for an interference assays that leverages a sensor-assisted approach (since sensors can be flexibly exchanged) to dissect signaling networks principally in any genetically amenable cell type with pathway specificity and at a genomic scale.

\subsection{A reporter for neuronal activity}

The response to synaptic stimulation involves a cascade of cellular events which propagate the signal from the synapse to the nucleus. Stimulation of excitatory synapses causes an influx of calcium through NMDA receptors and L-type voltage-gated calcium channels (Dolmetsch, 2003). The main signaling routes to the nucleus involve calcium-dependent activation of calcium/calmodulin-dependent protein kinases and of adenylate cyclases (Greer and Greenberg, 2008). This results in activation of CaMK4 and PKA. Activated PKA and CaMK4 phosphorylate a set of pre-existing transcription factors (e.g. CREB, MEF2) (Flavell et al., 2006; Hardingham et al., 1997). These transcription factors induce expression of activity-dependent immediate early genes (e.g. Fos, Arc, Npas4). This activity-dependent gene expression program has been studied in great depth using RNA-seq and ChIP-seq technologies (Kim et al., 2010; Malik et al., 2014; Zhang et al., 2009). However, already during the pre-omics era activity-dependent genes have been studied and the transcription factor Fos was one of the first identified immediate early gene that is induced by neuronal excitation (Greenberg et al., 1985; Morgan et al., 1987; Sheng and Greenberg, 1990). Since then, the Fos gene product or the Fos promoter ( 1 kb upstream sequence of the transcription start site) coupled to a reporter (e.g. GFP) have been extensively used to label and identify activated neurons (Garner et al., 2012; Schilling et al., 1991). While the induction of the Fos promoter is sufficient to discriminate cell populations of activated versus silent neurons, its dynamic range is relatively small and thus probably not sensitive enough as a quantitative readout for a high-throughput RNAi screen.

In order to identify a genetic sensor with a wide dynamic range upon synaptic stimulation, a multiplexed cis-regulatory sensor assay was performed. Each sensor within this assay either consists of a clustered transcription factor binding sites (cis-element) coupled to a minimal adenoviral major late promoter (minMLP) or a $\sim 1 \mathrm{~kb}$ promoter fragment (Figure 10A). During the assay sensors are driving the expression of unique molecular RNA barcodes in response to cellular signaling events. Barcode transcripts are finally isolated and decoded by NGS in 
order to measure sensor activities (Figure 10B). For the identification of a synaptic activity sensor, primary cortical neurons were infected with the AAV pool of 70 sensor vectors and the sensor activities were measured at DIV14 under neuronal silencing conditions, basal activity (i.e. untreated), and synaptic stimulation. Silencing of neuronal activity has been achieved through the application of the voltage-gated sodium channel inhibitor tetrodotoxin (TTX) in combination with the NMDAR antagonist D-(-)-2-Amino-5-phosphonopentanoic acid (APV) (McLennan, 1981; Narahashi et al., 1966). Neuronal activity of the culture has been evoked by blocking inhibitory synaptic transmission using the $\mathrm{GABA}_{\mathrm{A}}$ receptor antagonist bicuculline $(B I C)$. In all experiments, BIC has been supplemented with the potassium channel blocker 4-Aminopyridine (4-AP), the NMDAR co-agonist glycine, and the glycine receptor antagonist strychnine (Curtis et al., 1970; Meves and Pichon, 1975). This stimulation cocktail is referred to as BIC/4-AP. Multiple sensors qualified as synaptic activity reporters as they showed reduced activity in silenced neurons and increased barcode expression after synaptic stimulation (Figure 10C). The largest dynamic range between synaptic silencing and stimulation was measured for a sensor consisting of an enhancer from the murine Arc promoter, called synaptic activity-responsive element (SARE) (Kawashima et al., 2009). The SARE sensor outperformed classical neuronal activity reporters such as the endogenous promoters of Fos and Egr1 (Figure 10C). 


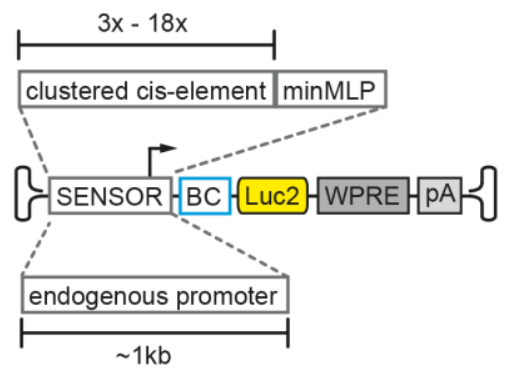

B

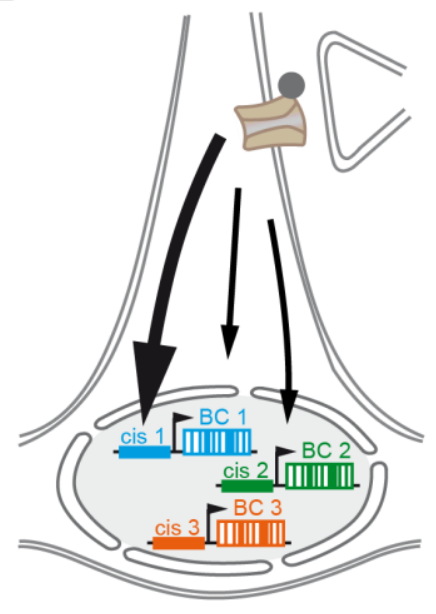

C

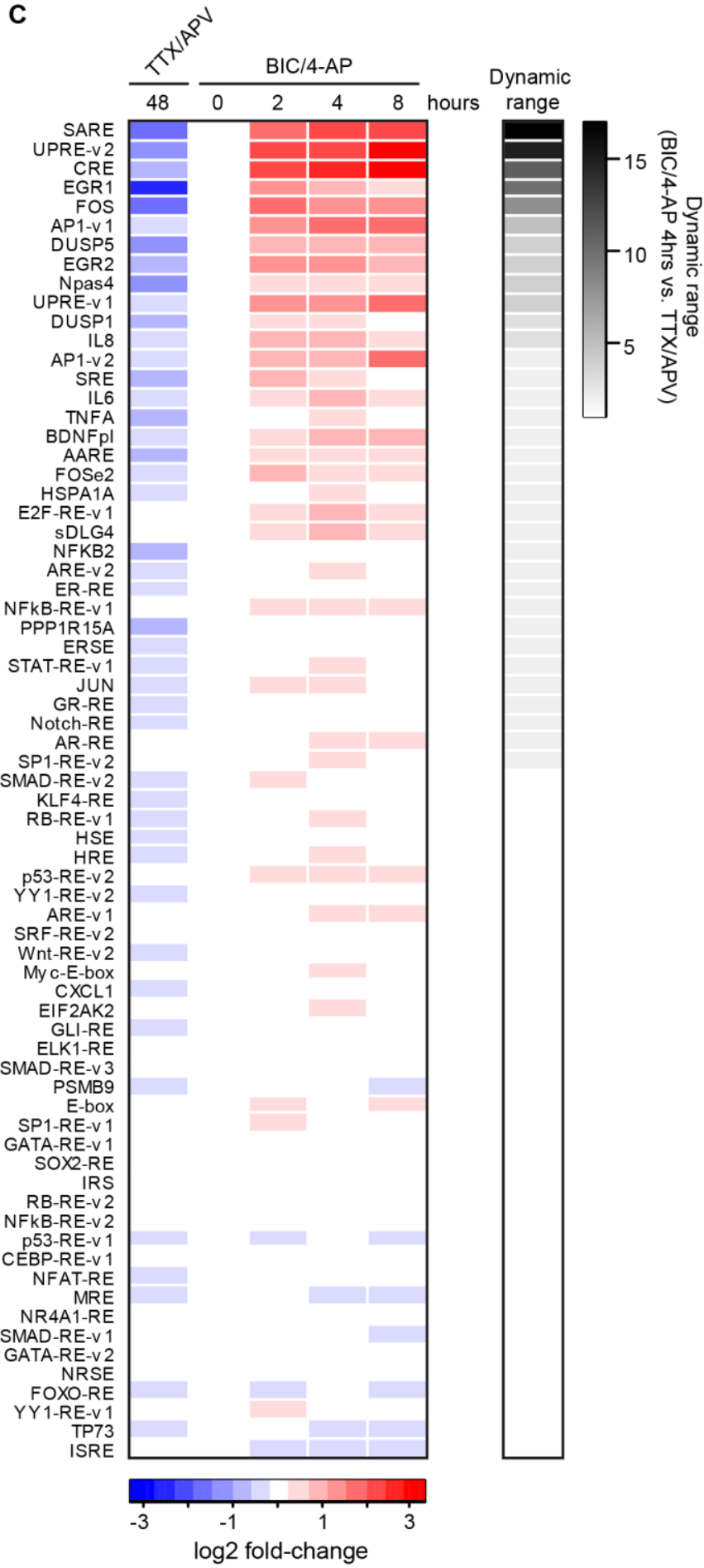

Figure 10: Multiplexed cis-regulatory sensor assay in response to neuronal silencing and synaptic stimulation.

A. Schematic map of the cis-regulatory sensor vector. The barcode (BC) and the firefly luciferase (luc2) are driven by clustered transcription factor binding site (cis-elements) or endogenous promoters. B. Illustration of the assay design. Neuronal cultures are infected by a pool of sensor vectors packaged into AAV particles and the sensor response is measured by NGS of the barcode pool. C. Heatmap of 70 sensor responses to TTX/APV or BIC/4-AP at the indicated hours of treatment (log2 fold changes). Sensors are ranked by dynamic range of BIC/4-AP (4hrs) vs. TTX/APV (right heatmap). 
The SARE enhancer is a $\sim 100 \mathrm{bp}$ sequence localized $>6 \mathrm{~kb}$ upstream of the Arc gene and contains binding sites for three activity-dependent transcription factors (CREB, MEF2 and SRF) (Figure 11). The Arc gene is expressed in response to neuronal activity and ARC protein is enriched at the post-synaptic density of dendritic spines and exerts functions during synaptic plasticity via regulation of AMPA receptor endocytosis (Chowdhury et al., 2006; Plath et al., 2006; Shepherd et al., 2006). Kawashima and colleagues have originally identified the SARE enhancer and characterized its sensitivity to synaptic stimulation (Kawashima et al., 2009). A genome-wide bioinformatics search for SARE-like sequences has revealed that this arrangement of transcription factor binding sites is not unique for the Arc promoter, but is found in promoter regions of many other neuronal activity-dependent genes (Rodríguez-Tornos et al., 2013).

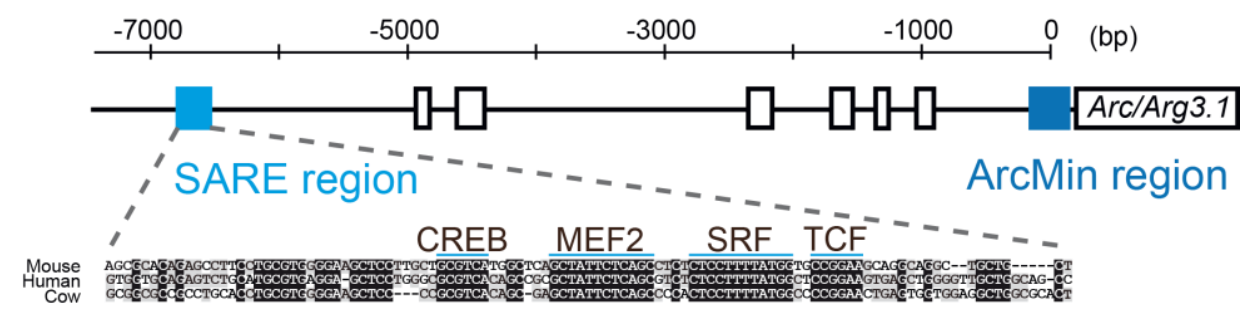

Figure 11: Genomic architecture of the murine Arc promoter.

Top, the SARE and ArcMin regions are indicated by blue boxes. Evolutionarily conserved genomic regions are represented by white boxes. Bottom, sequence alignment of the SARE region between mouse, human and cow. Sequences of high conservation are highlighted in black boxes. Binding sites for CREB, MEF2 and SRF/TCF are indicated. Modified from(Kawashima et al., 2013).

During the development of the sensor pool for the cis-regulatory assay, the SARE sensor has been optimized for highest signal-to-noise ratio by clustering of multiple SARE repeats in front of a 420bp minimal Arc promoter (ArcMin), similar to work by Kawashima and colleagues (Kawashima et al., 2013). Sensors with one, three, four, five, and six SARE repetitions were tested for a maximal dynamic range in the neuronal cell line SH-SY5Y upon stimulation with PMA (phorbol-12-myristat-13-acetat). A cluster of four SARE repetitions gave highest fold inductions and was therefore used in all subsequent experiments (Figure 12A). This composite promoter is hereafter named enhanced SARE (E-SARE) sensor, in line with the nomenclature used by Kawashima and colleagues, although their construct contains five SARE repetitions. Next, the E-SARE sensor was further characterized in primary cortical neurons. Longitudinal recordings of E-SARE-driven luciferase activity from untreated, maturing primary neurons revealed that the baseline E-SARE activity recapitulates the course of increasing synaptogenesis, as determined by staining the presynaptic marker synaptophysin in maturing cultures (Figure 12B). The kinetics also match the staging by Baj 
and colleagues shown in figure 4 (Baj et al., 2014). Finally, the E-SARE sensor not only responds to neuronal silencing (TTX/APV) and synaptic stimulation (BIC/4-AP), but can also be induced by brain-derived neurotrophic factor (BDNF) which is a well-established stimulus with functions in neuronal differentiation and plasticity (Figure 12C) (Park and Poo, 2013). The optimized and validated E-SARE sensor has been subsequently used for developing the pooled RNAi screen in primary neurons.

A
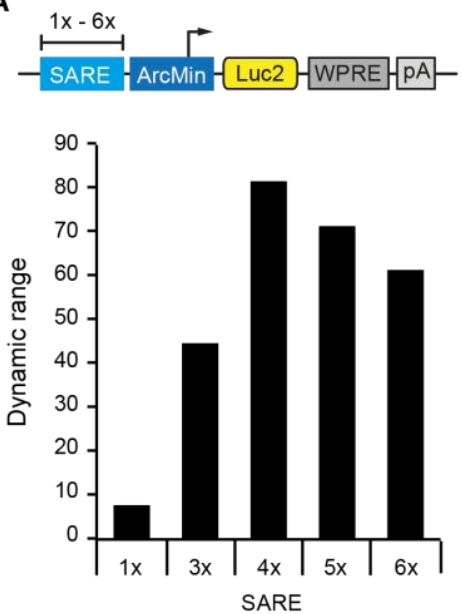

B
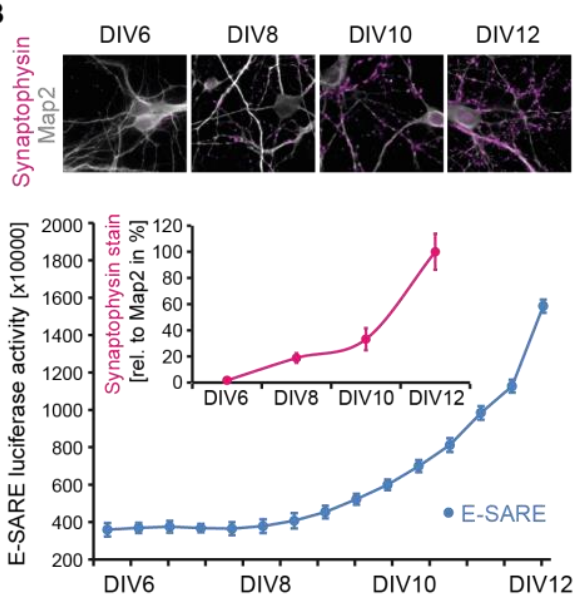

C

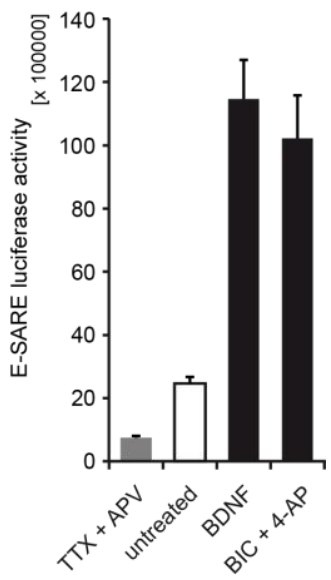

Figure 12: Design and characterization of the artificial E-SARE sensor.

A. Top, Schematic map of the sensor-luciferase vector with clustered SARE enhancers. Bottom, Comparison of luciferase activity of clustered SARE reporters after stimulation with PMA in SHSY5Y cells $(n=6)$. A cluster of four SARE is hereafter called enhanced SARE (E-SARE) sensor. B. Longitudinal measurement of basal E-SARE activity in maturing primary neurons from DIV6-12 $(\mathrm{n}=3+$-sem). Inset, Synapse quantification by staining of the presynaptic marker synaptophysin $(n=3+-s e m)$. Top, representative images of primary neurons (DIV6-12) stained with antibodies against synaptophysin and MAP2. C. E-SARE activity in primary neurons upon silencing (TTX/APV), basal activity (untreated), and stimulation (BDNF, BIC/4-AP) at DIV14 ( $\mathrm{n}=6+$ +-sd).

\subsection{Vector design for sensor-based RNAi screenings}

\subsubsection{The principle of the screening approach}

The presented study aimed to combine a pooled shRNA screen with a sensitive and robust genetic sensor. This sensor should consist of an optimized synthetic promoter (as described in section 5.2) which regulates the expression of a RNA barcode upon pathway activation. The use of a barcoded genetic sensor has two major advantages over currently used readout options for pooled interference screens (RNAi or CRISPR-Cas9): (1) It allows to measure cellular phenotypes other than proliferation/survival which is the case if only the bare shRNA pool complexity is analyzed. (2) It is independent of cell sorting based on a 
fluorescent reporter and therefore a direct quantitative readout. In order to achieve the combined approach of a pooled RNAi screen with a pathway activity readout, two libraries need to become one, the shRNA library and the sensor-coupled molecular barcode library. Each barcode within this library is controlled by the same genetic sensor and codes for an shRNA that is expressed from the same AAV vector (Figure 13A). The corresponding vector is hereafter named PATHscreener vector.

The screen itself is based on the simplified hypothesis that a stimulus (e.g. receptor agonist) triggers a signaling cascade which induces the barcoded sensor and that interference (by shRNAs) within this pathway leads to an altered sensor induction compared to shRNAs that are not targeting the pathway (Figure 13B). Sensor activities are finally measured by counting of the corresponding barcodes using NGS. The screen is based on the assumption that each cell is only infected once in order to avoid cross-contamination of multiple shRNAs and barcodes. In addition, we consider it as extremely unlikely that even at infection rates slightly greater than one per cell, by chance two shRNAs act in a synergistic or opposing fashion and may therefore compromise the screen. 
A

PATHscreener vector

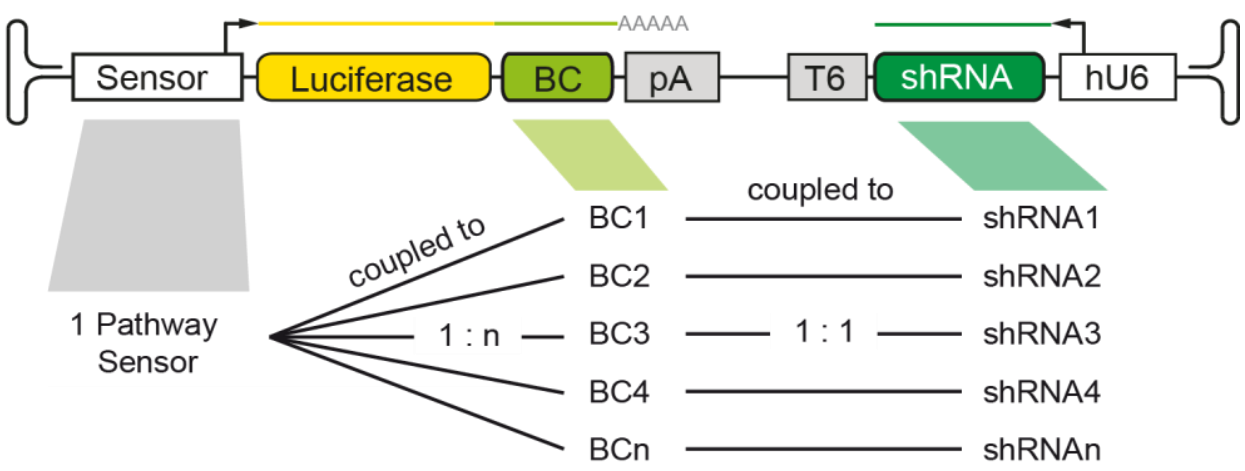

B

shRNA w/o effect on pathway:

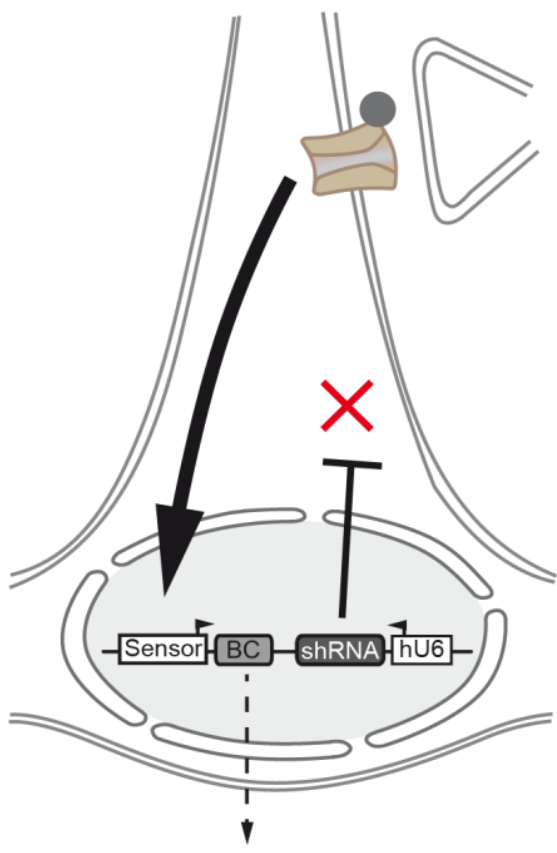

Strong barcode induction rel. to unstimulated ref.

shRNA with effect on pathway:

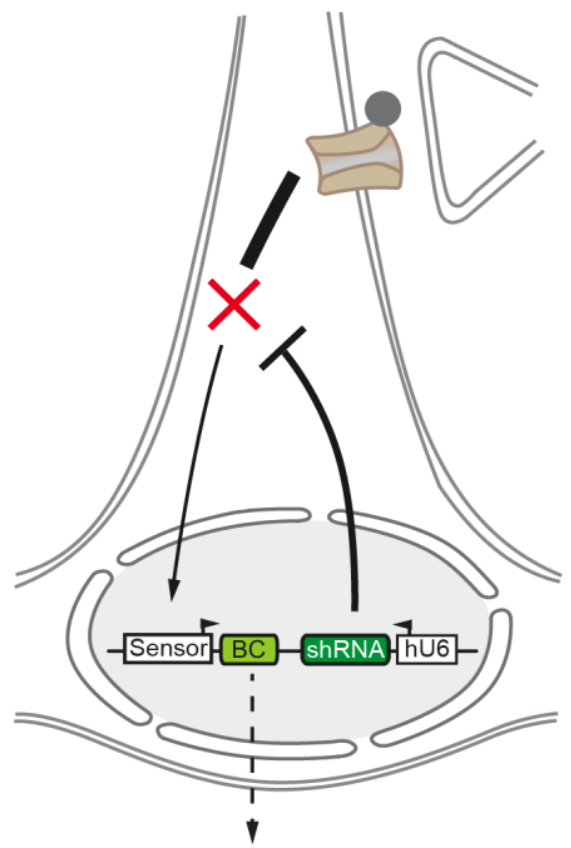

Weak barcode induction rel. to unstimulated ref.

Figure 13: Principle of the sensor-based genetic interference screen.

A Map of the AAV PATHscreener vector. The connection within the library between sensor, barcodes and shRNAs is indicated below. B Graphical summary of the working hypothesis for the sensor-coupled RNAi screen. Interference independent of a given pathway addressed with a corresponding sensor does not affect the readout (left), whereas shRNAs targeting the signaling cascade alter sensor activity (right).

\subsubsection{The shRNA expression cassette}

Soon after the discovery of the post-transcriptional regulatory mechanism called RNA interference, its use as a research tool for gene expression manipulation has rapidly evolved to a gold standard technique (Mohr et al., 2014). The expression of shRNAs by a strong 
RNA polymerase III promoter (e.g. human U6 promoter) is the most commonly used method for stable RNAi. However, at the time when this project was initiated protocols and constructs have been published which allow shRNA expression from DNA polymerase II promoters (Li et al., 2007; Liu et al., 2010; Zuber et al., 2011). One advantage is the ability to use cell type specific promoters (please refer to section 2.4. for a detailed discussion on RNAi tools). The success and depth of a genetic interference screen relies on the efficiency of the genetic manipulation. As knockdown efficiency cannot be increased by multiple infections per cell during a pooled screen it is necessary to express optimized shRNAs from a strong promoter. In order to compare the impact of different promoters on RNAi efficiency, knockdown of a luciferase reporter by five different shRNAs driven by the hU6 promoter (hU6p or the DNA polymerase II promoters of synapsin-1 (Syn1p) or neuron-specific enolase (NSEp) were determined (Figure 14A). Overall, the hU6p-driven shRNAs showed superior knockdown efficiencies. In particular when the shRNA efficiency is suboptimal, the hU6 promoter demonstrates its impressive strength to boost the knockdown (Figure 14A, shRNA\#4). Moreover, only the hU6 promoter-mediated RNAi achieved knockdown efficiencies above $90 \%$ in this test. Hence, the hU6 promoter was selected to drive the expression of the shRNA library for screening irrespective of possible limitation given the absence of cell type specificity.

In order to transduce primary neurons with the sensor-coupled shRNA library, AAV is the vector of choice as it has a natural tropism for neurons and does not evoke any cellular immune response (McCown, 2005). Whether the expression of a transgene or an shRNA is stable in primary neurons over time, was determined by infecting at DIV1 with an AAV which expresses GFP and an shRNA against Tcf4 and analyzing GFP fluorescence and Tcf4 mRNA abundance at various time points until DIV14 (Figure 14B and C). Knockdown of the Tcf4 mRNA was first detectable at four days post-infection which is also the time needed for AAV (serotype 1/2) infection until reaching its maximum. The knockdown was increasing and remained stable until DIV14. In accordance to this observation are the results obtained from GFP imaging. GFP expression is first visible at two days post-infection and increases until it stays stable over the entire time course (Figure 14C). This indicates that the AAV system is well suited for the screening approach and that the risk of losing AAV genomes or epigenetic silencing of promoters over time appears to be probably negligible. 
A

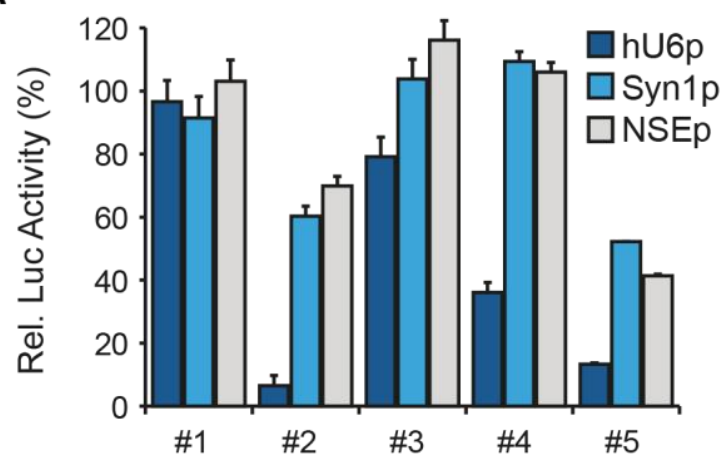

shRNA against firefly luciferase
B

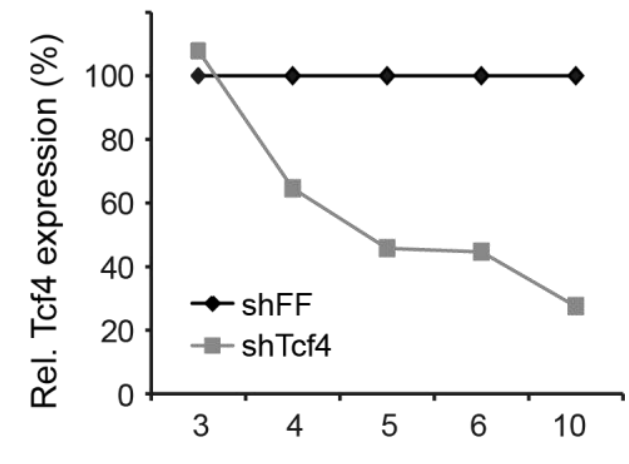

Days post-infection

C

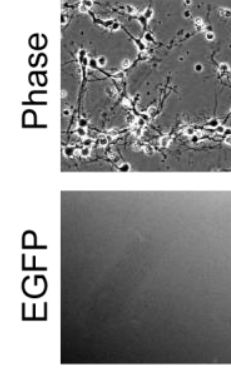

1

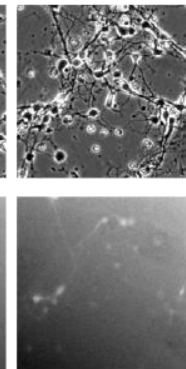

2

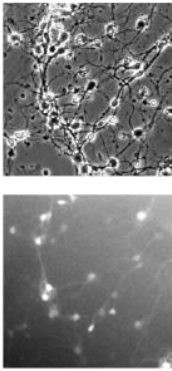

3
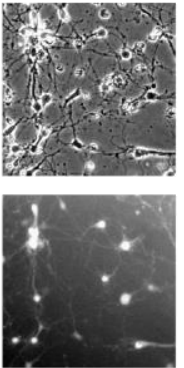

4

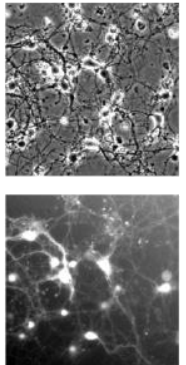

5

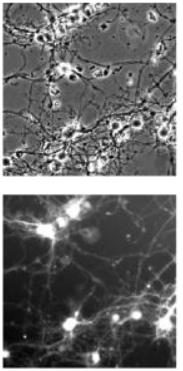

6

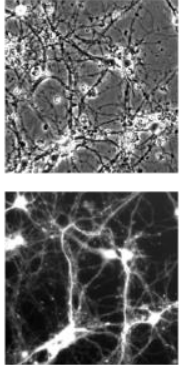

10

Days post-infection

Figure 14: Efficient mRNA knockdown by hU6 promoter-driven shRNAs in neuronal cells.

A. Efficacy of five shRNAs, targeting firefly luciferase, driven by the hU6p, Syn1p, or NSEp promoter. shRNA expression plasmids were co-transfected with a luciferase reporter plasmid into PC12 cells $(n=6,+-s d)$. B. Quantification of Tcf 4 mRNA expression in primary neurons infected with AAVs for shRNA expression against Tcf4 or firefly luciferase as a non-targeting control on DIV1. Tcf4 mRNA was quantified at the indicated days post-infection. C. Longitudinal imaging of GFP expression in primary neurons infected on DIV1 with AAV-Syn1p-GFP (serotype 1/2).

In summary, the following milestones for the development of a sensor-coupled pooled RNAi screen were accomplished: (1) The E-SARE sensor gives a strong and robust induction in response to neuronal activity. (2) The hU6 promoter is the promoter of choice for efficient shRNA-mediated interference with gene expression. (3) AAVs are suitable vectors for stable transgene and shRNA expression in primary neurons. Next, the screening vector, as illustrated in figure 13A, needed to be generated and tested for functionality. 


\subsubsection{Combining sensor and shRNA expression}

In the previous chapters the general design of the screening vector and independent validations of the barcoded E-SARE sensor and shRNA expression cassette have been presented. Next, it has been crucial to determine how both parts function in parallel and in close proximity if cloned into the same AAV backbone, which has a capacity of $\sim 4.8 \mathrm{~kb}$. This distance between the two promoters is relatively small, compared to genomic scales of promoter regions and one major concern was that the sensor might be biased due to regulatory elements of the hU6 promoter (Das et al., 1988). To address this issue the ESARE induction upon PMA stimulation was determined in a luciferase assay with the complete AAV PATHscreener vector (containing E-SARE sensor and hU6p) or vectors where either the E-SARE sensor (w/o sensor) or the hU6p-shRNA cassette (w/o hU6pshRNA) were deleted. Comparison of the complete PATHscreener vector with the hU6pshRNA deletion vector ruled out the initial concerns and showed that the sensor is not compromised by the hU6 promoter (Figure 15A). Both vectors generated similar luciferase activities at baseline as well as upon stimulation by PMA.

An essentially important requirement for the pooled RNAi screen is that the sensor shows uniform inductions across different constructs with variable barcode and ShRNA sequences. To test this condition 10 constructs with different barcode and shRNA sequences were cloned. E-SARE induction for each clone was tested by PMA stimulation in rat PC12 cells to minimize the risk of a true shRNA effect on the sensor activity. Overall the inductions across all clones were similar and the variance was considered acceptable (Figure 15B). None of the clones deviated more than three median absolute deviations (MAD) from the median, which is a frequently used hit criterion in high-throughput screens (Birmingham et al., 2009).

The two expression cassettes, sensor and shRNA, are directed towards each other which is defined by the library cloning procedure (described in chapter 5.4). Hence, the barcode is located downstream of the shRNA cassette, only separated by a synthetic poly-adenylation signal ( $\mathrm{SpA}$ ) and the T6 terminator (six thymidines) (see Figure 13A). Early research on the transcriptional termination from DNA polymerase III promoters has revealed that the termination is not always efficient and that run-through transcripts can occur (Campbell and Setzer, 1992). In case of the screening vector this might cause barcode transcription from the hU6 promoter (Figure 15C, dashed orange line). Since expression from the hU6 promoter is strong even slight termination inefficiency could lead to a severe contamination problem during sequencing if those barcode sequences are transcribed into cDNA. To avoid such a contamination it might be sufficient to use an oligo(dT) primer during first-strand cDNA synthesis. This primer only hybridizes with poly(A)-tails of transcripts expressed by the sensor. In addition, a comparison of random primers with oligo(dT) primers for cDNA 
synthesis indicated that the oligo(dT) primer is superior in this set-up and the data show that transcriptional run-through by DNA polymerase III is not a problem (Figure 15C).

A

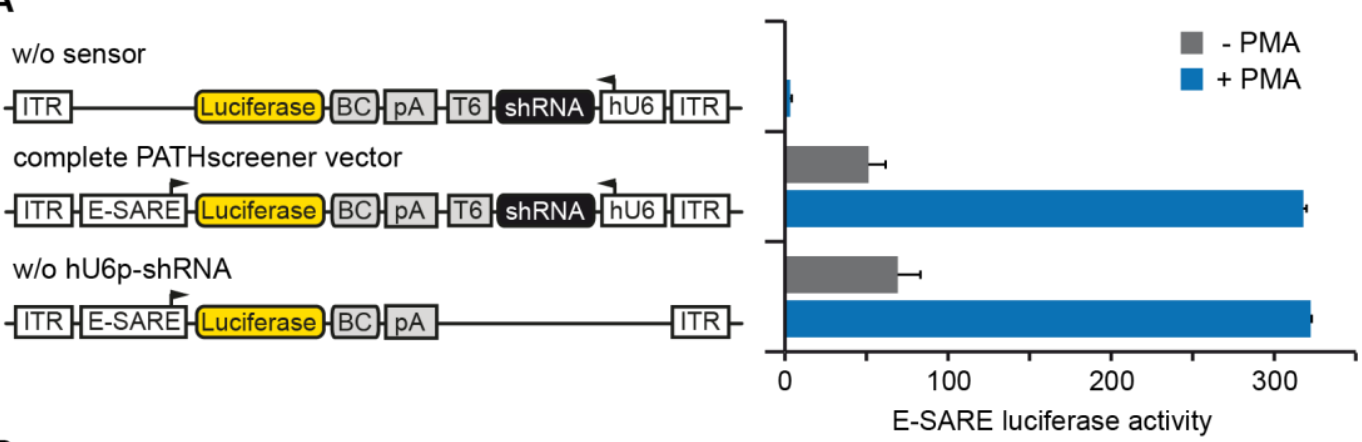

B

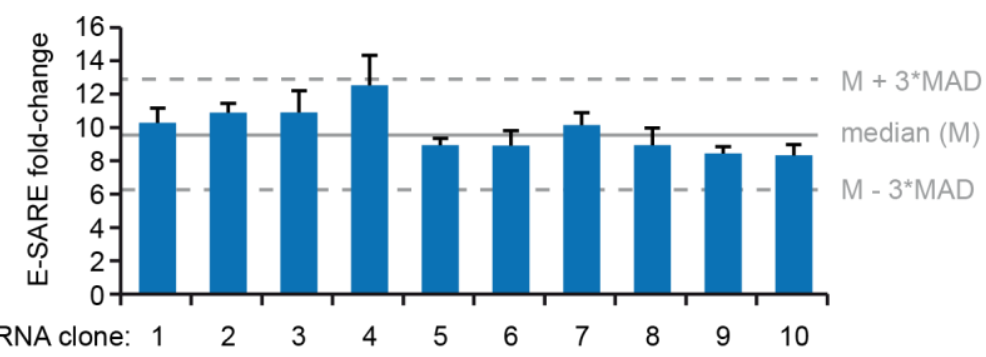

C

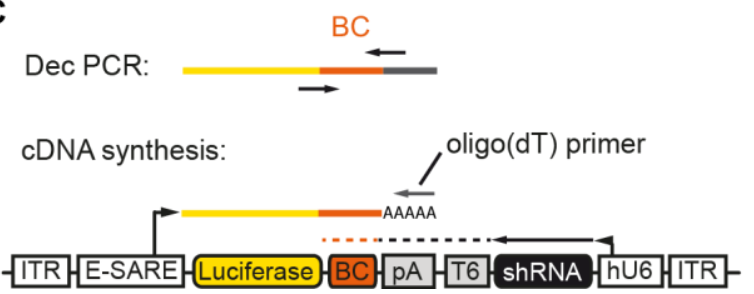

M w/o sensor complete w/o hU6p NTC

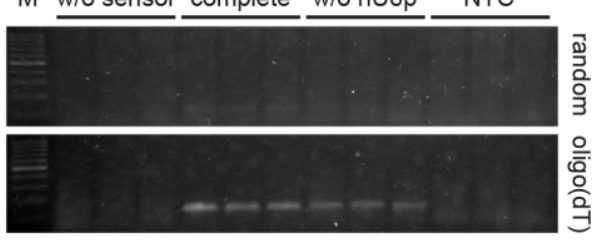

Figure 15: Functional validation of the PATHscreener vector.

A. Validation of an unbiased sensor response in the dual-expression PATHscreener vector. PC12 cells were transfected with the complete vector or a vector with an E-SARE sensor deletion or hU6p-shRNA deletion. Luciferase activity was measured for unstimulated and PMA-stimulated samples $(n=6+/-s d)$. B. PC12 cells were transfected with the PATHscreener vector expressing 10 different random shRNAs. E-SARE-luciferase fold change upon PMA stimulation does not deviate more the three median absolute deviations (MAD) from the median $(n=6+-s d)$. C. Left, schematic of the vector with transcripts expressed by the sensor and by the hU6 promoter. The dashed line indicates DNA polymerase III run-through transcript. The oligo(dT) primer for cDNA synthesis is indicated as well as primer binding sites for barcode amplification at the decoding (Dec) PCR. Right, PC12 cells were transfected with the vectors shown in A and purified RNA was transcribed to CDNA either with random primers or oligo(dT) primers. A prominent Dec PCR barcode product is only detectable with cDNA transcribed using oligo(dT) primers. NTC, non-template control. 


\subsection{Library cloning strategy}

A high quality shRNA library is the basis for successful pooled RNAi screenings. Libraries can be custom-made by high-throughput synthesis of shRNA oligonucleotides or purchased from various suppliers. Libraries can be either genome-wide or focused regarding specific groups of genes. Parameters affecting the decision between a genome-wide or focused approach are the biological question of the screen and the required cell number in order to get robust results. Common sense is that a cell number to shRNA complexity ratio of 2001000:1 is required for robust screenings. For primary cells, cell numbers are often limited which argues for a focused library format. The aim of this study was to screen for regulators of neuronal excitation and synapse-to-nucleus signaling, hence the library should focus on signaling pathway genes in general. Such a library has been generated by Cellecta as part of the Decipher project (http://www.decipherproject.net/). The Decipher Mouse Module 1 (MM1) shRNA library covers 4625 genes that were selected based on expert-curated pathway databases like KEGG and Reactome, the CSHL Cancer 1000 List, the Cancer Genome Atlas, FDA drug targets and $\mathrm{MeSH}$. With nearly 5000 gene targets the library is perfectly sized for pooled RNAi screenings in primary neurons. The general design of the shRNA stem and loop region has been thoroughly optimized by Cellecta for highest knockdown efficiencies. To make use of a high quality shRNA library design we decided to use the Decipher MM1 shRNA library and to develop a cloning strategy for repurposing of commercial shRNA libraries in a different context. The cloning strategy requires the following steps (Figure 16A): (1) PCR amplification of the hU6p-shRNA library cassette from the original shRNA plasmid library and simultaneous fusion of a minimal SpA (Levitt et al., 1989). The SpA will finally belong to the sensor cassette. (2) Fusion of the shRNA library PCR product with an oligonucleotide library containing random barcode sequences and amplification by PCR. (3) Large-scale ligation of the shRNA-barcode library insert into the sensor containing AAV backbone. (4) Sequencing of the vector region spanning the shRNA and the barcode in order to assign barcode sequences to shRNAs/gene targets (Figure 16B). The Ion Torrent PGM which was used for deep-sequencing of the final library is able to sequence fragments up to $\sim 400 \mathrm{bp}$. This implies that shRNA and barcode must be in close proximity and as a consequence both expression cassettes are facing towards each other and the SpA was selected as one of the smallest available poly-adenylation signals (Levitt et al., 1989). During library cloning, 12 individual clones were isolated and analyzed by restriction digest and Sanger sequencing. All clones passed the restriction digest, only one clone contained a mutated insert and each clone had a unique shRNA and barcode sequence. This confirmed that the protocol is delivering reliable cloning products. The cloned library contains $\sim 25,000$ unique barcodes which code for $\sim 13,000$ different shRNAs covering $\sim 4500$ genes (Table 1). Hence, after library repurposing $\sim 97 \%$ of target genes are covered. An shRNA complexity of $\sim 13,000$ allows to perform a screen in a $15 \mathrm{~cm}$ cell culture dish with 
10 million cells. With an infection rate of $60 \%, \sim 500$ cells would be infected per shRNA on average. The barcode to shRNA ratio of $\sim 2: 1$ results from a 2:1 ratio between barcode oligo and hU6p-shRNA-SpA fragment during PCR\#2. This provides evidence that the cloning strategy by PCR is well tunable. A feature which makes this cloning strategy superior to protocols which are commonly used for library cloning in MPRA projects, for example. Those protocols require a barcoded backbone vector library into which an enhancer library is cloned. This usually results in higher numbers of different barcodes per enhancer. The generated E-SARE-shRNA library was successfully packaged into AAV particles (serotype $1 / 2$ ) and subsequently used for screenings in primary neuron cultures.

A

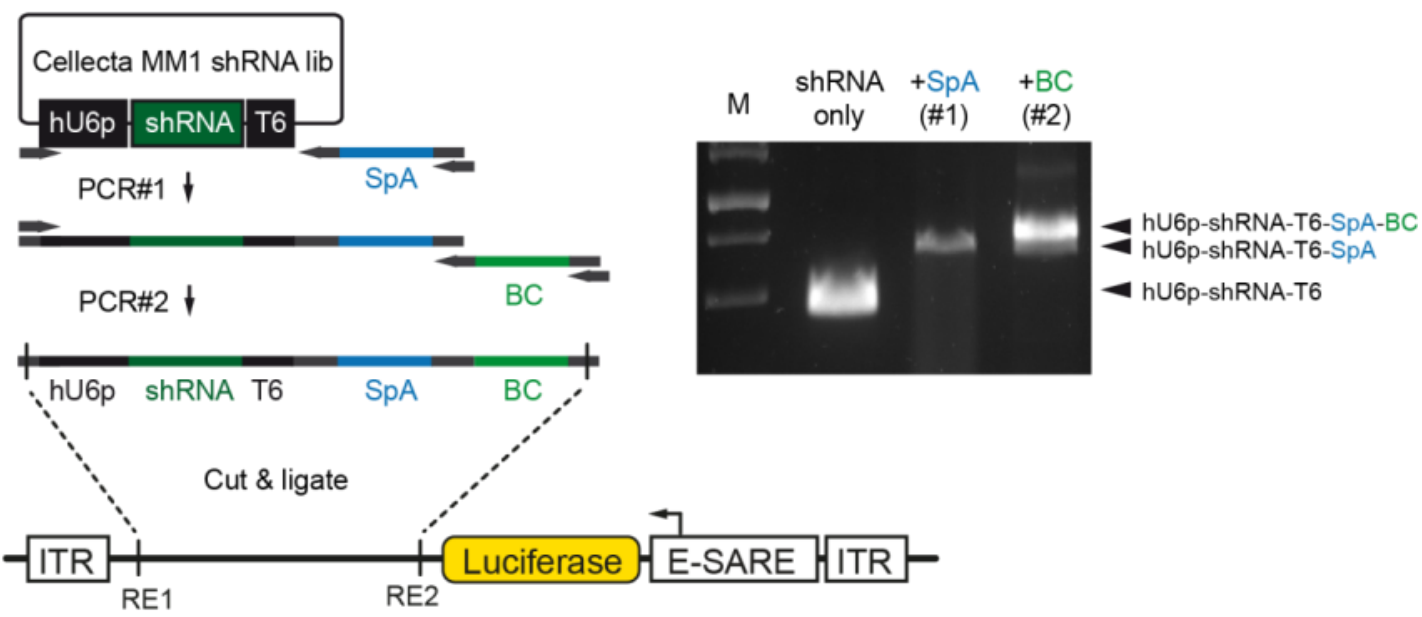

B

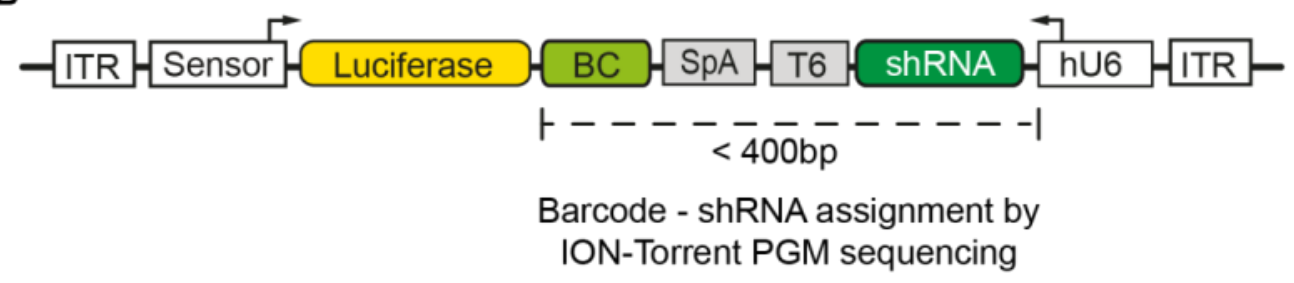

Figure 16: Generation of the PATHscreener library.

A Cloning workflow. Left, the shRNA expression cassette is amplified by PCR and extended by the SpA. A second PCR adds the barcode (BC) to the previous PCR product. The product of PCR\#2 is finally ligated into the sensor containing AAV backbone. Right, verification of the PCR products by agarose-gel electrophoresis. B Final cloning product. The proximity of barcode and shRNA allows the barcode - shRNA assignment by next-generation sequencing using the ION-Torrent PGM with 400bp chemistry. 
Table 1: Complexity of the PATHscreener library.

\begin{tabular}{ccccc}
\hline Colony no. & Barcode no. & shRNA no. & Gene no. & shRNAs/gene \# \\
\hline 30,000 & 25869 & 12780 & 4467 & 2.9 \\
\hline
\end{tabular}

\#mean value

\subsection{Sensor-based pooled RNAi screen in primary neurons}

In order to broaden the cell types and cellular processes that can be studied using pooled RNAi screens towards relevant psychiatric risk pathways in primary neurons, a new barcoded genetic sensor readout has been developed. Using an AAV library that contains the E-SARE sensor and a focused shRNA library of $~ 4500$ signaling pathway genes we aimed to perform the first pooled screen in primary neurons. The screen was designed to identify genes involved in neuronal excitation and synapse-to-nucleus signaling. The data presented in this thesis focus on two main questions. Firstly, does a pooled RNAi screen in primary neurons deliver meaningful hit lists? And secondly, is the screen reproducible?

\subsubsection{Proof-of-concept screen for regulators of neuronal excitation}

Initially two screens were conducted as a proof-of-concept. Those first two screens (A and B) were performed identically except that 10 or 5 million cells per sample were used, respectively. Each condition had 2-3 biological replicates. Cortical neurons were isolated from E15.5 wild-type mice and infected on DIV6 with the AAV pool at an AAV particle to cell ratio of 1000:1 ( $60 \%$ infection rate). On DIV10 half of the samples were treated with TTX and APV to silence the spontaneous neuronal network activity and thereby reduce the ESARE activity to baseline. The remaining samples were stimulated with a cocktail containing bicuculline, 4-AP, glycine and strychnine (BIC/4-AP) on DIV12 to boost synaptic activity (Figure 17 and Table 2). The RNA harvest time point for a maximal dynamic range was at 4 hours after BIC/4-AP application (Figure 18A). The kinetic of the RNA-based reporter is thus faster compared to the luciferase reporter which peaks at 8 hours after BIC/4-AP application (Figure 18B). All samples, silenced and stimulated, were subsequently lysed for total RNA purification. Barcode libraries were prepared for deep-sequencing on an Ion Torrent Proton sequencer. We hypothesized that the induction of a barcode, which codes for a hit shRNA, will be reduced or increased compared to the median induction of the total library. For hit nomination two analysis methods were applied: (1) Ranking by enhanced Z-scores of normalized fold changes between stimulated and silenced conditions and (2) a protocol analogous to differential gene expression analysis in RNA-seq using the DESeq2 package in R (Dai et al., 2014; Love et al., 2014). 


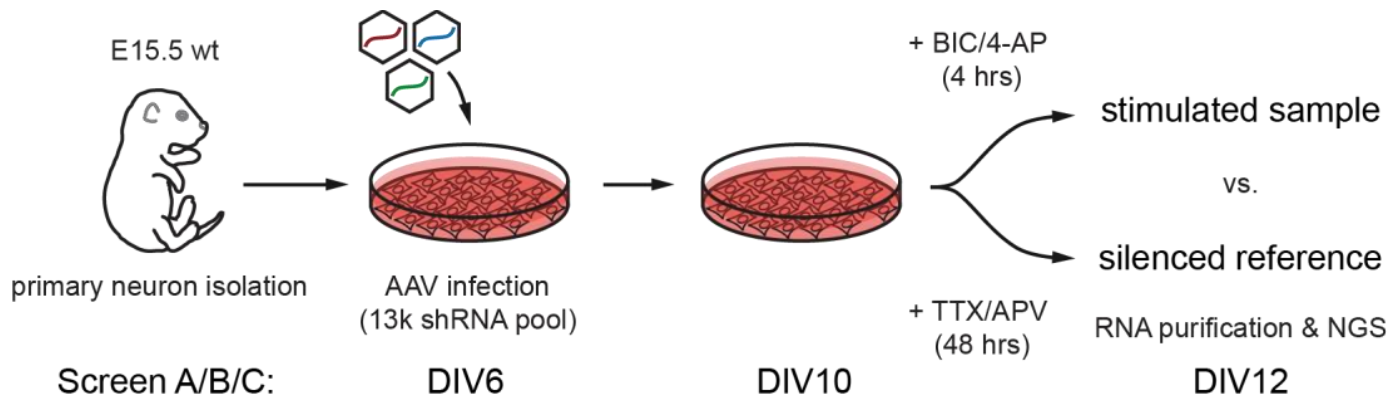

\section{Figure 17: Screening design and workflow}

Primary cortical neurons were isolated from E15.5 mice and plated in $15 \mathrm{~cm}$ (10 mio cells; Screen $A$ and $C$ ) or $10 \mathrm{~cm}$ dishes (5 mio cells; Screen B). Neurons were infected with the AAV PATHscreener library at DIV6. At DIV10 reference samples were treated with TTX/APV for the following 48 hours to reduce sensor activity. Neuronal activity is induced in the remaining cultures at DIV12 using a BIC/4-AP cocktail for 4 hours. Subsequently cultures were lysed and total RNA was purified and processed for next-generation sequencing.

Table 2: Screens in this thesis: A / B / C

\begin{tabular}{lllllll}
\hline Screen & $\begin{array}{l}\text { Cell } \\
\text { no./sample }\end{array}$ & $\begin{array}{l}\text { AAV } \\
\text { batch }\end{array}$ & Infection & Lysis & $\begin{array}{l}\text { Reference } \\
\text { condition }\end{array}$ & Test condition \\
\hline $\mathrm{A}$ & $10 \mathrm{mio}$ & $\mathrm{A} 59.1$ & DIV6 & DIV12 & TTX/APV & $\mathrm{BIC/4-AP}$ \\
$\mathrm{B}$ & $5 \mathrm{mio}$ & $\mathrm{A} 59.1$ & DIV6 & DIV12 & TTX/APV & $\mathrm{BIC/4-AP}$ \\
$\mathrm{C}$ & $10 \mathrm{mio}$ & $\mathrm{A} 59.2$ & DIV6 & DIV12 & TTX/APV & BIC/4-AP \\
\hline
\end{tabular}

\subsubsection{Quality controls within the screening pipeline}

Multiple quality control measures have been implemented into the screening workflow. Sister cultures were used to monitor the sensor activity upon neuronal silencing and stimulation with the BIC/4-AP cocktail in live cells. A $~ 21$ fold-change of the E-SARE sensor activity was measured at the peak of induction by live cell luciferase activity recordings (Figure 18B). At the cDNA level from the actual screening samples, the sensor response was controlled by qRT-PCR using barcode flanking primers (qDec primers). Relative and absolute quantification of the barcode $c D N A$ using qDec primers verified the E-SARE stimulation upon BIC/4-AP during the screen (Figure 18C and D). After total read count normalization of the raw data, pair-wise Pearson correlation and unsupervised hierarchical clustering was used to identify potential outlier samples. All biological replicate samples of screen A and $B$ correlated well and samples clustered together by condition (BIC/4-AP, TTX/APV; Figure $18 \mathrm{E})$. The highest correlations are observed between samples that were stimulated with $\mathrm{BIC} / 4-\mathrm{AP}$. 
A

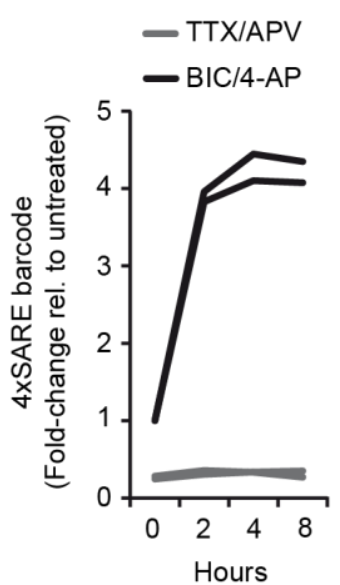

D

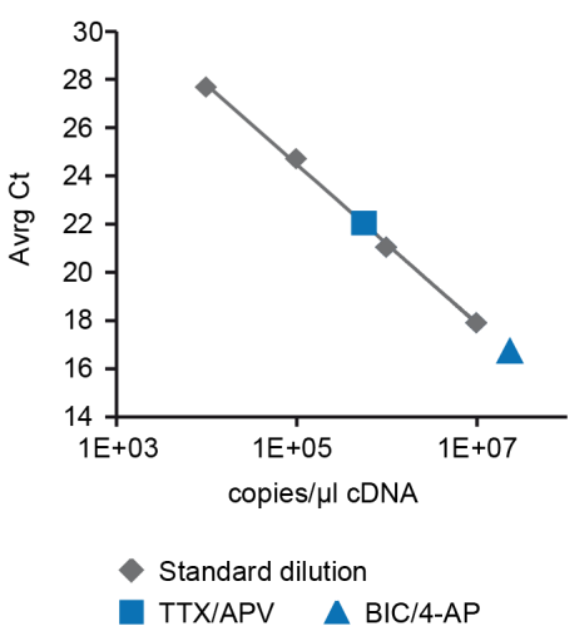

B

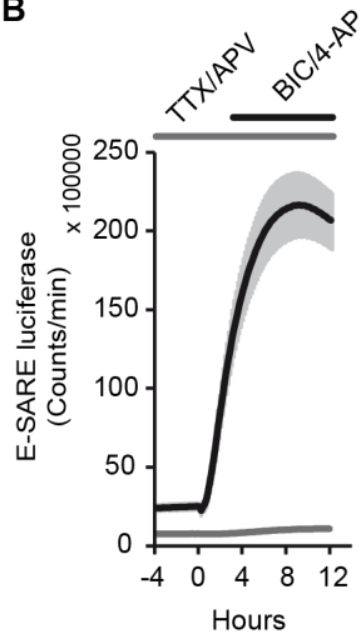

C

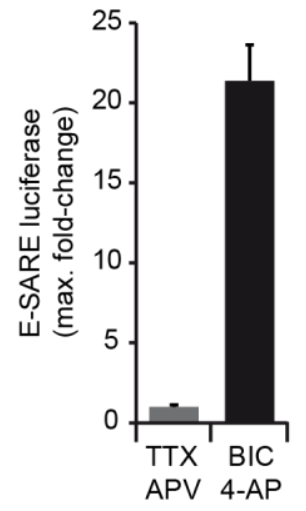

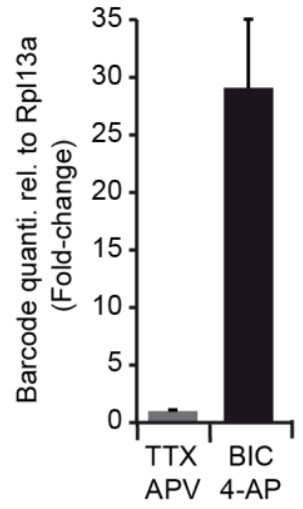

E

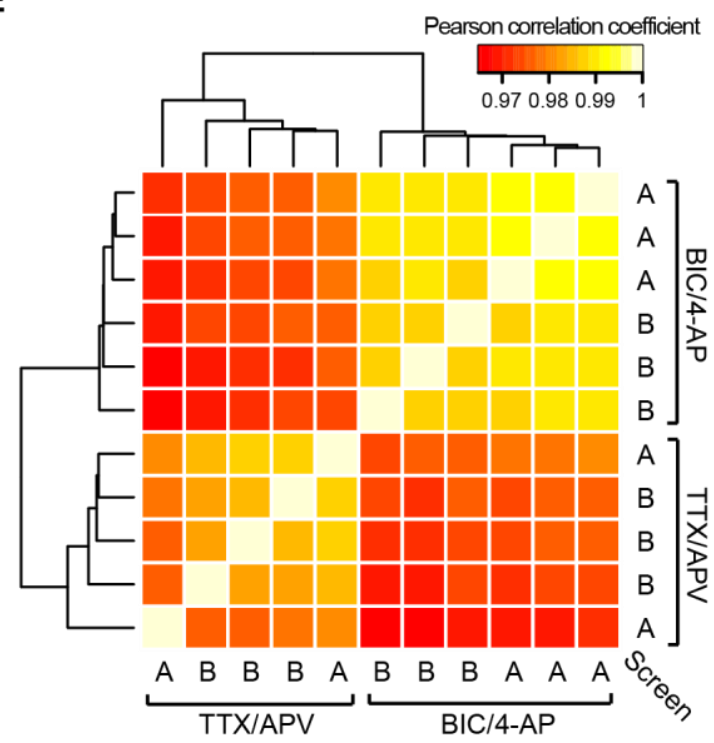

Figure 18: Quality control measures from the pooled RNAi screen.

A. Kinetics of the SARE-minMLP sensor response to TTX/APV and BIC/4-AP measured by barcode sequencing. The response for two individual barcodes is shown (average from two assay replicates; extracted from the multiplexed cis-regulatory sensor assay). B. Left, E-SARE sensor response to TTX/APV and BIC/4-AP determined by live cell luciferase activity measurement in sister cultures of screen $A / B$ ( $n=4+-$ sem). Right, maximal E-SARE luciferase induction by synaptic activity (time-point 8 hours of BIC/4-AP stimulation). C, D. Relative and absolute quantification of the barcode expression in screen $A$ in response to TTX/APV and BIC/4-AP. E. Pair-wise correlation of normalized read counts from biological replicates of screen $A$ and $B$. Clustering by unsupervised hierarchical clustering.

\subsubsection{Hit nomination by enhanced Z-score ranking and DESeq2 analysis.}

Data analysis and ranking of shRNAs and gene targets has been done by two independent methods. The knockdown effects on the E-SARE sensor activity were analyzed by an enhanced Z-score ranking and using the Bioconductor package DESeq2 (Love et al., 2014). 
Since absolute abundances of individual vectors vary within the library pool, barcode counts in the stimulated samples are always normalized to the barcode counts in the unstimulated reference samples (Figure 17). Both methods are based on the assumption that the majority of shRNAs within the library do not alter the induction of the E-SARE sensor in response to synaptic stimulation by BIC/4-AP. This assumption is valid if a shRNA library of high complexity, as in this case, is used and which was supported by the analysis of 10 random shRNA constructs (Figure 16B). The enhanced Z-score method analyzes the effect of a knockdown by reporting the deviation of the corresponding sensor activity from the median sensor activity of the entire pool. The advantage of the enhanced Z-score is its robustness against outliers which in this case are the hits. A negative enhanced Z-score of a shRNA means that the target gene is a positive regulator of the measured phenotype, whereas a positive enhanced Z-score identifies genes that act as negative regulators. Sensor activity in the screen can be shifted in general towards both directions, hence, positive and negative regulators can be identified. However, more shRNAs have a negative than a positive enhanced Z-score above the thresholds of 3/-3 (209 shRNAs at enhanced Z-score < -3; 111 shRNAs at enhanced Z-score $>3$ ) and the overall amplitude is stronger at the negative scale (Figure 19A, left). Thus, the screen appears to be more sensitive for positive regulators, which is likely due to the strong stimulation applied during the screen. In order to collapse the hit list to the gene level, the shRNA with the strongest effect was selected to represent the corresponding gene target. At this level, 151 genes have an enhanced Z-score of less than -3 (Figure 19A, right) and can be considered as primary hits for positive regulators.

A second powerful analysis strategy uses the Bioconductor R package DESeq2 (Love et al., 2014). This package was originally developed for the identification of differentially expressed genes in RNA-seq experiments. Nevertheless, it has been shown that the DESeq2 package as well as the similar edgeR package are also powerful tools for hit nomination, as genetic screening data and RNA-seq data are very similar and have a negative binominal distribution (Dai et al., 2014; Parnas et al., 2015). In this study, DESeq2 was used for read count normalization between samples and identification of differentially expressed barcodes. Significance of differential expression was tested using the Wald test and corrected by multiple-testing using the Benjamini-Hochberg method (Benjamini and Hochberg, 1995). In accordance with the results from the enhanced Z-score analysis, the DESeq2 analysis identified more shRNAs where the sensor induction is significantly downregulated (335 at a false discovery rate $(F D R)<0.05)$ compared to shRNAs with an enhanced sensor induction (250 at FDR < 0.05) (Figure 19B). Hit lists generated by both methods show a substantial overlap (Enh. Z-score vs. DESeq2 log2FC 54/100; vs. DESeq2 FDR 41/100) (Figure 19C) and the use of both strategies in parallel can increase the confidence in hit nomination. The following steps of analysis are based on the enhanced Zscore ranking unless otherwise stated. 
A
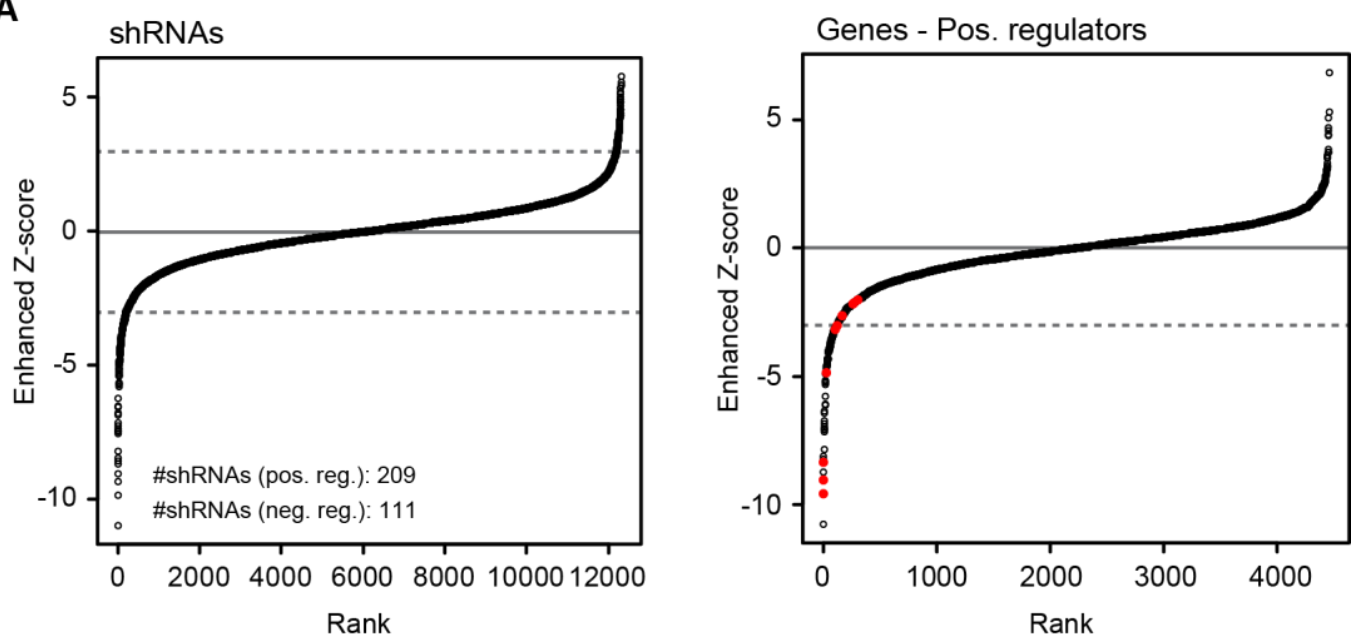

B

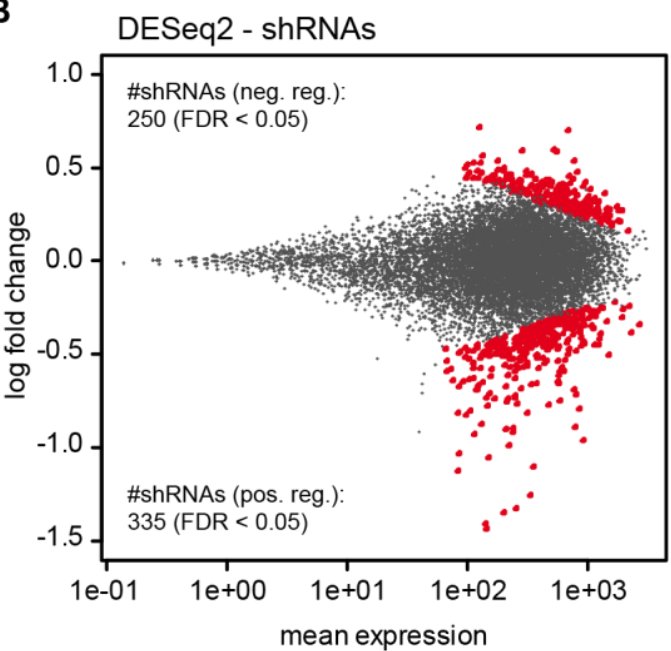

C

Top100 positive regulators Enh. Z-score vs. DESeq2

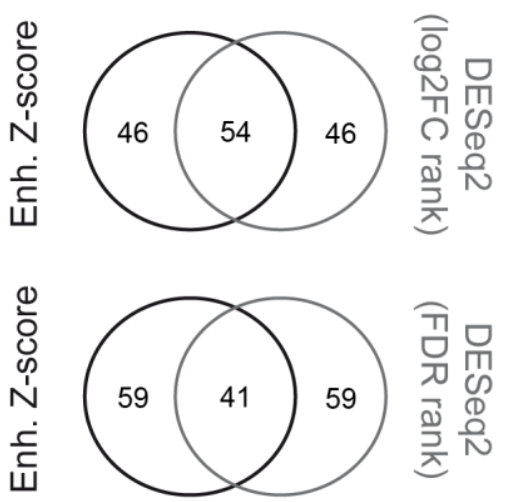

Figure 19: Enhanced Z-score and DESeq2 analysis of the pooled RNAi screen $A$.

A. Enhanced Z-score rankings. Left, For individual shRNAs from screen A. An enhanced Z-score of $+3 /-3$ is indicated by a dashed line. Right. Collapsed to gene level by filtering for the shRNA with the most negative score per gene. The dashed line indicates a score of -3 . Selected candidates for subsequent validation are highlighted in red. B. MA-plot compares for shRNAs the DESeq2 log2 fold changes (screen $A$ ) to the mean expression of the corresponding barcode. shRNAs with differentially expressed barcodes are highlighted in red $(F D R<0.05)$. C. Overlap between the top 100 positive regulators identified by enhanced Z-score analysis and by DESeq2 analysis (either ranked by log2 fold change or FDR). FDR was determined using the Benjamini-Hochberg method.

Next, 10 candidates for positive regulators were selected from screen $A$, covering an enhanced Z-score range from -2 to -10 as indicated in figure 19A (right, red dots). These candidates are components of cAMP signaling (Adcy3), cytokine signaling (II2rb), calcium signaling (Calm1, Camk2d, Cacna1f, Cacna1h, Tacr2), the circadian clock (Bhlhe40), the postsynaptic density (Gphn), and a schizophrenia risk gene (Disc1). They were selected to test the first step of validation by performing individual knockdown experiments. For each 
gene the shRNA with the strongest effect in the screen was cloned into the PATHscreener vector and tested individually for its interference in BIC-induced synaptic signaling (Figure 20). All tested shRNAs reduced the E-SARE induction compared to a non-targeting control (NTC) vector and 8 out of 10 shRNAs showed a significant effect (student's t-test, $p<0.05$ ). While this is the first validation step, additional orthogonal validation tools (e.g. synapse/dendrite stainings, electrophysiology) need to be implemented into the protocol and high-content analysis of hit candidates might be a powerful strategy.

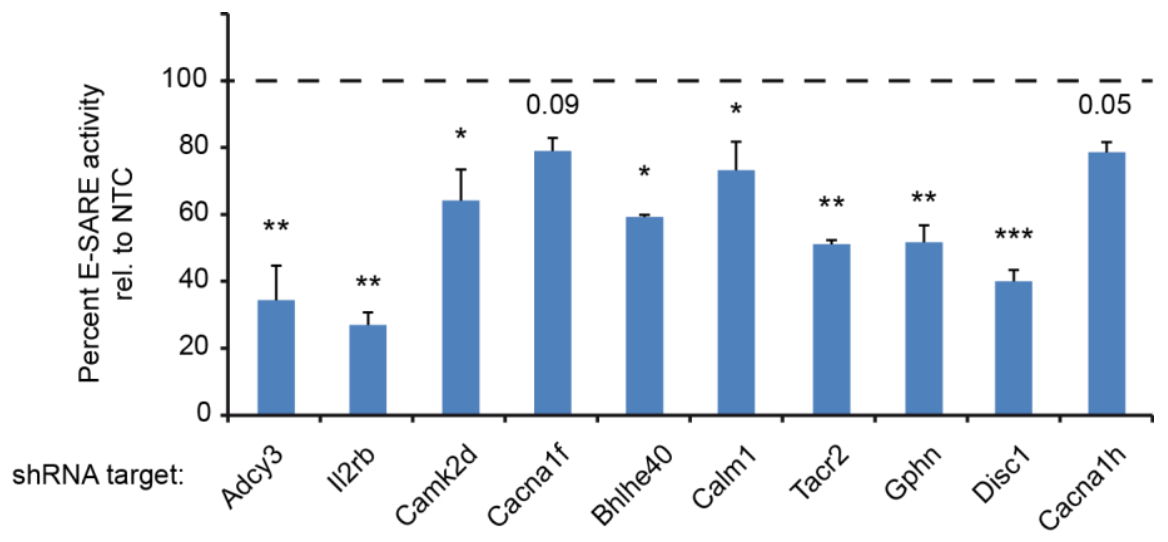

Figure 20: Individual validation of selected candidates.

E-SARE activity in response to knockdown of 10 candidate genes relative to a non-targeting control (NTC). Primary cortical neurons were infected with individual PATHscreener vectors for 10 different shRNAs selected from screen A and a NTC vector. In accordance to the screening conditions cultures were either silenced by TTX/APV ( $48 \mathrm{hrs}$ ) or stimulated by BIC/4-AP ( $4 \mathrm{hrs}$ ) and E-SARE activity was determined by luciferase measurement $(n=3+-s d)$.

\subsubsection{Hit ranking to biological function by KEGG and Reactome analyses}

The main focus of this proof-of-concept study lies on the quality assessment of the screening results by comparison to currently available data. One would assume that in particular a knockdown of synaptic genes and genes with known function in cAMP/calcium signaling are likely to affect synapse-to-nucleus signaling and therefore alter E-SARE induction. In order to systematically interpret and validate the output of the pooled RNAi screen for regulators of neuronal excitation and synapse-to-nucleus signaling, the two manually curated pathway databases KEGG and Reactome were used as a reference (Fabregat et al., 2016; Ogata et al., 1998). This analysis has been done with the top 140 positive regulators identified in screen A. Strikingly, pathway analysis using the KEGG database revealed an enrichment of genes associated with the categories "Long-term potentiation", "Neurotrophin signaling", and "Calcium signaling" ( $p<0.05$, hypergeometric test, Table 3 ). These associations confirm that 
the screen produces reliable results and can be used to identify pathway genes in primary neurons.

Table 3: KEGG Pathway analysis for the Top140 positive regulators

\begin{tabular}{|c|c|c|c|}
\hline KEGG Pathway & $\mathrm{R}$ & pValue\# & Genes \\
\hline $\begin{array}{l}\text { Long-term } \\
\text { potentiation }\end{array}$ & 4.15 & 0.0013 & $\begin{array}{l}\text { Calm1, Ppp1ca, Adcy8, Araf, Camk2d, } \\
\text { Ppp1cb, Camk4 }\end{array}$ \\
\hline $\begin{array}{l}\text { Neurotrophin } \\
\text { signaling }\end{array}$ & 2.94 & 0.0032 & $\begin{array}{l}\text { Calm1, Psen1, Camk2d, Nfkbie, Pik3cd, } \\
\text { Mapk14, Csk, Camk4, Fasl }\end{array}$ \\
\hline Calcium signaling & 2.08 & 0.0374 & $\begin{array}{l}\text { Calm1, Cacna1f, Slc25a4, Tacr2, Camk2d, } \\
\text { Adyc8, Camk4, Adcy3 }\end{array}$ \\
\hline Chemokine signaling & 2.05 & 0.0406 & $\begin{array}{l}\text { Pik3cd, Ccr5, Adyc8, Cxcr5, Xcr1, Csk, Cxcl2, } \\
\text { Adcy3 }\end{array}$ \\
\hline MAPK signaling & 1.79 & 0.0519 & $\begin{array}{l}\text { Dusp6, Cacna1f, Ppp5c, Fgf20, Fgf12, } \\
\text { Mapk14, Fgf18, Daxx, Map3k12, FasI }\end{array}$ \\
\hline
\end{tabular}

R Enrichment ratio; \# hypergeometric test

Along with pathways of known and well-established function in neuronal activity-dependent signaling, surprisingly, an enrichment for genes in the KEGG pathway 'Chemokine signaling” was found (Table 3). The top hit list for positive regulators contains the chemokine receptors CC-motif-chemokine receptor 5 (Ccr5), CXC-motif-chemokine receptor 5 (Cxcr5), and chemokine ( $\mathrm{C}$ motif) receptor $1\left(X_{C r} 1\right)$, as well as, the ligand chemokine (C-X-C motif) ligand 2 ( $\mathrm{Cxc} / 2$ ). In addition, the C-Src kinase (Csk), two adenylate cyclase isoforms 3 and 8 (Adcy3/8), and the catalytic domain delta of PI3-kinase (Pik3cd) have been identified as components of chemokine signaling.

A second line of evidence for the functionality of the screen comes from a pathway analysis using the Reactome pathway browser. Significant enrichments were found for multiple signaling events that involve the second messenger molecules calcium, diacylglycerol (DAG), and inositol triphosphate (IP3). In addition, the Reactome analysis reflects that in primary cortical cultures primarily the glutamatergic post-synapse is screened (Pathway: "Activation of NMDA receptor upon glutamate binding and postsynaptic events", $p=0.003$ ). Finally, the Reactome pathway analysis revealed that the screen is also sensitive for perturbed neurodevelopmental processes like neurite outgrowth (Pathway: "NCAM signaling for neurite outgrowth", $p=0.004$ ) (Table 4). 
Table 4: Reactome Pathway analysis for the Top140 positive regulators

\begin{tabular}{lll}
\hline Pathway & pValue & FDR \\
\hline Phospholipase C-mediated cascade; FGFR3/4/2/1 & $6.69 \mathrm{E}-05 / 7.54 \mathrm{E}-05 /$ & 0.022 \\
Activation of CaMK IV & $1.46 \mathrm{E}-04$ / 9.14E-04 & 0.022 \\
PLC beta mediated events & $4.36 \mathrm{E}-04$ & 0.022 \\
G-protein mediated events & $5.66 \mathrm{E}-04$ & 0.022 \\
CaM pathway & $6.26 \mathrm{E}-04$ & 0.022 \\
Calmodulin induced events & $6.76 \mathrm{E}-04$ & 0.022 \\
Ca-dependent events & $6.76 \mathrm{E}-04$ & 0.022 \\
CaMK IV-mediated phosphorylation of CREB & $8.89 \mathrm{E}-04$ & 0.022 \\
DAG and IP3 signaling & $9.71 \mathrm{E}-04$ & 0.026 \\
CREB phosphorylation through the activation of & 0.001 & 0.031 \\
CaMKK & 0.002 & 0.031 \\
PLC-gamma1 signaling & 0.002 & 0.034 \\
Post NMDA receptor activation events & 0.002 & 0.050 \\
Activation of NMDA receptor upon glutamate binding & 0.003 & 0.062 \\
and postsynaptic events & 0.004 & 0.076 \\
NCAM signaling for neurite outgrowth & 0.005 & 0.076 \\
\hline MAPK family signaling cascade & 0.005 & \\
CREB phosphorylation through the activation of & & \\
adenylate cyclase &
\end{tabular}

Figure 21 summarizes the major findings from screen $A$ by mapping hit candidates along the synapse-to-nucleus route. Mapping was done using the KEGG pathway maps "Long-term potentiation", "Glutamatergic synapse", "Calcium signaling pathway", "mTOR signaling pathway", and "PI3K-Akt signaling pathway". The map has been further complemented by a few entries based on additional literature. As expected from the KEGG and Reactome pathway analysis output many positive regulators that ranked high in the screen are involved in post-synaptic function. Along the route from the synapse to the nucleus multiple genes involved in calcium signaling, a schizophrenia risk pathway, were found (Cacna1f/h/g/c, Calm1/3, Camk2d/g, Camk4) and with Itpr1 an IP3 receptor involved in calcium wave propagation along the dendritic ER. Besides calcium regulators, several members of the mTOR and PI3K-Akt signaling pathways, which are associated to schizophrenia and ASD as well, ranked high in the screen (e.g. Pik3cd, Akt1, mTOR) (Kim et al., 2009; Sawicka and Zukin, 2012). Notably, the recently identified schizophrenia risk gene $C 4 a$, which is member of the classical component cascade, ranked high in the screen (rank 446, screen A; rank 50, screen B) (Sekar et al., 2016). 


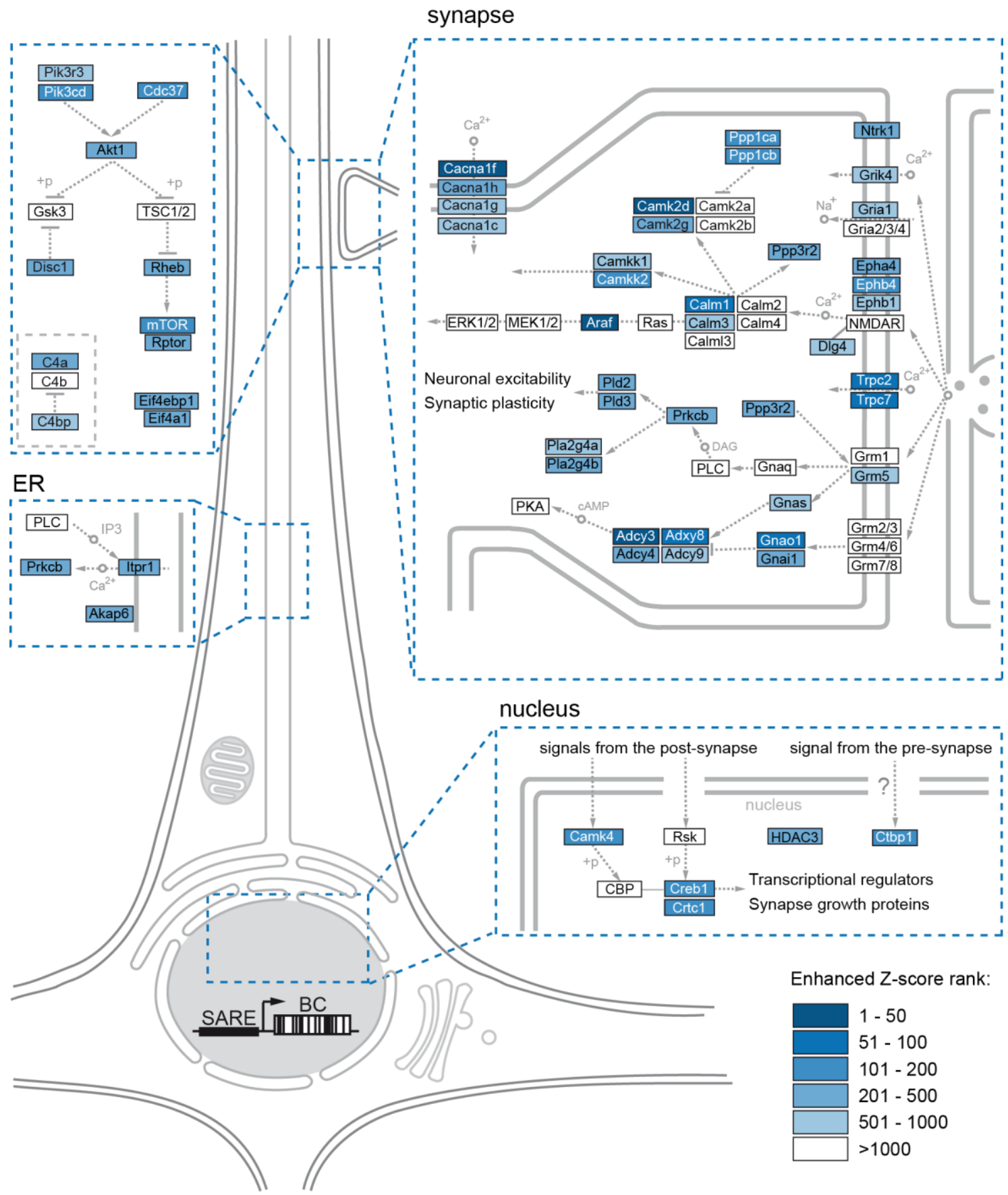

Figure 21: Screening hits along the synapse-to-nucleus pathway.

Pathway mapping of positive regulators of neuronal excitability and synapse-to-nucleus signaling based on KEGG and manual literature mining. The color code indicates the enhanced Z-score ranking in screen $A$.

\subsubsection{Reproducibility of the screen}

The robustness of a screen and consequently the reproducibility of its results should always be a major concern during assay development. Nevertheless, the issue of insufficient 
reproducibility in biomedical research is frequently raised in academia and industry (Freedman et al., 2015). The presented pooled sensor-based RNAi screen in primary neurons was performed three times in total in order to estimate the degree of reproducible results. Screen $A$ and $B$ were performed in parallel with the same batch of primary neurons and AAV preparation. Both screen only differed in terms of cell number (screen A: 10 mio cells/sample; screen B: 5 mio cells/sample). Screen C has been carried out completely independent. This means that primary neuron cultures were prepared on another day using different embryos, a new AAV batch was used, and multiple steps of the screening protocol were performed by another experimenter (e.g. primary neuron preparation, RNA isolation, cDNA synthesis). Each screen has been completed with 2-3 internal biological replicates per condition. Comparison of the enhanced Z-scores from the entire gene level results from screen $A$ and $B$ displayed that both datasets correlate with a spearman-rank coefficient (rho) of 0.567 (Figure 22A). A focused view at the top hits for positive regulators revealed a substantial overlap between screen $A, B$, and $C$. The 100 highest-ranked positive regulators from screen $A$ and $B$ share over $50 \%$ of genes when analyzed by DESeq2 and ranked by log2 fold-change (log2FC) or FDR (Figure 22B). Analysis by enhanced Z-score ranking displayed an overlap of 45 genes between screen $A$ and $B$ and 38 genes between screen $A$ and $\mathrm{C}$ out of the top 100 (Figure 22C).

The evaluation of all three screens indicates that in particular the discovery of positive regulators of neuronal excitation under strong synaptic stimulation is reproducible.

A

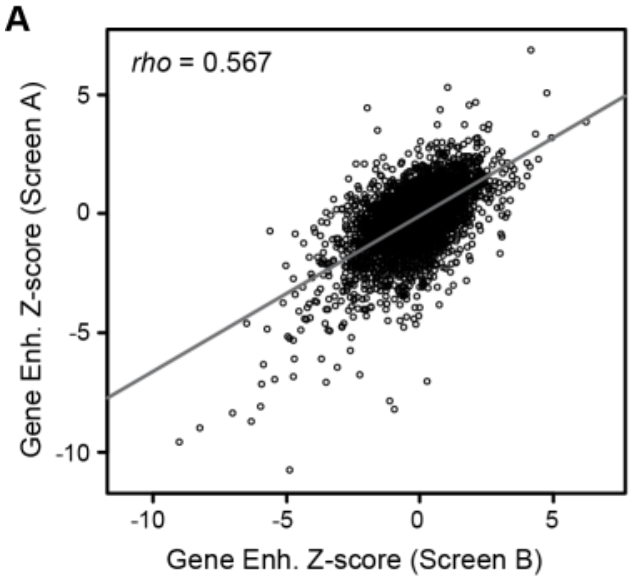

B

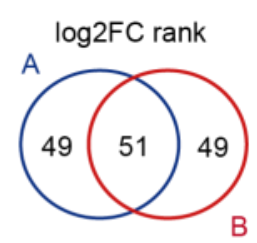

DESeq2: Top100 positive regulators

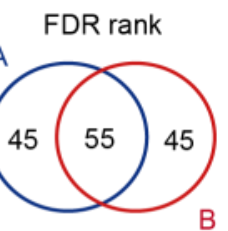

C

Enh. Z-score: Top 100 positive regulators

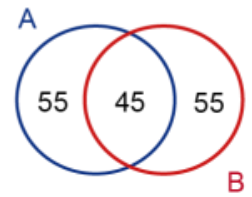

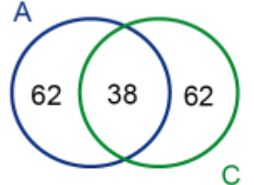

Figure 22: Reproducibility of the pooled RNAi screen in primary neurons.

A. Scatterplot of enhanced Z-scores from screen A and B. Data has been collapsed to gene level and filtered for positive regulators. The spearman-rank coefficient rho and linear regression are indicated. B, C. Venn diagrams for the overlap in the hit lists generated by DESeq2 analysis (B) and enhanced Z-score ranking (C). 


\subsubsection{Screening for negative regulators of neuronal excitation}

The identification of negative regulators of neuronal excitation and synapse-to-nucleus signaling is of great interest particularly for the discovery of new potential drug targets in order to restore hypoexcitability and synaptic function of neurons. As pointed out earlier, hit nomination for negative regulators is challenging due to a smaller dynamic range of the $\mathrm{E}$ SARE sensor under strong synaptic stimulation, hence, false-positives are more likely. Consequently, datasets from screen $\mathrm{A}, \mathrm{B}$, and $\mathrm{C}$ were used and compared in order to evaluate the screening performance for negative regulators. As expected, the pairwise overlap as well as the overlap between all three screens is smaller than for positive regulators. Within the top 100 potential negative regulators, screen $A$ and $B$ shared 15 genes, screen $A$ and $C$ shared 11 genes, and screen $B$ and $C$ shared 10 genes (Figure 23). One gene is in common to the hit lists of all three screens. Among hits found in two independent screens are genes with known function in neuronal excitation and calcium/cAMP-signaling like the $\mathrm{Na}^{+} / \mathrm{H}^{+}$antiporter S/c9a1, the ATPase $\mathrm{Na}+/ \mathrm{K}+$ transporting subunit beta 3 (Atp1b3), and the cAMP-responsive element modulator (Crem) (Forrest, 2014; Gu et al., 2001; Mellström et al., 1993; Xia et al., 2003). The only gene that is shared across all three hit lists for negative regulators is Katnb1 which encodes the noncatalytic regulatory p80 subunit of katanin, a protein complex involved in severing of microtubules (Hartman et al., 1998). Hence, Katnb1 knockdown might stabilize neurites, thereby enhancing the excitatory input.

Top100 Negative regulators

(Enh. Z-score)

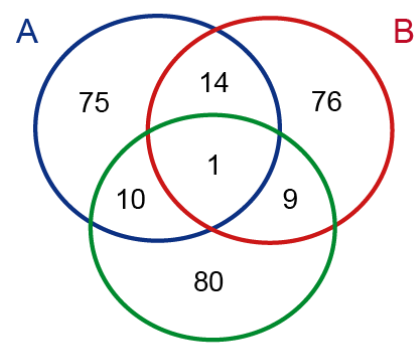

C

Figure 23: Comparison of identified negative regulators in screen A, B, and C.

Venn diagram showing the overlap between the top 100 potential negative regulators from screen $A, B$, and $C$ identified by enhanced $Z$-score ranking. 


\subsection{Adaptation towards CRISPR-Cas9-based screening in primary neurons}

Over the last three years CRISPR-Cas9 has evolved to the most popular and powerful tool not only for genome engineering but also for loss- and gain-of-function studies (Gilbert et al., 2014; Jinek et al., 2013; Larson et al., 2013). Similar to the early days of pooled RNAi screens, it is no surprise that the cancer field is again at the forefront of functional genomics screens using CRISPR-Cas9. To date, multiple pooled CRISPR-Cas9 screens have been published. Most of them have been performed in cancer cell lines but recently one screen in primary dendritic cells using a pathway readout has been published (Parnas et al., 2015; Shalem et al., 2014, 2015; Wang et al., 2014). Direct and indirect comparisons between pooled RNAi and CRISPR-Cas9 screens have indicated that CRISPR-Cas9 has superior ontarget efficacy and reduced off-target activity (Evers et al., 2016a; Morgens et al., 2016; Shalem et al., 2014). Although most of the published screens are loss-of-function knockout screens, a major advancement is its use for gain-of-function studies. This for the first time allows genome-wide gain-of-function screens in mammalian cells and hence complements the loss-of-function toolbox (Konermann et al., 2015).

Based on the acquired expertise from the proof-of-concept pooled RNAi screen in primary neurons, the adaptation towards a CRISPR-Cas9-based screening tool has been initiated. Transcriptional regulation by CRISPR-Cas9 relies on the recruitment of effector domains (e.g. p65, VP64 activator domains) into proximity of the transcriptional start site of the geneof-interest. It has been shown that recruitment of multiple domains to the same locus enhances activation of gene expression (Konermann et al., 2015; Tanenbaum et al., 2014). We therefore decided to use the CRISPR-Cas9-SAM (Synergistic Activation Mediator), that recruits multiple effector domains using a fusion of the sgRNA with two MS2 aptamer sequences and a second fusion of the MS2-binding domain with the effector domains (e.g. p65 and VP64) (Figure 24A). This increases the number of effector domains at the locus compared to a direct fusion of the effector domain to dCas9 (as illustrated in Figure 6B and C). Two AAV vectors have been cloned, one for dCas9 (Streptococcus pyogenes) expression and the second vector represents the CRISPRa PATHscreener2.0 vector with the following modifications to the RNAi-based PATHscreener vector: (1) The firefly luciferase was substituted by the shorter NanoLuc luciferase. (2) The shRNA is substituted by the sgRNA2.0. (3) A third expression cassette is introduced for MS2-p65-VP64 expression (Figure 24B, PATHscreener2.0). Multiple experiments have been performed until now to validate the CRISPRa PATHscreener2.0 vector. Expression of dCas9 and MS2-p65-VP64 from the AAV backbone has been verified by western blot (Figure 24C). Stimulation of the ESARE sensor within the PATHscreener2.0 backbone by PMA in HEK293 cells showed comparable inductions as with the RNAi-based PATHscreener vector (Figures 15A and 24D). The system is able to induce expression of a luciferase reporter as well as 
endogenous Arc mRNA (Figure 24E and F). Primary neurons express both dCas9 and MS2p65-VP64 after AAV infection. This has been a critical step as the AAV-dCas9 genome slightly exceeds the optimal AAV packaging capacity of $4.8 \mathrm{~kb}$. Importantly both proteins are localized in the nucleus (Figure 24G).
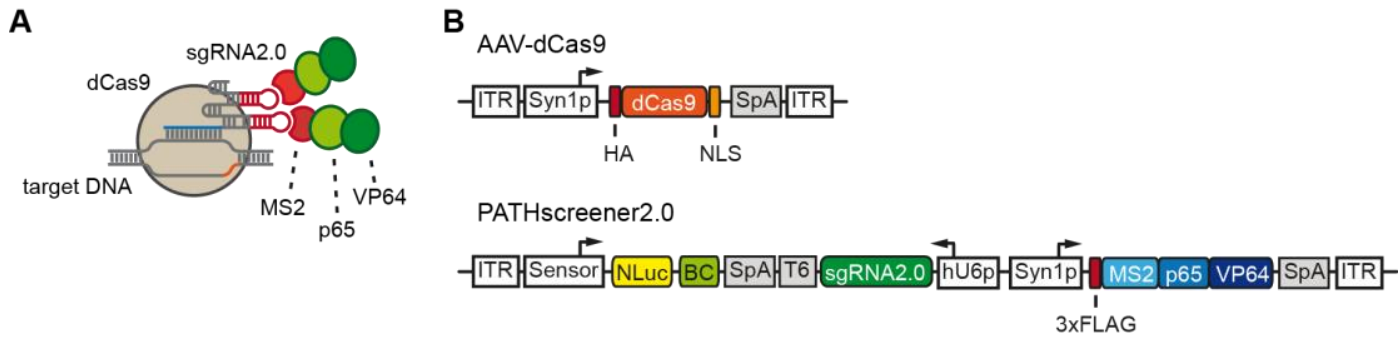

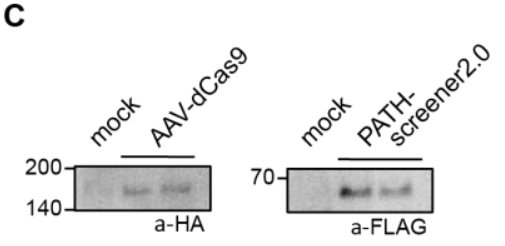

E

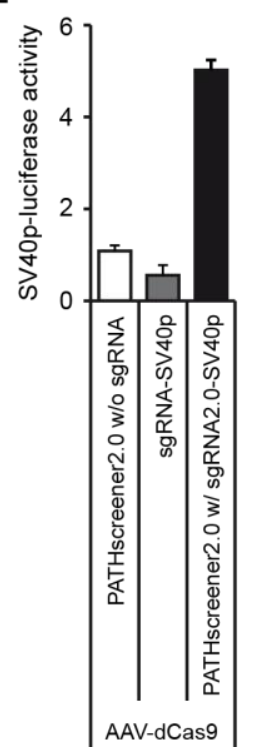

$\mathbf{F}$

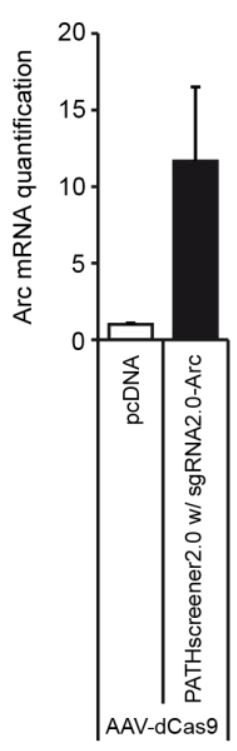

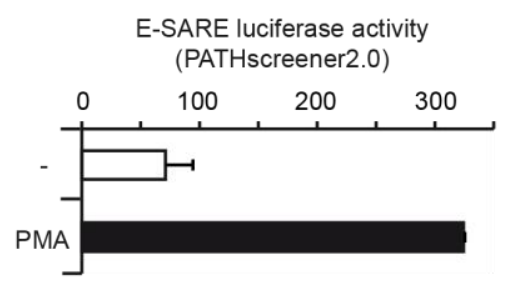

G

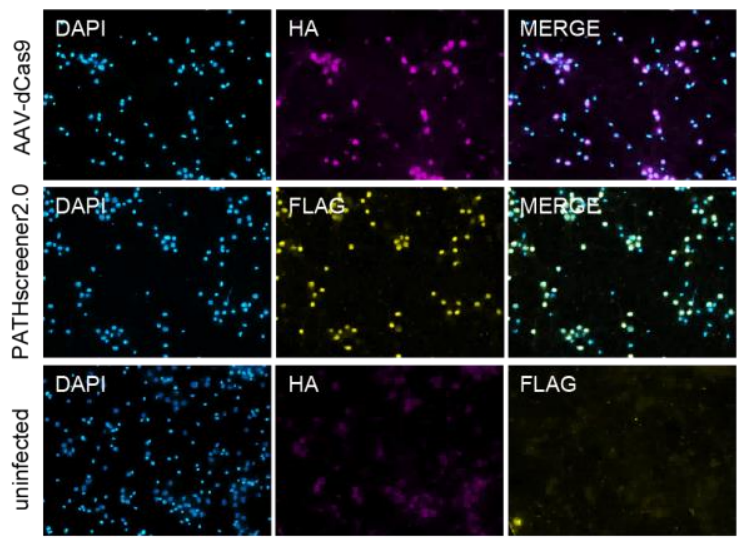

Figure 24: Adaptation towards an AAV CRISPR screening vector.

A Schematic of the CRISPRa complex comprising the target DNA bound by the inactive Cas9 (dCas9) and the sgRNA2.0 harboring two MS2 loops. MS2 loops are recruiting the MS2-binding domain fused to the p65 and VP64 transcription activator domains. B AAV maps of the dCas9 vector (top) and the CRISPRa-PATHscreener vector (bottom). C Expression validation of dCas9 and MS2-p65-VP64 in HEK293FT cells by western blot. D Validation of the E-SARE sensor response to PMA in the context of the CRISPRa-PATHscreener vector in HEK293 cells $(n=6+$ +sd). E Transcriptional activation of a SV40p-luciferase reporter by dCas9, MS2-p65-VP64 and sgRNA2.0-SV40 ( $\mathrm{n}=6+$ +- sd). F Transcriptional activation of Arc gene expression in N2a cells by dCas9, MS2-p65-VP64 and sgRNA2.0-Arc ( $n=3+-\mathrm{sd})$. G Validation of nuclear dCas9 and MS2p65-VP64 localization in primary mouse neurons after AAV infection of the vectors shown in $B$. 


\section{Discussion}

Experience-driven neuronal activity is shaping the brain throughout life by modulating synaptic connections and the excitability of neurons within circuits. Aberrant neuronal connectivity and activity might result from a multitude of genetic defects and is likely causative for various CNS diseases including psychiatric disorders (Grant, 2012). The underlying molecular mechanisms of neuronal activity and synaptic plasticity in health and disease can be studied using primary neuronal cultures. While research over the last decades has identified many key components of synaptic transmission, plasticity, and signaling to the nucleus, it is likely that proper neuronal function is also dependent on various other biological processes. One example is the energy metabolism and control of redox state (Sada et al., 2015; Winkler and Hirrlinger, 2015). Elucidation of the networks which affect neuronal function can be a tedious endeavor. Pooled genetic loss-of-function screens are a tool that offers a rapid and holistic view at genes with essential functions during the development of a particular cellular phenotype. Until now, no strategy for a pooled genetic screen in primary neurons has been published. The presented work describes the first proofof-concept pooled RNAi screen in primary neurons, which is also the first screen using a post-mitotic cell type. The intention for this project was dual-purpose. Firstly, the assay should be useful for the dissection of signaling pathways. Most of the time, follow-up investigations of a screen only pursue a single candidate. This constrains the full capacity of a large-scale screen. An exception is the work by Parnas and colleagues who aimed to dissect the LPS/TIr4-to-Tnf pathway in dendritic cells using a pooled CRISPR-Cas9 knockout screen (Parnas et al., 2015). This screen will be discussed and compared to the presented screen in more detail later. Secondly, an assay was to be delivered that complement the toolbox for psychiatric and neurodegenerative drug discovery in the future.

\subsection{Advancing the current screening strategies}

The current strategies for pooled genetic screens exclusively rely on a lentiviral-based approach where DNA barcodes or the shRNAs/sgRNAs itself are used for deconvolution of the cell pool by next-generation sequencing. This rather static reporter makes it indispensable to physically segregate cell populations according to their phenotype. For post-mitotic cell types or phenotypes other than proliferation it is therefore required to stain and sort cells by flow cytometry. In this work a new readout strategy for pooled genetic screens is presented which makes cell sorting unnecessary. Molecular RNA barcodes which are expressed by a pathway sensor and code for a specific shRNA allow direct deconvolution of the complete cell pool. The genetic E-SARE sensor has been empirically selected as a superior sensor for neuronal activity from a resource of 70 sensors, which is in 
line with data obtained by others (Kawashima et al., 2009, 2013). This pool of genetic sensors together with additional putative sensors described in the literature represents a resource for the dissection of numerous cellular phenotypes in the future using a pooled screen. Interesting pathways/phenotypes in the context of psychiatric and neurodegenerative diseases would be antioxidant response (ARE sensor), hypoxia (HRE sensor), hippo signaling (TEAD sensor), Wnt signaling (TCF-LEF sensor), unfolded protein response (ERSE sensor), and apoptosis (TP53INP1 sensor).

An inherent risk of high-throughput screens is the false-positive hit nomination due to interference with the assay system (e.g. reporter expression) itself. For genetic screens using reporters (e.g. GFP), this risk encompasses interference with transcription, mRNA transport and stability, translation, protein folding and degradation of the reporter protein. The integration of an RNA reporter system not only generates faster kinetics but also eliminates several of the above mentioned interference points associated to the expression of a protein reporter.

\subsection{Assay validity}

The main focus in data analysis of this proof-of-concept screen was to assess the validity of the assay. This has been done by mapping the hit list to the KEGG and Reactome pathway databases and a literature search for associations between screen hits and psychiatric disorders.

\subsubsection{Identification of genes with known function in synaptic plasticity}

Pathway analysis using KEGG and Reactome databases revealed that the screening hits are enriched for genes involved in regulation of synaptic plasticity and signaling from the glutamatergic postsynapse to the nucleus. It was expected that in particular regulators of calcium and CAMP signaling could be identified to provide evidence for the validity of the screen. This was indeed the case as multiple voltage-gated calcium channel subunits, calmodulin isoforms, adenylate cyclases, calcium/calmodulin-dependent kinases and downstream transcription factors were amongst the top-ranked genes. This confirms that the approach is suitable to dissect neuronal signaling pathways. Nevertheless, the screening results did not entirely recapitulate the pathway maps found for example in the KEGG database. This might have several reasons. First of all a focused shRNA library was used which does not cover the entire mouse genome. Second, if a certain regulator is covered but does not score in the screen, the knockdown might not be effective enough to produce a measureable phenotype. A third reason, which might contribute to false negatives, is 
functional redundancy of proteins, a general challenge to loss-of-function studies. While the biological space of the screen can be increased relatively easily up to a genomic scale by expanding the library, studying protein redundancy and epistasis would require applying a second genetic perturbation on top of the shRNA library. Although, this has even been done in a systematic manner by generating a double-shRNA library, it rapidly increases the library complexity to a point where screenings in primary neuron cultures are not feasible anymore (Bassik et al., 2013)

\subsubsection{Hits with association to psychiatric disorders}

The identification of genetic risk loci for psychiatric disorders like schizophrenia, BPD and ASD by genome-wide association studies and exome sequencing has highlighted the synapse proteome as well as several regulatory networks (e.g. calcium pathway, $\mathrm{PI3K} / \mathrm{Akt} / \mathrm{mTOR}$ pathway) as being important for the pathophysiology (Cross-Disorder Group of the Psychiatric Genomics Consortium et al., 2013; Fromer et al., 2014; Geschwind and Flint, 2015; Schizophrenia Working Group of the Psychiatric Genomics Consortium, 2014). Common variants associated with psychiatric disorders are often found in regulatory elements (e.g. enhancers), hence, altering the expression level of a gene (Maurano et al., 2012). Having the list of risk loci, it is about time to identify the genes underlying the risk association at a given locus. In patients it is the sum of numerous genetic variations which in the end might perturb brain function leading to the manifestation of psychiatric symptoms. I therefore wondered whether the knockdown of a single putative risk gene per neuron during the screen can be sufficient to generate a measureable phenotype using the E-SARE sensor. Strikingly, multiple genes are amongst the high ranking candidates which have a risk association to psychiatric disorders. Adenylate cyclase 3 (Adcy3), the top ranked positive regulator hit in screen $A$ and $B$ (enhanced Z-score rank: 2 and 1, respectively) is suggested to have a genetic association with major depressive disorder (MDD) (Wray et al., 2012). Although Adcy3 does not yet reach genome-wide significance in GWAS, it is likely to become a significant association with increasing case-control numbers. Several genes linked to schizophrenia, BPD, and/or ASD reduced synaptically-evoked E-SARE induction. These include for example Disc1, members of the PI3K/Akt/mTOR pathway (Akt1, mTOR), components of the metabotropic glutamate receptor 5 complex (Grm5, Aldoa), the dualspecificity tyrosine-(Y)-phosphorylation-regulated kinase $1 \mathrm{~A}(D y r k 1 a)$, and the actin binding LIM protein 1 (Ablim1) (Emamian et al., 2004; 2004; Kim et al., 2009; Kirov et al., 2012; Newell and Matosin, 2014; Nurnberger JI et al., 2014; Sawicka and Zukin, 2012). Notably, knockdown of multiple genes of the complement system (C4a, C4bp, C1s) ranked high as positive regulators. The notion that the classical complement cascade has a role in synapse biology is relatively new (Stephan et al., 2012). For a long time schizophrenia's association 
to the MHC locus could not be explained and the identification of the underlying risk gene was a challenge. Only very recently, C4a was identified as the main risk gene at the extended MHC locus (Sekar et al., 2016). The pooled RNAi screen supports this finding and the consent between both studies argues that pooled genetic screens might facilitate the identification of risk genes at GWAS loci. With presenilin 1 (Psen1), a prominent risk gene for Alzheimer's disease (AD) can be found in the hit lists for positive regulators (Sherrington et al., 1995). Psen 1 encodes one out of four core proteins that form the $y$-secretase complex. The $y$-secretase complex is involved in the generation of amyloid-beta peptide from amyloid precursor protein (APP), which is believed to be crucial in the pathophysiology of AD (De Strooper, 2007). Other $y$-secretase substrates are notch and neuroligin 1 (NRG1). Notch signaling has a known function in neurite outgrowth and the NRG1-ERBB4 pathway is associated to schizophrenia (Berezovska et al., 1999; Stefánsson et al., 2003). How exactly Psen1 loss-of-function mutations finally contribute to $A D$, and which substrates are critical, remains uncertain.

\subsubsection{Chemokine signaling: Growing evidence for its role in neuronal activity}

Chemokine signaling is well known for its versatile function in the haematopoietic/lymphopoietic system in general, and in particular for its role in attracting leukocytes to sites of tissue lesions (Ma et al., 1999; Rossi and Zlotnik, 2000; Szekanecz and Koch, 2001). Approximately 50 chemokines and 20 chemokine receptors are described to date and the receptors are exclusively GPCRs (Bachelerie et al., 2014). Chemokine receptors can either be stimulated by a single, specific ligand (e.g. XCR1 and its ligand XCL1), or by multiple different chemokines (e.g. CXCR2 with six ligands, including CXCL2) (Tran and Miller, 2003). Downstream signaling of chemokine receptors can activate various intracellular substrates, such as adenylate cyclases, phospholipases, GTPases as well as the MAPK and PI3-kinase pathways (Balkwill, 1998; Mellado et al., 2001; Neves et al., 2002). Hence, it is conceivable that chemokine-induced activation of these signaling pathways might play a regulatory role in neuronal plasticity, for instance. In the CNS, chemokine signaling has been mainly described in the context of neuroinflammation, which involves the recruitment of chemokine receptor-expressing microglia by chemokine-releasing neurons (de Haas et al., 2007; Ubogu et al., 2006). However, a body of evidence, from studies using various neuronal cell types (e.g. hippocampal, Purkinje, and dopaminergic neurons), suggests that chemokine signaling might modulate neurotransmission and synaptic plasticity (Bertollini et al., 2006; Giovannelli et al., 1998; Guyon et al., 2006; Ragozzino et al., 2002). In this case, chemokine signaling might occur between neurons or as autocrine signaling, which would be a possible scenario within a primary neuron culture during the pooled RNAi screen (de Haas et al., 2007). Amongst other components of 
chemokine signaling, the screen has discovered XCR1, a chemokine receptor without a known link to neurons, as a positive regulator of neuronal activity. This finding is supported by replication in screen $A$ and $B$, using both analysis methods (screen $A$ : enhanced $Z$-score rank 21, FDR = 1.26e-19; screen B: enhanced Z-score rank 7, FDR = 5.47e-21). XCL1 (also known as lymphotactin) is the unique ligand for XCR1 and studies with calcium indicators in immune cells have revealed that XCR1 stimulation leads to calcium mobilization (Bachem et al., 2010; Yoshida et al., 1998, 1999). Calcium mobilization most likely results from calcium release from the ER by IP3 receptors upon activation of phospholipase C by XCR1-coupled G-proteins. Thus, it might be plausible that XCR1 contributes to calcium signaling during neuronal activity. Nevertheless, it requires additional investigations to validate this finding.

\subsubsection{Comparison with the screen by Parnas et al.}

Currently the most comprehensive pooled genetic screen has been published by Parnas and colleagues (Parnas et al., 2015). They have used a genome-wide CRISPR-Cas9 knockout library in order to study the response of primary mouse dendritic cells to bacterial LPS. Therefore they sorted the cell pool based on Tnf expression, which is downstream of the LPS/TIr4 pathway. This allowed them to identify many known regulators of the TIr4-to-Tnf pathway. In particular, CRISPR-Cas9-mediated knockout screens are believed to improve screen data quality due to higher on-target efficacy and more pronounced phenotypes compared to shRNA-mediated knockdowns (Evers et al., 2016a; Morgens et al., 2016). Although both screens differ in more aspects besides the screening technology (e.g. cell type, phenotype, readout strategy), some quality control parameters might allow a comparison. As a measure of reproducibility Parnas and colleagues present Pearsoncorrelation coefficients of normalized read counts between two independent screen replicates in the range of $0.45-0.54$. Correlation of replicate samples of the pooled RNAi screen presented in this thesis is in all cases above 0.95 . The difference probably reflects the different readout strategies and might be evidence for the robustness of a RNA barcode sensor. Both screens were analyzed by Z-score ranking and using the DESeq2 package. Parnas and colleagues describe that 50 out of the top 100 positive regulators overlap between the two analysis strategies. The same comparison was done for screen $A$ in this thesis and 41 common genes could be found in the top 100 hit lists from both rankings (see Figure 19C). Thus, by this parameter both screens do not differ a lot. Worth mentioning is that Parnas and colleagues carried out a secondary screen where they used the same cell number as in the primary screen but infected with a 5-fold smaller sgRNA library, focusing on an extended hit list from the primary screen. This further improved the data quality and seems to be a worthwhile strategy for large-scale screens when cell numbers are limiting as in the case of many primary cell types. 


\subsection{Limitations of the approach}

The power of pooled genetic screens lies in the rapid and straightforward identification of genes that modulate a certain phenotype. A pooled genetic screen is, however, not able to resolve temporal or spatial information. These dimensions can at least to some extend be inferred from additional data sources such as pathway and protein localization databases or need to be determined by additional experiments (Fabregat et al., 2016; Ogata et al., 1998; Sprenger et al., 2008). A second general limitation which is inherent to large-scale screening approaches using shRNA libraries is the wide range of shRNA efficacy. Although shRNA design algorithms have improved over the years, it is still necessary to include multiple shRNAs per gene into the library (Kampmann et al., 2015). In particular, with the limited access to primary cells it is thus always a tradeoff between the number of genes included in a screen and the number of shRNAs per gene. Recent findings argue that on-target efficacy of sgRNAs seems to be higher and more predictable which will be beneficial for the next generation of pooled screens in primary neuron cultures (Evers et al., 2016a; Morgens et al., 2016).

In the case of a pooled RNAi screen using the E-SARE sensor under synaptic stimulation it was expected that the screen is blind for the presynapse/axonal compartment. This is because the main signaling route is from the postsynapse along the dendrites to the cell soma and nucleus and via the axon and the presynapse to a connected neuron. Except for neurotrophin-induced signaling endosomes that translocate long distances from the presynapse to the soma, little is known about presynapse-to-nucleus signaling (Fainzilber et al., 2011). A recent study claims that during neuronal activity signaling from the presynapse to the nucleus might exist as well and that it contributes to the regulation of activitydependent gene expression (Ivanova et al., 2015). Ivanova and colleagues found that the nuclear and the presynaptic localization of the co-repressor CtBP1 is interconnected and depends on neuronal activity and presynaptic NAD/NADH levels. In all three screens and by both analysis strategies CtBP1 is a hit as a positive regulator, supporting its function during neuronal activity. Which function CtBP1 exactly fulfills during neuronal excitation and whether the long-distance transport between presynapse and nucleus is really necessary for regulation will require further studies. Nevertheless, it might support findings of presynapseto-nucleus signaling and that components can be identified using the pooled RNAi screen.

\subsection{Future aspects of pooled genetic screens in neuroscience}

This study expands the existing toolbox in functional genomics (RNAi-, CRISPR-Cas9 screens) by a new readout strategy in which pathway activities are monitored by a barcoded RNA reporter. Compared to previous strategies, pathway monitoring by a barcoded reporter 
makes cell sorting dispensable. This broadens the application spectrum and facilitates the screening procedure of cell types with complex morphologies such as primary neurons, where flow cytometry is challenging and unfavorable. The assay has been validated in this proof-of-concept study and is already able to dissect neuronal regulatory networks at so far unprecedented throughput. Screening throughput can still be increased by designing genome-wide next generation libraries. Data quality might be further improved by the adaptation towards a CRISPR-Cas9 screening technology (Evers et al., 2016b). This will in addition provide access to gain-of-function screens (Konermann et al., 2015). The corresponding AAV vector for this approach has been presented and preliminarily validated in this thesis.

As emphasized earlier, several genes with associations to psychiatric diseases have been identified by the pooled RNAi screen. The majority of common variants in psychiatric patients that have been discovered by GWAS analyses seem to lie in regulatory elements (e.g. enhancers, core promoters) (Maurano et al., 2012). Usually the most proximal gene is reported as a potential risk gene although empirical validation is missing in most cases. Mutations in enhancer and promoter regions can result in up- and down-regulation of the corresponding gene. A complementary screening approach using CRISPR-Cas9-mediated gain-of-function and shRNA- or CRISPR-Cas9-mediated loss-of-function might facilitate and accelerate the empirical identification of risk genes at genome-wide GWAS loci.

\subsubsection{Importance for psychiatric drug discovery}

The pharmaceutical industry has experienced reduced productivity and increasing costs for first-in-class drug discovery over the last decades (Scannell et al., 2012). Main reasons are high attrition rates for drugs during clinical trials due to lack of efficacy and safety issues. This repression is particularly visible in psychiatric drug discovery where for example most of the current medications for schizophrenia have been discovered decades ago (Agid et al., 2007; Kapur and Mamo, 2003; Papassotiropoulos and de Quervain, 2015). The lack of a primary target in many psychiatric disorders has made target-based drug discovery attempts using biochemical binding assays highly inefficient. In the last years, however, hope is emerging from the progress made with phenotypic screenings (Haggarty et al., 2016; Kaiser and Feng, 2015). Phenotypic screening describes in the best case the testing of compounds using a cellular or even in vivo model of the disease, under physiological conditions and with a readout that has translational validity into the clinic (Vincent et al., 2015). The increased biological relevance of the approach is believed to enhance the predictive validity and it expands the biological target space (Scannell and Bosley, 2016). Consequently, the target of a hit compound identified by phenotypic screenings is usually unknown. A recent survey on 
how new medications were discovered revealed that between 1999 and 2008 FDA-approved first-in-class drugs mainly originated from phenotypic screenings (Swinney and Anthony, 2011). In the field of CNS diseases 7 out of 9 new molecular entities (NME) came from phenotypic drug discovery and many still do not have an identified target or MoA.

Genetic interference screens (RNAi, CRISPR-Cas9, arrayed or pooled) are already part of the standard repertoire for target identification during drug discovery in oncology, for example (Fennell et al., 2014; Schenone et al., 2013). Thus, the presented assay delivers the missing tool for target identification in phenotypic drug discovery for CNS diseases. As elaborated earlier functional genomics is currently experiencing a transformation from RNAi towards CRISPR-Cas9 knockout screens. However, it should not been forgotten that both screening technologies have their unique properties (Deans et al., 2016). Knockdown of a drug target using shRNAs is expected to phenocopy the inhibitory action of the drug on that target. The incomplete knockdown efficiency of shRNAs, a frequently criticized feature, allows the study of epistatic interactions between shRNA-mediated knockdown and drugmediated inhibition of a protein. The power of both systems has been recently compared and used for target identification. This study highlighted that shRNA-mediated knockdown is well suited for the identification of essential genes for a given phenotype, whereas CRISPRCas9-mediated knockout is advantageous for the discovery of non-essential genes (Deans et al., 2016; Morgens et al., 2016). 


\section{Abbreviations}

\begin{tabular}{|c|c|}
\hline AAV & Adeno-associated virus \\
\hline AD & Alzheimer disease \\
\hline Adcy & Adenylate cyclase \\
\hline AMPA & a-amino-3-hydroxy-5-methyl-4-isoxazole propionic acid \\
\hline ASD & Autism spectrum disorder \\
\hline $\mathrm{BC}$ & Barcode \\
\hline BDNF & Brain-derived neurotrophic factor \\
\hline $\mathrm{BIC}$ & Bicuculline \\
\hline BPD & Bipolar disorder \\
\hline CAMP & 3',5'-cyclic adenosine monophosphate \\
\hline CNS & Central nervous system \\
\hline CNV & Copy number variation \\
\hline CRISPR & Clustered regularly interspaced short palindromic repeats \\
\hline crRNA & CRISPR RNA \\
\hline DAG & Diacylglycerol \\
\hline DIV & Day in vitro \\
\hline $\mathrm{E} / \mathrm{I}$ & Excitation-inhibition balance \\
\hline ER & Endoplasmic reticulum \\
\hline FACS & Fluorescence-activated cell sorting \\
\hline FBS & Fetal bovine serum \\
\hline FDR & False discovery rate \\
\hline GPCR & G-protein coupled receptor \\
\hline GWAS & Genome-wide association study \\
\hline HS & Horse serum \\
\hline IEG & Immediate early gene \\
\hline IP3 & Inositol-1,4,5-trisphosphate \\
\hline iPSC & Induced pluripotent stem cell \\
\hline LPS & Lipopolysaccharide \\
\hline LTP/LTD & Long-term potentiation/depression \\
\hline MAPK & Mitogen-activated protein kinase \\
\hline MDD & Major depressive disorder \\
\hline MoA & Mode of action \\
\hline MPRA & Massively parallel reporter gene assay \\
\hline NGS & Next-generation sequencing \\
\hline NME & New molecular entity \\
\hline NMDA & $\mathrm{N}$-methyl-D-aspartate \\
\hline PAM & Proximal adjacent motif \\
\hline
\end{tabular}




$\begin{array}{ll}\text { PGC } & \text { Psychiatric Genomics Consortium } \\ \text { PMA } & \text { Phorbol-12-myristat-13-acetat } \\ \text { PSD } & \text { Post-synaptic density } \\ \text { PV } & \text { Parv-albumin } \\ \text { RNAi } & \text { RNA interference } \\ \text { sd } & \text { Standard deviation } \\ \text { sem } & \text { Standard error of the mean } \\ \text { sgRNA } & \text { Short guide RNA } \\ \text { shRNA } & \text { Short hairpin RNA } \\ \text { siRNA } & \text { Small interfering RNA } \\ \text { SV } & \text { Structural variant } \\ \text { SV40 } & \text { Simian virus 40 } \\ \text { tracrRNA } & \text { Trans-activating crRNA } \\ \text { VGCC } & \text { Voltage-gated calcium channel }\end{array}$




\section{References}

Abbott, L.F., and Regehr, W.G. (2004). Synaptic computation. Nature 431, 796-803.

Adams, J.P., and Dudek, S.M. (2005). Late-phase long-term potentiation: getting to the nucleus. Nat. Rev. Neurosci. 6, 737-743.

Agid, Y., Buzsáki, G., Diamond, D.M., Frackowiak, R., Giedd, J., Girault, J.-A., Grace, A., Lambert, J.J., Manji, H., Mayberg, H., et al. (2007). How can drug discovery for psychiatric disorders be improved? Nat. Rev. Drug Discov. 6, 189-201.

Amtul, Z., and Atta-Ur-Rahman, null (2015). Neural plasticity and memory: molecular mechanism. Rev. Neurosci. 26, 253-268.

Anderl, J.L., Redpath, S., and Ball, A.J. (2009). A neuronal and astrocyte co-culture assay for high content analysis of neurotoxicity. J. Vis. Exp. JoVE.

Arthur, J.S.C., Fong, A.L., Dwyer, J.M., Davare, M., Reese, E., Obrietan, K., and Impey, S. (2004). Mitogen- and stress-activated protein kinase 1 mediates cAMP response elementbinding protein phosphorylation and activation by neurotrophins. J. Neurosci. Off. J. Soc. Neurosci. 24, 4324-4332.

Azevedo, F.A.C., Carvalho, L.R.B., Grinberg, L.T., Farfel, J.M., Ferretti, R.E.L., Leite, R.E.P., Jacob Filho, W., Lent, R., and Herculano-Houzel, S. (2009). Equal numbers of neuronal and nonneuronal cells make the human brain an isometrically scaled-up primate brain. J. Comp. Neurol. 513, 532-541.

Bachelerie, F., Ben-Baruch, A., Burkhardt, A.M., Combadiere, C., Farber, J.M., Graham, G.J., Horuk, R., Sparre-Ulrich, A.H., Locati, M., Luster, A.D., et al. (2014). International Union of Basic and Clinical Pharmacology. [corrected]. LXXXIX. Update on the extended family of chemokine receptors and introducing a new nomenclature for atypical chemokine receptors. Pharmacol. Rev. 66, 1-79.

Bachem, A., Güttler, S., Hartung, E., Ebstein, F., Schaefer, M., Tannert, A., Salama, A., Movassaghi, K., Opitz, C., Mages, H.W., et al. (2010). Superior antigen cross-presentation and XCR1 expression define human CD11C+CD141+ cells as homologues of mouse CD8+ dendritic cells. J. Exp. Med. 207, 1273-1281.

Bading, H. (2013). Nuclear calcium signalling in the regulation of brain function. Nat. Rev. Neurosci. 14, 593-608.

Bading, H., Hardingham, G.E., Johnson, C.M., and Chawla, S. (1997). Gene regulation by nuclear and cytoplasmic calcium signals. Biochem. Biophys. Res. Commun. 236, 541-543.

Baj, G., Patrizio, A., Montalbano, A., Sciancalepore, M., and Tongiorgi, E. (2014). Developmental and maintenance defects in Rett syndrome neurons identified by a new mouse staging system in vitro. Front. Cell. Neurosci. 8, 18.

Balkwill, F. (1998). The molecular and cellular biology of the chemokines. J. Viral Hepat. 5, $1-14$.

Barco, A., Patterson, S.L., Patterson, S., Alarcon, J.M., Gromova, P., Mata-Roig, M., Morozov, A., and Kandel, E.R. (2005). Gene expression profiling of facilitated L-LTP in VP16-CREB mice reveals that BDNF is critical for the maintenance of LTP and its synaptic capture. Neuron 48, 123-137. 
Bartos, M., Vida, I., and Jonas, P. (2007). Synaptic mechanisms of synchronized gamma oscillations in inhibitory interneuron networks. Nat. Rev. Neurosci. 8, 45-56.

Bassett, A.S., Marshall, C.R., Lionel, A.C., Chow, E.W.C., and Scherer, S.W. (2008). Copy number variations and risk for schizophrenia in 22q11.2 deletion syndrome. Hum. Mol. Genet. 17, 4045-4053.

Bassik, M.C., Lebbink, R.J., Churchman, L.S., Ingolia, N.T., Patena, W., LeProust, E.M., Schuldiner, M., Weissman, J.S., and McManus, M.T. (2009). Rapid Creation and Quantitative Monitoring of High Coverage shRNA Libraries. Nat. Methods 6, 443-445.

Bassik, M.C., Kampmann, M., Lebbink, R.J., Wang, S., Hein, M.Y., Poser, I., Weibezahn, J., Horlbeck, M.A., Chen, S., Mann, M., et al. (2013). A systematic mammalian genetic interaction map reveals pathways underlying ricin susceptibility. Cell 152, 909-922.

Bayés, A., van de Lagemaat, L.N., Collins, M.O., Croning, M.D.R., Whittle, I.R., Choudhary, J.S., and Grant, S.G.N. (2011). Characterization of the proteome, diseases and evolution of the human postsynaptic density. Nat. Neurosci. 14, 19-21.

Bengtson, C.P., Freitag, H.E., Weislogel, J.-M., and Bading, H. (2010). Nuclear calcium sensors reveal that repetition of trains of synaptic stimuli boosts nuclear calcium signaling in CA1 pyramidal neurons. Biophys. J. 99, 4066-4077.

Benjamini, Y., and Hochberg, Y. (1995). Controlling the False Discovery Rate: A Practical and Powerful Approach to Multiple Testing. J. R. Stat. Soc. Ser. B Methodol. 57, 289-300.

Benke, T.A., Lüthi, A., Isaac, J.T., and Collingridge, G.L. (1998). Modulation of AMPA receptor unitary conductance by synaptic activity. Nature 393, 793-797.

Berezovska, O., McLean, P., Knowles, R., Frosh, M., Lu, F.M., Lux, S.E., and Hyman, B.T. (1999). Notch1 inhibits neurite outgrowth in postmitotic primary neurons. Neuroscience 93, 433-439.

Berridge, M.J. (1998). Neuronal calcium signaling. Neuron 21, 13-26.

Bertollini, C., Ragozzino, D., Gross, C., Limatola, C., and Eusebi, F. (2006). Fractalkine/CX3CL1 depresses central synaptic transmission in mouse hippocampal slices. Neuropharmacology 51, 816-821.

Birmingham, A., Selfors, L.M., Forster, T., Wrobel, D., Kennedy, C.J., Shanks, E., SantoyoLopez, J., Dunican, D.J., Long, A., Kelleher, D., et al. (2009). Statistical Methods for Analysis of High-Throughput RNA Interference Screens. Nat. Methods 6, 569-575.

Black, J.E., Kodish, I.M., Grossman, A.W., Klintsova, A.Y., Orlovskaya, D., Vostrikov, V., Uranova, N., and Greenough, W.T. (2004). Pathology of layer V pyramidal neurons in the prefrontal cortex of patients with schizophrenia. Am. J. Psychiatry 161, 742-744.

Borchert, G.M., Lanier, W., and Davidson, B.L. (2006). RNA polymerase III transcribes human microRNAs. Nat. Struct. Mol. Biol. 13, 1097-1101.

Botvinnik, A., Wichert, S.P., Fischer, T.M., and Rossner, M.J. (2010). Integrated analysis of receptor activation and downstream signaling with EXTassays. Nat Meth 7, 74-80.

Bouard, D., Alazard-Dany, N., and Cosset, F.-L. (2009). Viral vectors: from virology to transgene expression. Br. J. Pharmacol. 157, 153-165. 
Boutros, M., Heigwer, F., and Laufer, C. (2015). Microscopy-Based High-Content Screening. Cell 163, 1314-1325.

Brami-Cherrier, K., Lavaur, J., Pagès, C., Arthur, J.S.C., and Caboche, J. (2007). Glutamate induces histone $\mathrm{H} 3$ phosphorylation but not acetylation in striatal neurons: role of mitogenand stress-activated kinase-1. J. Neurochem. 101, 697-708.

Brennand, K.J., Simone, A., Jou, J., Gelboin-Burkhart, C., Tran, N., Sangar, S., Li, Y., Mu, Y., Chen, G., Yu, D., et al. (2011). Modelling schizophrenia using human induced pluripotent stem cells. Nature 473, 221-225.

Brincat, S.L., and Miller, E.K. (2015). Frequency-specific hippocampal-prefrontal interactions during associative learning. Nat. Neurosci. 18, 576-581.

Bullmore, E., and Sporns, O. (2009). Complex brain networks: graph theoretical analysis of structural and functional systems. Nat. Rev. Neurosci. 10, 186-198.

Caicedo, J.C., Singh, S., and Carpenter, A.E. (2016). Applications in image-based profiling of perturbations. Curr. Opin. Biotechnol. 39, 134-142.

Campbell, F.E., and Setzer, D.R. (1992). Transcription termination by RNA polymerase III: uncoupling of polymerase release from termination signal recognition. Mol. Cell. Biol. 12, 2260-2272.

Carthew, R.W., and Sontheimer, E.J. (2009). Origins and Mechanisms of miRNAs and siRNAs. Cell 136, 642-655.

Charles A Janeway, J., Travers, P., Walport, M., and Shlomchik, M.J. (2001). The major histocompatibility complex and its functions.

Chawla, S. (2002). Regulation of gene expression by Ca2+ signals in neuronal cells. Eur. J. Pharmacol. 447, 131-140.

Chawla, S., Hardingham, G.E., Quinn, D.R., and Bading, H. (1998). CBP: a signal-regulated transcriptional coactivator controlled by nuclear calcium and CaM kinase IV. Science 281, 1505-1509.

Chendrimada, T.P., Gregory, R.I., Kumaraswamy, E., Norman, J., Cooch, N., Nishikura, K., and Shiekhattar, R. (2005). TRBP recruits the Dicer complex to Ago2 for microRNA processing and gene silencing. Nature 436, 740-744.

Chowdhury, S., Shepherd, J.D., Okuno, H., Lyford, G., Petralia, R.S., Plath, N., Kuhl, D., Huganir, R.L., and Worley, P.F. (2006). Arc/Arg3.1 interacts with the endocytic machinery to regulate AMPA receptor trafficking. Neuron 52, 445-459.

Citri, A., and Malenka, R.C. (2008). Synaptic plasticity: multiple forms, functions, and mechanisms. Neuropsychopharmacol. Off. Publ. Am. Coll. Neuropsychopharmacol. 33, 1841.

Collins, S.R., Weissman, J.S., and Krogan, N.J. (2009). From information to knowledge: new technologies for defining gene function. Nat. Methods 6, 721-723.

Cross-Disorder Group of the Psychiatric Genomics Consortium, Lee, S.H., Ripke, S., Neale, B.M., Faraone, S.V., Purcell, S.M., Perlis, R.H., Mowry, B.J., Thapar, A., Goddard, M.E., et al. (2013). Genetic relationship between five psychiatric disorders estimated from genomewide SNPs. Nat. Genet. 45, 984-994. 
Curtis, D.R., Duggan, A.W., Felix, D., and Johnston, G.A. (1970). GABA, bicuculline and central inhibition. Nature 226, 1222-1224.

Dai, Z., Sheridan, J.M., Gearing, L.J., Moore, D.L., Su, S., Wormald, S., Wilcox, S., O'Connor, L., Dickins, R.A., Blewitt, M.E., et al. (2014). edgeR: a versatile tool for the analysis of shRNA-seq and CRISPR-Cas9 genetic screens. F1000Research 3, 95.

Darnell, J.C., Van Driesche, S.J., Zhang, C., Hung, K.Y.S., Mele, A., Fraser, C.E., Stone, E.F., Chen, C., Fak, J.J., Chi, S.W., et al. (2011). FMRP Stalls Ribosomal Translocation on mRNAs Linked to Synaptic Function and Autism. Cell 146, 247-261.

Das, G., Henning, D., Wright, D., and Reddy, R. (1988). Upstream regulatory elements are necessary and sufficient for transcription of a U6 RNA gene by RNA polymerase III. EMBO J. 7, 503-512.

Deans, R.M., Morgens, D.W., Ökesli, A., Pillay, S., Horlbeck, M.A., Kampmann, M., Gilbert, L.A., Li, A., Mateo, R., Smith, M., et al. (2016). Parallel shRNA and CRISPR-Cas9 screens enable antiviral drug target identification. Nat. Chem. Biol. 12, 361-366.

De Strooper, B. (2007). Loss-of-function presenilin mutations in Alzheimer disease. Talking Point on the role of presenilin mutations in Alzheimer disease. EMBO Rep. 8, 141-146.

Deltcheva, E., Chylinski, K., Sharma, C.M., Gonzales, K., Chao, Y., Pirzada, Z.A., Eckert, M.R., Vogel, J., and Charpentier, E. (2011). CRISPR RNA maturation by trans-encoded small RNA and host factor RNase III. Nature 471, 602-607.

Diehl, P., Tedesco, D., and Chenchik, A. (2014). Use of RNAi screens to uncover resistance mechanisms in cancer cells and identify synthetic lethal interactions. Drug Discov. Today Technol. 11, 11-18.

Dimos, J.T., Rodolfa, K.T., Niakan, K.K., Weisenthal, L.M., Mitsumoto, H., Chung, W., Croft, G.F., Saphier, G., Leibel, R., Goland, R., et al. (2008). Induced pluripotent stem cells generated from patients with ALS can be differentiated into motor neurons. Science 321, 1218-1221.

Dobrunz, L.E., and Stevens, C.F. (1997). Heterogeneity of release probability, facilitation, and depletion at central synapses. Neuron 18, 995-1008.

Dolmetsch, R. (2003). Excitation-transcription coupling: signaling by ion channels to the nucleus. Sci. STKE Signal Transduct. Knowl. Environ. 2003, PE4.

Dolmetsch, R.E., Pajvani, U., Fife, K., Spotts, J.M., and Greenberg, M.E. (2001). Signaling to the nucleus by an L-type calcium channel-calmodulin complex through the MAP kinase pathway. Science 294, 333-339.

Ebert, D.H., and Greenberg, M.E. (2013). Activity-dependent neuronal signalling and autism spectrum disorder. Nature 493, 327-337.

Ebinu, J.O., Bottorff, D.A., Chan, E.Y., Stang, S.L., Dunn, R.J., and Stone, J.C. (1998). RasGRP, a Ras guanyl nucleotide- releasing protein with calcium- and diacylglycerol-binding motifs. Science 280, 1082-1086.

Emamian, E.S., Hall, D., Birnbaum, M.J., Karayiorgou, M., and Gogos, J.A. (2004). Convergent evidence for impaired AKT1-GSK3beta signaling in schizophrenia. Nat. Genet. 36, 131-137. 
Engelman, H.S., and MacDermott, A.B. (2004). Presynaptic ionotropic receptors and control of transmitter release. Nat. Rev. Neurosci. 5, 135-145.

Evers, B., Jastrzebski, K., Heijmans, J.P.M., Grernrum, W., Beijersbergen, R.L., and Bernards, R. (2016a). CRISPR knockout screening outperforms shRNA and CRISPRi in identifying essential genes. Nat. Biotechnol.

Evers, B., Jastrzebski, K., Heijmans, J.P.M., Grernrum, W., Beijersbergen, R.L., and Bernards, R. (2016b). CRISPR knockout screening outperforms shRNA and CRISPRi in identifying essential genes. Nat. Biotechnol.

Fabregat, A., Sidiropoulos, K., Garapati, P., Gillespie, M., Hausmann, K., Haw, R., Jassal, B., Jupe, S., Korninger, F., McKay, S., et al. (2016). The Reactome pathway Knowledgebase. Nucleic Acids Res. 44, D481-487.

Fainzilber, M., Budnik, V., Segal, R.A., and Kreutz, M.R. (2011). From synapse to nucleus and back again--communication over distance within neurons. J. Neurosci. Off. J. Soc. Neurosci. 31, 16045-16048.

Farnsworth, C.L., Freshney, N.W., Rosen, L.B., Ghosh, A., Greenberg, M.E., and Feig, L.A. (1995). Calcium activation of Ras mediated by neuronal exchange factor Ras-GRF. Nature 376, 524-527.

Farr, C.D., Gafken, P.R., Norbeck, A.D., Doneanu, C.E., Stapels, M.D., Barofsky, D.F., Minami, M., and Saugstad, J.A. (2004). Proteomic analysis of native metabotropic glutamate receptor 5 protein complexes reveals novel molecular constituents. J. Neurochem. 91, 438450 .

Fatt, P., and Katz, B. (1953). The electrical properties of crustacean muscle fibres. J. Physiol. 120, 171-204.

Fennell, M., Xiang, Q., Hwang, A., Chen, C., Huang, C.-H., Chen, C.-C., Pelossof, R., and Garippa, R.J. (2014). Impact of RNA-guided technologies for target identification and deconvolution. J. Biomol. Screen. 19, 1327-1337.

Filipowicz, W., Bhattacharyya, S.N., and Sonenberg, N. (2008). Mechanisms of posttranscriptional regulation by microRNAs: are the answers in sight? Nat. Rev. Genet. 9, 102114.

Fire, A., Xu, S., Montgomery, M.K., Kostas, S.A., Driver, S.E., and Mello, C.C. (1998). Potent and specific genetic interference by double-stranded RNA in Caenorhabditis elegans. Nature 391, 806-811.

Flavell, S.W., Cowan, C.W., Kim, T.-K., Greer, P.L., Lin, Y., Paradis, S., Griffith, E.C., Hu, L.S., Chen, C., and Greenberg, M.E. (2006). Activity-dependent regulation of MEF2 transcription factors suppresses excitatory synapse number. Science 311, 1008-1012.

Forrest, M.D. (2014). The sodium-potassium pump is an information processing element in brain computation. Front. Physiol. 5, 472.

Frank, F., Sonenberg, N., and Nagar, B. (2010). Structural basis for 5'-nucleotide basespecific recognition of guide RNA by human AGO2. Nature 465, 818-822.

Franke, B., Stein, J.L., Ripke, S., Anttila, V., Hibar, D.P., van Hulzen, K.J.E., Arias-Vasquez, A., Smoller, J.W., Nichols, T.E., Neale, M.C., et al. (2016). Genetic influences on schizophrenia and subcortical brain volumes: large-scale proof of concept. Nat. Neurosci. 19, 420-431. 
Freedman, L.P., Cockburn, I.M., and Simcoe, T.S. (2015). The Economics of Reproducibility in Preclinical Research. PLoS Biol. 13, e1002165.

Fromer, M., Pocklington, A.J., Kavanagh, D.H., Williams, H.J., Dwyer, S., Gormley, P., Georgieva, L., Rees, E., Palta, P., Ruderfer, D.M., et al. (2014). De novo mutations in schizophrenia implicate synaptic networks. Nature 506, 179-184.

Garner, A.R., Rowland, D.C., Hwang, S.Y., Baumgaertel, K., Roth, B.L., Kentros, C., and Mayford, M. (2012). Generation of a synthetic memory trace. Science 335, 1513-1516.

Geschwind, D.H., and Flint, J. (2015). Genetics and genomics of psychiatric disease. Science 349, 1489-1494.

Giaever, G., Shoemaker, D.D., Jones, T.W., Liang, H., Winzeler, E.A., Astromoff, A., and Davis, R.W. (1999). Genomic profiling of drug sensitivities via induced haploinsufficiency. Nat Genet 21, 278-283.

Giaever, G., Chu, A.M., Ni, L., Connelly, C., Riles, L., Veronneau, S., Dow, S., Lucau-Danila, A., Anderson, K., Andre, B., et al. (2002). Functional profiling of the Saccharomyces cerevisiae genome. Nature 418, 387-391.

Gilbert, L.A., Horlbeck, M.A., Adamson, B., Villalta, J.E., Chen, Y., Whitehead, E.H., Guimaraes, C., Panning, B., Ploegh, H.L., Bassik, M.C., et al. (2014). Genome-Scale CRISPR-Mediated Control of Gene Repression and Activation. Cell.

Gille, H., Kortenjann, M., Thomae, O., Moomaw, C., Slaughter, C., Cobb, M.H., and Shaw, P.E. (1995). ERK phosphorylation potentiates Elk-1-mediated ternary complex formation and transactivation. EMBO J. 14, 951-962.

Giovannelli, A., Limatola, C., Ragozzino, D., Mileo, A.M., Ruggieri, A., Ciotti, M.T., Mercanti, D., Santoni, A., and Eusebi, F. (1998). CXC chemokines interleukin-8 (IL-8) and growthrelated gene product alpha (GROalpha) modulate Purkinje neuron activity in mouse cerebellum. J. Neuroimmunol. 92, 122-132.

Grant, S.G.N. (2012). Synaptopathies: diseases of the synaptome. Curr. Opin. Neurobiol. 22, 522-529.

Greenberg, M.E., Greene, L.A., and Ziff, E.B. (1985). Nerve growth factor and epidermal growth factor induce rapid transient changes in proto-oncogene transcription in PC12 cells. J. Biol. Chem. 260, 14101-14110.

Greer, P.L., and Greenberg, M.E. (2008). From synapse to nucleus: calcium-dependent gene transcription in the control of synapse development and function. Neuron 59, 846-860.

Gregory, R.I., Chendrimada, T.P., Cooch, N., and Shiekhattar, R. (2005). Human RISC Couples MicroRNA Biogenesis and Posttranscriptional Gene Silencing. Cell 123, 631-640.

Gu, X.Q., Yao, H., and Haddad, G.G. (2001). Increased neuronal excitability and seizures in the $\mathrm{Na}(+) / H(+)$ exchanger null mutant mouse. Am. J. Physiol. Cell Physiol. 281, C496-503.

Guidotti, A., Auta, J., Davis, J.M., Di-Giorgi-Gerevini, V., Dwivedi, Y., Grayson, D.R., Impagnatiello, F., Pandey, G., Pesold, C., Sharma, R., et al. (2000). Decrease in reelin and glutamic acid decarboxylase67 (GAD67) expression in schizophrenia and bipolar disorder: a postmortem brain study. Arch. Gen. Psychiatry 57, 1061-1069. 
Guyon, A., Skrzydelsi, D., Rovère, C., Rostène, W., Parsadaniantz, S.M., and Nahon, J.L. (2006). Stromal cell-derived factor-1alpha modulation of the excitability of rat substantia nigra dopaminergic neurones: presynaptic mechanisms. J. Neurochem. 96, 1540-1550.

de Haas, A.H., van Weering, H.R.J., de Jong, E.K., Boddeke, H.W.G.M., and Biber, K.P.H. (2007). Neuronal Chemokines: Versatile Messengers In Central Nervous System Cell Interaction. Mol. Neurobiol. 36, 137-151.

Habela, C.W., Song, H., and Ming, G.-L. (2016). Modeling synaptogenesis in schizophrenia and autism using human iPSC derived neurons. Mol. Cell. Neurosci. 73, 52-62.

Haenschel, C., Bittner, R.A., Waltz, J., Haertling, F., Wibral, M., Singer, W., Linden, D.E.J., and Rodriguez, E. (2009). Cortical oscillatory activity is critical for working memory as revealed by deficits in early-onset schizophrenia. J. Neurosci. Off. J. Soc. Neurosci. 29, 9481-9489.

Haggarty, S.J., Silva, M.C., Cross, A., Brandon, N.J., and Perlis, R.H. (2016). Advancing drug discovery for neuropsychiatric disorders using patient-specific stem cell models. Mol. Cell. Neurosci.

Hardingham, G.E., Chawla, S., Johnson, C.M., and Bading, H. (1997). Distinct functions of nuclear and cytoplasmic calcium in the control of gene expression. Nature 385, 260-265.

Hardingham, G.E., Arnold, F.J., and Bading, H. (2001). A calcium microdomain near NMDA receptors: on switch for ERK-dependent synapse-to-nucleus communication. Nat. Neurosci. $4,565-566$.

Harrill, J.A., Robinette, B.L., and Mundy, W.R. (2011). Use of high content image analysis to detect chemical-induced changes in synaptogenesis in vitro. Toxicol. Vitro Int. J. Publ. Assoc. BIBRA 25, 368-387.

Hartman, J.J., Mahr, J., McNally, K., Okawa, K., Iwamatsu, A., Thomas, S., Cheesman, S., Heuser, J., Vale, R.D., and McNally, F.J. (1998). Katanin, a microtubule-severing protein, is a novel AAA ATPase that targets to the centrosome using a WD40-containing subunit. Cell 93, 277-287.

Henley, J.M., and Wilkinson, K.A. (2016). Synaptic AMPA receptor composition in development, plasticity and disease. Nat. Rev. Neurosci.

Hensch, T.K. (2005). Critical period plasticity in local cortical circuits. Nat. Rev. Neurosci. 6, 877-888.

Hensel, M., Shea, J., Gleeson, C., Jones, M., Dalton, E., and Holden, D. (1995). Simultaneous identification of bacterial virulence genes by negative selection. Science 269, 400-403.

Homayoun, H., and Moghaddam, B. (2007a). Fine-tuning of awake prefrontal cortex neurons by clozapine: comparison with haloperidol and N-desmethylclozapine. Biol. Psychiatry 61, 679-687.

Homayoun, H., and Moghaddam, B. (2007b). NMDA receptor hypofunction produces opposite effects on prefrontal cortex interneurons and pyramidal neurons. J. Neurosci. Off. J. Soc. Neurosci. 27, 11496-11500.

Hong, L.E., Summerfelt, A., Buchanan, R.W., O'Donnell, P., Thaker, G.K., Weiler, M.A., and Lahti, A.C. (2010). Gamma and delta neural oscillations and association with clinical 
symptoms under subanesthetic ketamine. Neuropsychopharmacol. Off. Publ. Am. Coll. Neuropsychopharmacol. 35, 632-640.

Hsu, P.D., Lander, E.S., and Zhang, F. (2014). Development and Applications of CRISPRCas9 for Genome Engineering. Cell 157, 1262-1278.

Hunsberger, J.G., Efthymiou, A.G., Malik, N., Behl, M., Mead, I.L., Zeng, X., Simeonov, A., and Rao, M. (2015). Induced Pluripotent Stem Cell Models to Enable In Vitro Models for Screening in the Central Nervous System. Stem Cells Dev. 24, 1852-1864.

Igarashi, K.M. (2015). Plasticity in oscillatory coupling between hippocampus and cortex. Curr. Opin. Neurobiol. 35, 163-168.

Impey, S., Fong, A.L., Wang, Y., Cardinaux, J.R., Fass, D.M., Obrietan, K., Wayman, G.A., Storm, D.R., Soderling, T.R., and Goodman, R.H. (2002). Phosphorylation of CBP mediates transcriptional activation by neural activity and CaM kinase IV. Neuron 34, 235-244.

Ivanova, D., Dirks, A., Montenegro-Venegas, C., Schöne, C., Altrock, W.D., Marini, C., Frischknecht, R., Schanze, D., Zenker, M., Gundelfinger, E.D., et al. (2015). Synaptic activity controls localization and function of CtBP1 via binding to Bassoon and Piccolo. EMBO J. 34, 1056-1077.

Jaffe, D.B., and Brown, T.H. (1994). Metabotropic glutamate receptor activation induces calcium waves within hippocampal dendrites. J. Neurophysiol. 72, 471-474.

Jeyabalan, N., and Clement, J.P. (2016). SYNGAP1: Mind the Gap. Front. Cell. Neurosci. $10,32$.

Jinek, M., Chylinski, K., Fonfara, I., Hauer, M., Doudna, J.A., and Charpentier, E. (2012). A programmable dual-RNA-guided DNA endonuclease in adaptive bacterial immunity. Science $337,816-821$.

Jinek, M., East, A., Cheng, A., Lin, S., Ma, E., and Doudna, J. (2013). RNA-programmed genome editing in human cells. eLife 2, e00471.

Kaiser, T., and Feng, G. (2015). Modeling psychiatric disorders for developing effective treatments. Nat. Med. 21, 979-988.

Kampmann, M., Horlbeck, M.A., Chen, Y., Tsai, J.C., Bassik, M.C., Gilbert, L.A., Villalta, J.E., Kwon, S.C., Chang, H., Kim, V.N., et al. (2015). Next-generation libraries for robust RNA interference-based genome-wide screens. Proc. Natl. Acad. Sci. U. S. A. 112, E3384E3391.

Kandel, E.R. (2001). The Molecular Biology of Memory Storage: A Dialogue Between Genes and Synapses. Science 294, 1030-1038.

Kapur, S., and Mamo, D. (2003). Half a century of antipsychotics and still a central role for dopamine D2 receptors. Prog. Neuropsychopharmacol. Biol. Psychiatry 27, 1081-1090.

Karayiorgou, M., Simon, T.J., and Gogos, J.A. (2010). 22q11.2 microdeletions: linking DNA structural variation to brain dysfunction and schizophrenia. Nat. Rev. Neurosci. 11, 402-416.

Kawashima, T., Okuno, H., Nonaka, M., Adachi-Morishima, A., Kyo, N., Okamura, M., Takemoto-Kimura, S., Worley, P.F., and Bito, H. (2009). Synaptic activity-responsive element in the Arc/Arg3.1 promoter essential for synapse-to-nucleus signaling in activated neurons. Proc. Natl. Acad. Sci. U. S. A. 106, 316-321. 
Kawashima, T., Kitamura, K., Suzuki, K., Nonaka, M., Kamijo, S., Takemoto-Kimura, S., Kano, M., Okuno, H., Ohki, K., and Bito, H. (2013). Functional labeling of neurons and their projections using the synthetic activity-dependent promoter E-SARE. Nat. Methods 10, 889895.

Khvorova, A., Reynolds, A., and Jayasena, S.D. (2003). Functional siRNAs and miRNAs exhibit strand bias. Cell 115, 209-216.

Kim, J.Y., Duan, X., Liu, C.Y., Jang, M.-H., Guo, J.U., Pow-anpongkul, N., Kang, E., Song, H., and Ming, G. (2009). DISC1 regulates new neuron development in the adult brain via modulation of AKT-mTOR signaling through KIAA1212. Neuron 63, 761-773.

Kim, M.J., Dunah, A.W., Wang, Y.T., and Sheng, M. (2005). Differential roles of NR2A- and NR2B-containing NMDA receptors in Ras-ERK signaling and AMPA receptor trafficking. Neuron 46, 745-760.

Kim, T.-K., Hemberg, M., Gray, J.M., Costa, A.M., Bear, D.M., Wu, J., Harmin, D.A., Laptewicz, M., Barbara-Haley, K., Kuersten, S., et al. (2010). Widespread transcription at neuronal activity-regulated enhancers. Nature 465, 182-187.

Kirov, G., Pocklington, A.J., Holmans, P., Ivanov, D., Ikeda, M., Ruderfer, D., Moran, J., Chambert, K., Toncheva, D., Georgieva, L., et al. (2012). De novo CNV analysis implicates specific abnormalities of postsynaptic signalling complexes in the pathogenesis of schizophrenia. Mol. Psychiatry 17, 142-153.

Kolomeets, N.S., Orlovskaya, D.D., Rachmanova, V.I., and Uranova, N.A. (2005). Ultrastructural alterations in hippocampal mossy fiber synapses in schizophrenia: a postmortem morphometric study. Synap. N. Y. N 57, 47-55.

Kombian, S.B., Mouginot, D., and Pittman, Q.J. (1997). Dendritically released peptides act as retrograde modulators of afferent excitation in the supraoptic nucleus in vitro. Neuron 19 , 903-912.

Konermann, S., Brigham, M.D., Trevino, A.E., Joung, J., Abudayyeh, O.O., Barcena, C., Hsu, P.D., Habib, N., Gootenberg, J.S., Nishimasu, H., et al. (2015). Genome-scale transcriptional activation by an engineered CRISPR-Cas 9 complex. Nature 517, 583-588.

Krey, J.F., Paşca, S.P., Shcheglovitov, A., Yazawa, M., Schwemberger, R., Rasmusson, R., and Dolmetsch, R.E. (2013). Timothy syndrome is associated with activity-dependent dendritic retraction in rodent and human neurons. Nat. Neurosci. 16, 201-209.

Kuwajima, G., Futatsugi, A., Niinobe, M., Nakanishi, S., and Mikoshiba, K. (1992). Two types of ryanodine receptors in mouse brain: skeletal muscle type exclusively in Purkinje cells and cardiac muscle type in various neurons. Neuron 9, 1133-1142.

Larson, M.H., Gilbert, L.A., Wang, X., Lim, W.A., Weissman, J.S., and Qi, L.S. (2013). CRISPR interference (CRISPRi) for sequence-specific control of gene expression. Nat. Protoc. 8, 2180-2196.

Lee, Y., Ahn, C., Han, J., Choi, H., Kim, J., Yim, J., Lee, J., Provost, P., Rådmark, O., Kim, S., et al. (2003). The nuclear RNase III Drosha initiates microRNA processing. Nature 425, 415-419.

Lee, Y., Kim, M., Han, J., Yeom, K.-H., Lee, S., Baek, S.H., and Kim, V.N. (2004). MicroRNA genes are transcribed by RNA polymerase II. EMBO J. 23, 4051-4060. 
Leenay, R.T., Maksimchuk, K.R., Slotkowski, R.A., Agrawal, R.N., Gomaa, A.A., Briner, A.E., Barrangou, R., and Beisel, C.L. (2016). Identifying and Visualizing Functional PAM Diversity across CRISPR-Cas Systems. Mol. Cell 62, 137-147.

Levinson, D.F., Duan, J., Oh, S., Wang, K., Sanders, A.R., Shi, J., Zhang, N., Mowry, B.J., Olincy, A., Amin, F., et al. (2011). Copy number variants in schizophrenia: confirmation of five previous findings and new evidence for $3 q 29$ microdeletions and VIPR2 duplications. Am. J. Psychiatry 168, 302-316.

Levitt, N., Briggs, D., Gil, A., and Proudfoot, N.J. (1989). Definition of an efficient synthetic poly(A) site. Genes Dev. 3, 1019-1025.

Li, B., Tadross, M.R., and Tsien, R.W. (2016). Sequential ionic and conformational signaling by calcium channels drives neuronal gene expression. Science 351, 863-867.

Li, L., Lin, X., Khvorova, A., Fesik, S.W., and Shen, Y. (2007). Defining the optimal parameters for hairpin-based knockdown constructs. RNA N. Y. N 13, 1765-1774.

Liu, B., Xu, H., Paton, J.F.R., and Kasparov, S. (2010). Cell- and region-specific miR30based gene knock-down with temporal control in the rat brain. BMC Mol. Biol. 11, 93.

Llinás, R., Steinberg, I.Z., and Walton, K. (1976). Presynaptic calcium currents and their relation to synaptic transmission: voltage clamp study in squid giant synapse and theoretical model for the calcium gate. Proc. Natl. Acad. Sci. U. S. A. 73, 2918-2922.

Love, M.I., Huber, W., and Anders, S. (2014). Moderated estimation of fold change and dispersion for RNA-seq data with DESeq2. Genome Biol. 15, 550.

Luo, J., Emanuele, M.J., Li, D., Creighton, C.J., Schlabach, M.R., Westbrook, T.F., Wong, K.-K., and Elledge, S.J. (2009). A Genome-wide RNAi Screen Identifies Multiple Synthetic Lethal Interactions with the Ras Oncogene. Cell 137, 835-848.

Lüscher, C., and Malenka, R.C. (2012). NMDA receptor-dependent long-term potentiation and long-term depression (LTP/LTD). Cold Spring Harb. Perspect. Biol. 4.

Ma, H., Groth, R.D., Cohen, S.M., Emery, J.F., Li, B., Hoedt, E., Zhang, G., Neubert, T.A., and Tsien, R.W. (2014). YCaMKII shuttles $\mathrm{Ca}^{2+} / \mathrm{CaM}$ to the nucleus to trigger CREB phosphorylation and gene expression. Cell 159, 281-294.

Ma, Q., Jones, D., and Springer, T.A. (1999). The chemokine receptor CXCR4 is required for the retention of $B$ lineage and granulocytic precursors within the bone marrow microenvironment. Immunity 10, 463-471.

Maier, R., Moser, G., Chen, G.-B., Ripke, S., Cross-Disorder Working Group of the Psychiatric Genomics Consortium, Coryell, W., Potash, J.B., Scheftner, W.A., Shi, J., Weissman, M.M., et al. (2015). Joint analysis of psychiatric disorders increases accuracy of risk prediction for schizophrenia, bipolar disorder, and major depressive disorder. Am. J. Hum. Genet. 96, 283-294.

Malenka, R.C., and Nicoll, R.A. (1993). NMDA-receptor-dependent synaptic plasticity: multiple forms and mechanisms. Trends Neurosci. 16, 521-527.

Malik, A.N., Vierbuchen, T., Hemberg, M., Rubin, A.A., Ling, E., Couch, C.H., Stroud, H., Spiegel, I., Farh, K.K.-H., Harmin, D.A., et al. (2014). Genome-wide identification and characterization of functional neuronal activity-dependent enhancers. Nat. Neurosci. 17, 1330-1339. 
Marín, O. (2012). Interneuron dysfunction in psychiatric disorders. Nat. Rev. Neurosci. 13, 107-120.

Martin, S.J., Grimwood, P.D., and Morris, R.G. (2000). Synaptic plasticity and memory: an evaluation of the hypothesis. Annu. Rev. Neurosci. 23, 649-711.

Maurano, M.T., Humbert, R., Rynes, E., Thurman, R.E., Haugen, E., Wang, H., Reynolds, A.P., Sandstrom, R., Qu, H., Brody, J., et al. (2012). Systematic localization of common disease-associated variation in regulatory DNA. Science 337, 1190-1195.

Mayer, M.L., Westbrook, G.L., and Guthrie, P.B. (1984). Voltage-dependent block by Mg2+ of NMDA responses in spinal cord neurones. Nature 309, 261-263.

Mayr, B., and Montminy, M. (2001). Transcriptional regulation by the phosphorylationdependent factor CREB. Nat. Rev. Mol. Cell Biol. 2, 599-609.

McBurney, R.N., and Neering, I.R. (1987). Neuronal calcium homeostasis. Trends Neurosci. 10, 164-169.

McClure, C., Cole, K.L.H., Wulff, P., Klugmann, M., and Murray, A.J. (2011). Production and titering of recombinant adeno-associated viral vectors. J. Vis. Exp. JoVE e3348.

McCown, T.J. (2005). Adeno-associated virus (AAV) vectors in the CNS. Curr. Gene Ther. 5, 333-338.

McLennan, H. (1981). Actions of the optical isomers of 2-amino-5-phosphonovalerate as antagonists of excitatory amino acids. Eur. J. Pharmacol. 73, 97-99.

Mellado, M., Rodríguez-Frade, J.M., Vila-Coro, A.J., Fernández, S., Martín de Ana, A., Jones, D.R., Torán, J.L., and Martínez-A, C. (2001). Chemokine receptor homo- or heterodimerization activates distinct signaling pathways. EMBO J. 20, 2497-2507.

Mellström, B., Naranjo, J.R., Foulkes, N.S., Lafarga, M., and Sassone-Corsi, P. (1993). Transcriptional response to CAMP in brain: specific distribution and induction of CREM antagonists. Neuron 10,655-665.

Melnikov, A., Murugan, A., Zhang, X., Tesileanu, T., Wang, L., Rogov, P., Feizi, S., Gnirke, A., Callan, C.G., Kinney, J.B., et al. (2012). Systematic dissection and optimization of inducible enhancers in human cells using a massively parallel reporter assay. Nat. Biotechnol. 30, 271-277.

Mertens, J., Wang, Q.-W., Kim, Y., Yu, D.X., Pham, S., Yang, B., Zheng, Y., Diffenderfer, K.E., Zhang, J., Soltani, S., et al. (2015). Differential responses to lithium in hyperexcitable neurons from patients with bipolar disorder. Nature 527, 95-99.

Mertens, J., Marchetto, M.C., Bardy, C., and Gage, F.H. (2016). Evaluating cell reprogramming, differentiation and conversion technologies in neuroscience. Nat. Rev. Neurosci. advance online publication.

Meves, H., and Pichon, Y. (1975). Proceedings: Effects of 4-aminopyridine on the potassium current in internally perfused giant axons of the squid. J. Physiol. 251,60P-62P.

Mohr, S.E., Smith, J.A., Shamu, C.E., Neumüller, R.A., and Perrimon, N. (2014). RNAi screening comes of age: improved techniques and complementary approaches. Nat. Rev. Mol. Cell Biol. 15, 591-600. 
Moiani, A., Paleari, Y., Sartori, D., Mezzadra, R., Miccio, A., Cattoglio, C., Cocchiarella, F., Lidonnici, M.R., Ferrari, G., and Mavilio, F. (2012). Lentiviral vector integration in the human genome induces alternative splicing and generates aberrant transcripts. J. Clin. Invest. 122, 1653-1666.

Montminy, M. (1997). Transcriptional regulation by cyclic AMP. Annu. Rev. Biochem. 66, 807-822.

Morgan, J.I., Cohen, D.R., Hempstead, J.L., and Curran, T. (1987). Mapping patterns of Cfos expression in the central nervous system after seizure. Science 237, 192-197.

Morgens, D.W., Deans, R.M., Li, A., and Bassik, M.C. (2016). Systematic comparison of CRISPR/Cas9 and RNAi screens for essential genes. Nat. Biotechnol.

Murphy, T.H., Worley, P.F., and Baraban, J.M. (1991). L-type voltage-sensitive calcium channels mediate synaptic activation of immediate early genes. Neuron 7, 625-635.

Nagappan, G., and Lu, B. (2005). Activity-dependent modulation of the BDNF receptor TrkB: mechanisms and implications. Trends Neurosci. 28, 464-471.

Nakai, H., Yant, S.R., Storm, T.A., Fuess, S., Meuse, L., and Kay, M.A. (2001). Extrachromosomal recombinant adeno-associated virus vector genomes are primarily responsible for stable liver transduction in vivo. J. Virol. 75, 6969-6976.

Nakamura, T., Barbara, J.G., Nakamura, K., and Ross, W.N. (1999). Synergistic release of $\mathrm{Ca} 2+$ from IP3-sensitive stores evoked by synaptic activation of mGluRs paired with backpropagating action potentials. Neuron 24, 727-737.

Narahashi, T., Anderson, N.C., and Moore, J.W. (1966). Tetrodotoxin does not block excitation from inside the nerve membrane. Science 153, 765-767.

Neves, S.R., Ram, P.T., and lyengar, R. (2002). G Protein Pathways. Science 296, $1636-$ 1639 .

Newell, K.A., and Matosin, N. (2014). Rethinking metabotropic glutamate receptor 5 pathological findings in psychiatric disorders: implications for the future of novel therapeutics. BMC Psychiatry 14, 23.

Ngo, V.N., Davis, R.E., Lamy, L., Yu, X., Zhao, H., Lenz, G., Lam, L.T., Dave, S., Yang, L., Powell, J., et al. (2006). A loss-of-function RNA interference screen for molecular targets in cancer. Nature 441, 106-110.

Nicholas, C.R., Chen, J., Tang, Y., Southwell, D.G., Chalmers, N., Vogt, D., Arnold, C.M., Chen, Y.-J.J., Stanley, E.G., Elefanty, A.G., et al. (2013). Functional Maturation of hPSCDerived Forebrain Interneurons Requires an Extended Timeline and Mimics Human Neural Development. Cell Stem Cell 12, 573-586.

Nicol, X., and Gaspar, P. (2014). Routes to cAMP: shaping neuronal connectivity with distinct adenylate cyclases. Eur. J. Neurosci. 39, 1742-1751.

Nieland, T.J.F., Logan, D.J., Saulnier, J., Lam, D., Johnson, C., Root, D.E., Carpenter, A.E., and Sabatini, B.L. (2014). High content image analysis identifies novel regulators of synaptogenesis in a high-throughput RNAi screen of primary neurons. PloS One 9, e91744.

Nowak, L., Bregestovski, P., Ascher, P., Herbet, A., and Prochiantz, A. (1984). Magnesium gates glutamate-activated channels in mouse central neurones. Nature 307, 462-465. 
Nugent, F.S., Penick, E.C., and Kauer, J.A. (2007). Opioids block long-term potentiation of inhibitory synapses. Nature 446, 1086-1090.

Nurnberger JI, Jr, Koller DL, Jung J, and et al (2014). Identification of pathways for bipolar disorder: A meta-analysis. JAMA Psychiatry 71, 657-664.

Ofengeim, D., Shi, P., Miao, B., Fan, J., Xia, X., Fan, Y., Lipinski, M.M., Hashimoto, T., Polydoro, M., Yuan, J., et al. (2012). Identification of small molecule inhibitors of neurite loss induced by $A \beta$ peptide using high content screening. J. Biol. Chem. 287, 8714-8723.

Ogata, H., Goto, S., Fujibuchi, W., and Kanehisa, M. (1998). Computation with the KEGG pathway database. Biosystems 47, 119-128.

Paddison, P.J., Silva, J.M., Conklin, D.S., Schlabach, M., Li, M., Aruleba, S., Balija, V., O'Shaughnessy, A., Gnoj, L., Scobie, K., et al. (2004). A resource for large-scale RNAinterference-based screens in mammals. Nature 428, 427-431.

Papassotiropoulos, A., and de Quervain, D.J.F. (2015). Failed drug discovery in psychiatry: time for human genome-guided solutions. Trends Cogn. Sci. 19, 183-187.

Park, H., and Poo, M. (2013). Neurotrophin regulation of neural circuit development and function. Nat. Rev. Neurosci. 14, 7-23.

Parnas, O., Jovanovic, M., Eisenhaure, T.M., Herbst, R.H., Dixit, A., Ye, C.J., Przybylski, D., Platt, R.J., Tirosh, I., Sanjana, N.E., et al. (2015). A Genome-wide CRISPR Screen in Primary Immune Cells to Dissect Regulatory Networks. Cell 162, 675-686.

Paulsen, B. da S., da Silveira, M.S., Galina, A., and Rehen, S.K. (2013). Pluripotent stem cells as a model to study oxygen metabolism in neurogenesis and neurodevelopmental disorders. Arch. Biochem. Biophys. 534, 3-10.

Penzes, P., Cahill, M.E., Jones, K.A., VanLeeuwen, J.-E., and Woolfrey, K.M. (2011). Dendritic spine pathology in neuropsychiatric disorders. Nat Neurosci 14, 285-293.

Pfenning, A.R., Schwartz, R., and Barth, A.L. (2007). A comparative genomics approach to identifying the plasticity transcriptome. BMC Neurosci. 8, 20.

Plath, N., Ohana, O., Dammermann, B., Errington, M.L., Schmitz, D., Gross, C., Mao, X., Engelsberg, A., Mahlke, C., Welzl, H., et al. (2006). Arc/Arg3.1 is essential for the consolidation of synaptic plasticity and memories. Neuron 52, 437-444.

Pratt, J., Winchester, C., Dawson, N., and Morris, B. (2012). Advancing schizophrenia drug discovery: optimizing rodent models to bridge the translational gap. Nat. Rev. Drug Discov. $11,560-579$.

Purcell, S.M., Moran, J.L., Fromer, M., Ruderfer, D., Solovieff, N., Roussos, P., O'Dushlaine, C., Chambert, K., Bergen, S.E., Kähler, A., et al. (2014). A polygenic burden of rare disruptive mutations in schizophrenia. Nature 506, 185-190.

Qi, L.S., Larson, M.H., Gilbert, L.A., Doudna, J.A., Weissman, J.S., Arkin, A.P., and Lim, W.A. (2013). Repurposing CRISPR as an RNA-guided platform for sequence-specific control of gene expression. Cell 152, 1173-1183.

Radio, N.M. (2012). Neurite outgrowth assessment using high content analysis methodology. Methods Mol. Biol. Clifton NJ 846, 247-260. 
Ragozzino, D., Renzi, M., Giovannelli, A., and Eusebi, F. (2002). Stimulation of chemokine CXC receptor 4 induces synaptic depression of evoked parallel fibers inputs onto Purkinje neurons in mouse cerebellum. J. Neuroimmunol. 127, 30-36.

Rajewsky, N. (2006). microRNA target predictions in animals. Nat. Genet. 38 Suppl, S8-13.

Redmond, L. (2008). Translating neuronal activity into dendrite elaboration: signaling to the nucleus. Neurosignals 16, 194-208.

Ripke, S., O'Dushlaine, C., Chambert, K., Moran, J.L., Kähler, A.K., Akterin, S., Bergen, S.E., Collins, A.L., Crowley, J.J., Fromer, M., et al. (2013). Genome-wide association analysis identifies 13 new risk loci for schizophrenia. Nat. Genet. 45, 1150-1159.

Rodríguez-Tornos, F.M., San Aniceto, I., Cubelos, B., and Nieto, M. (2013). Enrichment of conserved synaptic activity-responsive element in neuronal genes predicts a coordinated response of MEF2, CREB and SRF. PloS One 8, e53848.

Rossi, D., and Zlotnik, A. (2000). The biology of chemokines and their receptors. Annu. Rev. Immunol. 18, 217-242.

Rubinson, D.A., Dillon, C.P., Kwiatkowski, A.V., Sievers, C., Yang, L., Kopinja, J., Rooney, D.L., Zhang, M., Ihrig, M.M., McManus, M.T., et al. (2003). A lentivirus-based system to functionally silence genes in primary mammalian cells, stem cells and transgenic mice by RNA interference. Nat. Genet. 33, 401-406.

Sabatini, B.L., Oertner, T.G., and Svoboda, K. (2002). The life cycle of $\mathrm{Ca}(2+)$ ions in dendritic spines. Neuron 33, 439-452.

Sada, N., Lee, S., Katsu, T., Otsuki, T., and Inoue, T. (2015). Epilepsy treatment. Targeting LDH enzymes with a stiripentol analog to treat epilepsy. Science 347, 1362-1367.

Sala, C., Futai, K., Yamamoto, K., Worley, P.F., Hayashi, Y., and Sheng, M. (2003). Inhibition of dendritic spine morphogenesis and synaptic transmission by activity-inducible protein Homer1a. J. Neurosci. Off. J. Soc. Neurosci. 23, 6327-6337.

Sarkar, A., Marchetto, M.C., and Gage, F.H. (2015). Synaptic activity: An emerging player in schizophrenia. Brain Res.

Saunders, J.A., Gandal, M.J., and Siegel, S.J. (2012). NMDA antagonists recreate signal-tonoise ratio and timing perturbations present in schizophrenia. Neurobiol. Dis. 46, 93-100.

Sawicka, K., and Zukin, R.S. (2012). Dysregulation of mTOR signaling in neuropsychiatric disorders: therapeutic implications. Neuropsychopharmacol. Off. Publ. Am. Coll. Neuropsychopharmacol. 37, 305-306.

Scannell, J.W., and Bosley, J. (2016). When Quality Beats Quantity: Decision Theory, Drug Discovery, and the Reproducibility Crisis. PloS One 11, e0147215.

Scannell, J.W., Blanckley, A., Boldon, H., and Warrington, B. (2012). Diagnosing the decline in pharmaceutical R\&D efficiency. Nat. Rev. Drug Discov. 11, 191-200.

Schena, M., Shalon, D., Davis, R.W., and Brown, P.O. (1995). Quantitative Monitoring of Gene Expression Patterns with a Complementary DNA Microarray. Science 270, 467-470.

Schenone, M., Dančík, V., Wagner, B.K., and Clemons, P.A. (2013). Target identification and mechanism of action in chemical biology and drug discovery. Nat. Chem. Biol. 9, 232240. 
Schilling, K., Luk, D., Morgan, J.I., and Curran, T. (1991). Regulation of a fos-lacZ fusion gene: a paradigm for quantitative analysis of stimulus-transcription coupling. Proc. Natl. Acad. Sci. U. S. A. 88, 5665-5669.

Schizophrenia Working Group of the Psychiatric Genomics Consortium (2014). Biological insights from 108 schizophrenia-associated genetic loci. Nature 511, 421-427.

Schlabach, M.R., Luo, J., Solimini, N.L., Hu, G., Xu, Q., Li, M.Z., Zhao, Z., Smogorzewska, A., Sowa, M.E., Ang, X.L., et al. (2008). Cancer proliferation gene discovery through functional genomics. Science 319, 620-624.

Schmittgen, T.D., and Livak, K.J. (2008). Analyzing real-time PCR data by the comparative CT method. Nat. Protoc. 3, 1101-1108.

Schulte, J., Sepp, K.J., Wu, C., Hong, P., and Littleton, J.T. (2011). High-content chemical and RNAi screens for suppressors of neurotoxicity in a Huntington's disease model. PloS One 6, e23841.

Schwarz, D.S., Hutvágner, G., Du, T., Xu, Z., Aronin, N., and Zamore, P.D. (2003). Asymmetry in the assembly of the RNAi enzyme complex. Cell 115, 199-208.

Segal, M. (2005). Dendritic spines and long-term plasticity. Nat. Rev. Neurosci. 6, 277-284.

Sekar, A., Bialas, A.R., de Rivera, H., Davis, A., Hammond, T.R., Kamitaki, N., Tooley, K., Presumey, J., Baum, M., Van Doren, V., et al. (2016). Schizophrenia risk from complex variation of complement component 4 . Nature $530,177-183$.

Selemon, L.D., and Goldman-Rakic, P.S. (1999). The reduced neuropil hypothesis: a circuit based model of schizophrenia. Biol. Psychiatry 45, 17-25.

Shalem, O., Sanjana, N.E., Hartenian, E., Shi, X., Scott, D.A., Mikkelsen, T.S., Heckl, D., Ebert, B.L., Root, D.E., Doench, J.G., et al. (2014). Genome-scale CRISPR-Cas9 knockout screening in human cells. Science $343,84-87$.

Shalem, O., Sanjana, N.E., and Zhang, F. (2015). High-throughput functional genomics using CRISPR-Cas9. Nat. Rev. Genet. 16, 299-311.

Shalin, S.C., Hernandez, C.M., Dougherty, M.K., Morrison, D.K., and Sweatt, J.D. (2006). Kinase suppressor of Ras1 compartmentalizes hippocampal signal transduction and subserves synaptic plasticity and memory formation. Neuron 50, 765-779.

Sharma, K., Schmitt, S., Bergner, C.G., Tyanova, S., Kannaiyan, N., Manrique-Hoyos, N., Kongi, K., Cantuti, L., Hanisch, U.-K., Philips, M.-A., et al. (2015). Cell type- and brain region-resolved mouse brain proteome. Nat. Neurosci. 18, 1819-1831.

Sheng, M., and Greenberg, M.E. (1990). The regulation and function of c-fos and other immediate early genes in the nervous system. Neuron 4, 477-485.

Shepherd, J.D., Rumbaugh, G., Wu, J., Chowdhury, S., Plath, N., Kuhl, D., Huganir, R.L., and Worley, P.F. (2006). Arc/Arg3.1 mediates homeostatic synaptic scaling of AMPA receptors. Neuron $52,475-484$.

Sherrington, R., Rogaev, E.I., Liang, Y., Rogaeva, E.A., Levesque, G., Ikeda, M., Chi, H., Lin, C., Li, G., Holman, K., et al. (1995). Cloning of a gene bearing missense mutations in early-onset familial Alzheimer's disease. Nature 375, 754-760. 
Silva, J.M., Marran, K., Parker, J.S., Silva, J., Golding, M., Schlabach, M.R., Elledge, S.J., Hannon, G.J., and Chang, K. (2008). Profiling essential genes in human mammary cells by multiplex RNAi screening. Science 319, 617-620.

Sindreu, C.B., Scheiner, Z.S., and Storm, D.R. (2007). Ca2+ -stimulated adenylyl cyclases regulate ERK-dependent activation of MSK1 during fear conditioning. Neuron 53, 79-89.

Sohal, V.S., Zhang, F., Yizhar, O., and Deisseroth, K. (2009). Parvalbumin neurons and gamma rhythms enhance cortical circuit performance. Nature 459, 698-702.

Song, J., Bergen, S.E., Kuja-Halkola, R., Larsson, H., Landén, M., and Lichtenstein, P. (2015). Bipolar disorder and its relation to major psychiatric disorders: a family-based study in the Swedish population. Bipolar Disord. 17, 184-193.

Spiegel, I., Mardinly, A.R., Gabel, H.W., Bazinet, J.E., Couch, C.H., Tzeng, C.P., Harmin, D.A., and Greenberg, M.E. (2014). Npas4 regulates excitatory-inhibitory balance within neural circuits through cell-type-specific gene programs. Cell 157, 1216-1229.

Sprenger, J., Lynn Fink, J., Karunaratne, S., Hanson, K., Hamilton, N.A., and Teasdale, R.D. (2008). LOCATE: a mammalian protein subcellular localization database. Nucleic Acids Res. 36, D230-D233.

St Clair, D., Blackwood, D., Muir, W., Carothers, A., Walker, M., Spowart, G., Gosden, C., and Evans, H.J. (1990). Association within a family of a balanced autosomal translocation with major mental illness. Lancet Lond. Engl. 336, 13-16.

Steen, R.G., Mull, C., McClure, R., Hamer, R.M., and Lieberman, J.A. (2006). Brain volume in first-episode schizophrenia: systematic review and meta-analysis of magnetic resonance imaging studies. Br. J. Psychiatry J. Ment. Sci. 188, 510-518.

Stefánsson, H., Thorgeirsson, T.E., Gulcher, J.R., and Stefánsson, K. (2003). Neuregulin 1 in schizophrenia: out of Iceland. Mol. Psychiatry 8, 639-640.

Stefansson, H., Ophoff, R.A., Steinberg, S., Andreassen, O.A., Cichon, S., Rujescu, D., Werge, T., Pietiläinen, O.P.H., Mors, O., Mortensen, P.B., et al. (2009). Common variants conferring risk of schizophrenia. Nature $460,744-747$.

von Stein, A., and Sarnthein, J. (2000). Different frequencies for different scales of cortical integration: from local gamma to long range alpha/theta synchronization. Int. J. Psychophysiol. Off. J. Int. Organ. Psychophysiol. 38, 301-313.

Stephan, A.H., Barres, B.A., and Stevens, B. (2012). The complement system: an unexpected role in synaptic pruning during development and disease. Annu. Rev. Neurosci. 35, 369-389.

Stewart, S.A., DYKXHOORN, D.M., PALLISER, D., MIZUNO, H., YU, E.Y., AN, D.S., SABATINI, D.M., CHEN, I.S.Y., HAHN, W.C., SHARP, P.A., et al. (2003). Lentivirusdelivered stable gene silencing by RNAi in primary cells. RNA 9, 493-501.

Stuart, G., Spruston, N., Sakmann, B., and Häusser, M. (1997). Action potential initiation and backpropagation in neurons of the mammalian CNS. Trends Neurosci. 20, 125-131.

Sullivan, P.F., Kendler, K.S., and Neale, M.C. (2003). Schizophrenia as a complex trait: evidence from a meta-analysis of twin studies. Arch. Gen. Psychiatry 60, 1187-1192. 
Sweet, R.A., Henteleff, R.A., Zhang, W., Sampson, A.R., and Lewis, D.A. (2009). Reduced dendritic spine density in auditory cortex of subjects with schizophrenia. Neuropsychopharmacol. Off. Publ. Am. Coll. Neuropsychopharmacol. 34, 374-389.

Swinney, D.C., and Anthony, J. (2011). How were new medicines discovered? Nat. Rev. Drug Discov. 10, 507-519.

Szekanecz, Z., and Koch, A.E. (2001). Chemokines and angiogenesis. Curr. Opin. Rheumatol. 13, 202-208.

Takahashi, K., and Yamanaka, S. (2006). Induction of pluripotent stem cells from mouse embryonic and adult fibroblast cultures by defined factors. Cell 126, 663-676.

Tanenbaum, M.E., Gilbert, L.A., Qi, L.S., Weissman, J.S., and Vale, R.D. (2014). A protein tagging system for signal amplification in gene expression and fluorescence imaging. Cell $159,635-646$.

Tang, B., Wang, T., Wan, H., Han, L., Qin, X., Zhang, Y., Wang, J., Yu, C., Berton, F., Francesconi, W., et al. (2015). Fmr1 deficiency promotes age-dependent alterations in the cortical synaptic proteome. Proc. Natl. Acad. Sci. U. S. A. 112, E4697-4706.

Tennessen, J.A., Bigham, A.W., O'Connor, T.D., Fu, W., Kenny, E.E., Gravel, S., McGee, S., Do, R., Liu, X., Jun, G., et al. (2012). Evolution and functional impact of rare coding variation from deep sequencing of human exomes. Science 337, 64-69.

Thomson, A.M. (2000). Facilitation, augmentation and potentiation at central synapses. Trends Neurosci. 23, 305-312.

Tian, J., Ma, K., and Saaem, I. (2009). Advancing high-throughput gene synthesis technology. Mol. Biosyst. 5, 714-722.

Topol, A., Zhu, S., Tran, N., Simone, A., Fang, G., and Brennand, K.J. (2015). Altered WNT Signaling in Human Induced Pluripotent Stem Cell Neural Progenitor Cells Derived from Four Schizophrenia Patients. Biol. Psychiatry 78, e29-34.

Tran, P.B., and Miller, R.J. (2003). Chemokine receptors: signposts to brain development and disease. Nat. Rev. Neurosci. 4, 444-455.

Ubogu, E.E., Cossoy, M.B., and Ransohoff, R.M. (2006). The expression and function of chemokines involved in CNS inflammation. Trends Pharmacol. Sci. 27, 48-55.

Uhlhaas, P.J., and Singer, W. (2012). Neuronal dynamics and neuropsychiatric disorders: toward a translational paradigm for dysfunctional large-scale networks. Neuron 75, 963-980.

Vacic, V., McCarthy, S., Malhotra, D., Murray, F., Chou, H.-H., Peoples, A., Makarov, V., Yoon, S., Bhandari, A., Corominas, R., et al. (2011). Duplications of the neuropeptide receptor gene VIPR2 confer significant risk for schizophrenia. Nature 471, 499-503.

Varela, F., Lachaux, J.P., Rodriguez, E., and Martinerie, J. (2001). The brainweb: phase synchronization and large-scale integration. Nat. Rev. Neurosci. 2, 229-239.

Verkhratsky, A. (2004). Endoplasmic reticulum calcium signaling in nerve cells. Biol. Res. 37, 693-699.

Vincent, F., Loria, P., Pregel, M., Stanton, R., Kitching, L., Nocka, K., Doyonnas, R., Steppan, C., Gilbert, A., Schroeter, T., et al. (2015). Developing predictive assays: the phenotypic screening "rule of 3." Sci. Transl. Med. 7, 293ps15. 
Wagner, B.K., and Schreiber, S.L. (2016). The Power of Sophisticated Phenotypic Screening and Modern Mechanism-of-Action Methods. Cell Chem. Biol. 23, 3-9.

Wang, H., and Storm, D.R. (2003). Calmodulin-regulated adenylyl cyclases: cross-talk and plasticity in the central nervous system. Mol. Pharmacol. 63, 463-468.

Wang, X.J., and Buzsáki, G. (1996). Gamma oscillation by synaptic inhibition in a hippocampal interneuronal network model. J. Neurosci. Off. J. Soc. Neurosci. 16, 64026413.

Wang, T., Wei, J.J., Sabatini, D.M., and Lander, E.S. (2014). Genetic screens in human cells using the CRISPR-Cas9 system. Science 343, 80-84.

Watanabe, S., Hong, M., Lasser-Ross, N., and Ross, W.N. (2006). Modulation of calcium wave propagation in the dendrites and to the soma of rat hippocampal pyramidal neurons. J. Physiol. 575, 455-468.

Waters, J., Schaefer, A., and Sakmann, B. (2005). Backpropagating action potentials in neurones: measurement, mechanisms and potential functions. Prog. Biophys. Mol. Biol. 87, $145-170$.

Wen, Z., Nguyen, H.N., Guo, Z., Lalli, M.A., Wang, X., Su, Y., Kim, N.-S., Yoon, K.-J., Shin, J., Zhang, C., et al. (2014). Synaptic dysregulation in a human iPS cell model of mental disorders. Nature 515, 414-418.

West, A.E., and Greenberg, M.E. (2011). Neuronal activity-regulated gene transcription in synapse development and cognitive function. Cold Spring Harb. Perspect. Biol. 3.

West, A.E., Griffith, E.C., and Greenberg, M.E. (2002). Regulation of transcription factors by neuronal activity. Nat. Rev. Neurosci. 3, 921-931.

Whittington, M.A., Traub, R.D., and Jefferys, J.G. (1995). Synchronized oscillations in interneuron networks driven by metabotropic glutamate receptor activation. Nature 373 , 612-615.

Wiegert, J.S., and Bading, H. (2011). Activity-dependent calcium signaling and ERK-MAP kinases in neurons: a link to structural plasticity of the nucleus and gene transcription regulation. Cell Calcium 49, 296-305.

Wiegert, J.S., Bengtson, C.P., and Bading, H. (2007). Diffusion and not active transport underlies and limits ERK1/2 synapse-to-nucleus signaling in hippocampal neurons. J. Biol. Chem. 282, 29621-29633.

Williams, R.W., and Herrup, K. (1988). The control of neuron number. Annu. Rev. Neurosci. $11,423-453$.

Winkler, U., and Hirrlinger, J. (2015). Crosstalk of Signaling and Metabolism Mediated by the NAD(+)/NADH Redox State in Brain Cells. Neurochem. Res. 40, 2394-2401.

Winzeler, E.A., Shoemaker, D.D., Astromoff, A., Liang, H., Anderson, K., Andre, B., Bangham, R., Benito, R., Boeke, J.D., Bussey, H., et al. (1999). Functional Characterization of the S. cerevisiae Genome by Gene Deletion and Parallel Analysis. Science 285, 901-906.

Wray, N.R., Pergadia, M.L., Blackwood, D.H.R., Penninx, B.W.J.H., Gordon, S.D., Nyholt, D.R., Ripke, S., MacIntyre, D.J., McGhee, K.A., Maclean, A.W., et al. (2012). Genome-wide association study of major depressive disorder: new results, meta-analysis, and lessons learned. Mol. Psychiatry 17, 36-48. 
Xia, Y., Zhao, P., Xue, J., Gu, X.Q., Sun, X., Yao, H., and Haddad, G.G. (2003). Na+ channel expression and neuronal function in the $\mathrm{Na}+/ \mathrm{H}+$ exchanger 1 null mutant mouse. $\mathrm{J}$. Neurophysiol. 89, 229-236.

Xia, Z., Dudek, H., Miranti, C.K., and Greenberg, M.E. (1996). Calcium influx via the NMDA receptor induces immediate early gene transcription by a MAP kinase/ERK-dependent mechanism. J. Neurosci. Off. J. Soc. Neurosci. 16, 5425-5436.

Xing, J., Ginty, D.D., and Greenberg, M.E. (1996). Coupling of the RAS-MAPK pathway to gene activation by RSK2, a growth factor-regulated CREB kinase. Science 273, 959-963.

Yi, R., Qin, Y., Macara, I.G., and Cullen, B.R. (2003). Exportin-5 mediates the nuclear export of pre-microRNAs and short hairpin RNAs. Genes Dev. 17, 3011-3016.

Yizhar, O., Fenno, L.E., Prigge, M., Schneider, F., Davidson, T.J., O'Shea, D.J., Sohal, V.S., Goshen, I., Finkelstein, J., Paz, J.T., et al. (2011). Neocortical excitation/inhibition balance in information processing and social dysfunction. Nature 477, 171-178.

Yoshida, T., Imai, T., Kakizaki, M., Nishimura, M., Takagi, S., and Yoshie, O. (1998). Identification of single C motif-1/lymphotactin receptor XCR1. J. Biol. Chem. 273, 1655116554.

Yoshida, T., Izawa, D., Nakayama, T., Nakahara, K., Kakizaki, M., Imai, T., Suzuki, R., Miyasaka, M., and Yoshie, O. (1999). Molecular cloning of mXCR1, the murine SCM1/lymphotactin receptor. FEBS Lett. 458, 37-40.

Zeng, Y., and Cullen, B.R. (2004). Structural requirements for pre-microRNA binding and nuclear export by Exportin 5. Nucleic Acids Res. 32, 4776-4785.

Zhang, S.-J., Zou, M., Lu, L., Lau, D., Ditzel, D.A.W., Delucinge-Vivier, C., Aso, Y., Descombes, P., and Bading, H. (2009). Nuclear calcium signaling controls expression of a large gene pool: identification of a gene program for acquired neuroprotection induced by synaptic activity. PLoS Genet. 5, e1000604.

Zhu, J.J., Qin, Y., Zhao, M., Van Aelst, L., and Malinow, R. (2002). Ras and Rap control AMPA receptor trafficking during synaptic plasticity. Cell 110, 443-455.

Zilberter, Y. (2000). Dendritic release of glutamate suppresses synaptic inhibition of pyramidal neurons in rat neocortex. J. Physiol. 528, 489-496.

Zuber, J., McJunkin, K., Fellmann, C., Dow, L.E., Taylor, M.J., Hannon, G.J., and Lowe, S.W. (2011). Toolkit for evaluating genes required for proliferation and survival using tetracycline-regulated RNAi. Nat. Biotechnol. 29, 79-83.

Zucker, R.S., and Regehr, W.G. (2002). Short-term synaptic plasticity. Annu. Rev. Physiol. $64,355-405$. 


\section{Acknowledgement}

First of all, I want to thank Prof. Dr. Moritz Rossner for his supervision and his support over the last years. I am very grateful that I got the opportunity to do cutting-edge science and develop under his supervision into a passionate scientist with a strong interest in assay development.

I want to highlight the exceptional team spirit of the entire department for Molecular Neurobiology. This has been very important for the success of this project. In particular, I would like to give credit to Dr. Sven Wichert, Dr, Michael Wehr, Dr. Ben Brankatschk, and Nirmal Raman Kannaiyan, as they were always open for help and invaluable feedback, as well as great times outside the lab.

For proof-reading my thesis, I would in particular like to thank Dr. Michael Wehr and Dr. Ben Brankatschk.

Since many people have been involved in this project, I would like to extend my thanks to Beate Kauschat for her great assistance with the primary neuron cultures and to Dr. Sabrina Galinski, Stefanie Behrens, and Karin Neumeier for running the NGS facility.

I am grateful to my thesis committee members, Prof. Dr. Nils Brose and Dr. Camin Dean for the input during the committee meetings. 


\section{Curriculum vitae}

Personal data

$\begin{array}{ll}\text { Name } & \text { Alexander Herholt } \\ \text { Date of birth } & 16.05 .1986 \\ \text { Place of birth } & \text { Bielefeld } \\ \text { Nationality } & \text { German }\end{array}$

Education

2012 - current

\section{Graduate student}

GGNB Graduate School "Molecular Physiology of the Brain" Molecular Neurobiology, Department of Psychiatry, Ludwig-Maximilians-University Munich 2012-2014 Max-Planck-Institute of Experimental Medicine Göttingen, Department of Neurogenetics, Reseach group Gene expression and signaling

$2011-2012$

\section{Research associate}

Max-Planck-Institute of Experimental Medicine Göttingen, Department of Neurogenetics, Reseach group Gene expression and signaling

M. Sc. in Developmental, Neural, and Behavioral Biology

$2009-2011$

M.Sc. study in Developmental, Neural, and Behavioral Biology Georg-August University Göttingen

2009

\section{B.Sc. in Biology}

$2006-2009$

B.Sc. study in Biology

Georg-August University Göttingen

$2005-2006$

Social service

2005

Abitur

Martin-Niemöller Gesamtschule Bielefeld

\section{Publications}

Willig KI, Steffens H, Gregor C, Herholt A, Rossner MJ, Hell SW (2014)

Nanoscopy of filamentous actin in cortical dendrites of a living mouse.

Biophysical journal 106(1), L01-L03 